\title{
Empirical Decomposition of the IV-OLS Gap with Heterogeneous and Nonlinear Effects
}

\author{
Shoya Ishimaru*
}

June 27, 2022

\begin{abstract}
This study proposes an econometric framework to interpret and empirically decompose the difference between IV and OLS estimates given by a linear regression model when the true causal effects of the treatment are nonlinear in treatment levels and heterogeneous across covariates. I show that the IV-OLS coefficient gap consists of three estimable components: the difference in weights on the covariates, the difference in weights on the treatment levels, and the difference in identified marginal effects that arises from endogeneity bias. Applications of this framework to return-to-schooling estimates demonstrate the empirical relevance of this distinction in properly interpreting the IV-OLS gap.

JEL Classification: C21, C26, I26
\end{abstract}

\footnotetext{
*Hitotsubashi University, Department of Economics (email: shoya.ishimaru@r.hit-u.ac.jp). I am grateful to Mark Colas, Chao Fu, John Kennan, Lance Lochner, Nobuhiko Nakazawa, Masayuki Sawada, Tymon Słoczyński, Yoichi Sugita, Christopher Taber, Matthew Wiswall, and many seminar participants for their comments. I greatly appreciate helpful suggestions from the editor and anonymous referees. All errors are mine.
} 


\section{Introduction}

Instrumental variables (IV) regression is the most common approach for estimating the causal effect of a potentially endogenous regressor. A standard empirical approach specifies the following linear model:

$$
Y=\beta X+W^{\prime} \gamma+\varepsilon
$$

where $Y$ is the outcome of interest, $X$ is a scalar (multivalued) treatment, and $W$ is a vector of covariates. A standard econometric textbook takes the linear regression equation (11) as the true causal relationship and interprets the gap between the IV and ordinary least squares (OLS) coefficient estimates of $X$ as a consequence of endogeneity bias associated with omitted variables, selection, or measurement error.

If these interpretations fail to provide a plausible explanation for the IV-OLS coefficient gap, empirical researchers often consider the possibility that it instead arises because of how the IV and OLS coefficients place different weights on different treatment margins or groups of individuals. This interpretation treats the regression equation (11) as a linear projection model rather than a causal model, allowing for the treatment effects to be heterogeneous and nonlinear in the true causal relationship. For example, Card (1995a, 1999, 2001) suggests that the positive IV-OLS gaps in many return-to-schooling studies could be explained by higher returns among credit-constrained individuals, who are more likely to be affected by cost-related instruments. In addition, researchers sometimes perform an OLS regression restricted to a sample of likely complier groups or complying treatment margins to examine the robustness of the IV-OLS coefficient gap to the weight difference. As a prominent

example, Angrist and Krueger (1991) compute an OLS estimate of the return to schooling by restricting their sample to individuals with 9-12 years of schooling, who are expected to be most influenced by quarter-of-birth instruments. However, despite the widely recognized importance of the weight difference interpretation, relatively few studies attempt to formally quantify how much it actually matters for the IV-OLS coefficient gap.1

In this study, I propose an econometric framework to quantify the sources of the IV-OLS coefficient gap. I begin my analysis by considering the following causal model:

$$
Y=g(X, W)+U
$$

with a valid instrument $Z$ that is uncorrelated with an unobservable $U$ conditional on covariates $W$. With no restriction on the structural function $g$, the equation allows for the

\footnotetext{
${ }^{1}$ Notable exceptions include Kling (2001), Lochner and Moretti (2001), Mogstad and Wiswall (2010), Løken et al. (2012), and Lochner and Moretti (2015).
} 
treatment effects to be heterogeneous across covariates $W$ and nonlinear in treatment levels $X$ in any manner.2 I demonstrate that the OLS and IV estimates based on the linear regression equation (11), when the true model is (2), represent different weighted averages of the marginal effects of the treatment they identify.

Using the weighted-average representations, I then decompose the IV-OLS coefficient gap into three estimable components. These are: (i) the covariate weight difference, being the difference in how the IV and OLS coefficients place weights on the covariates $W$; (ii) the treatment-level weight difference, as the difference in how they place weights on treatment levels $X$; and (iii) the endogeneity bias (or marginal effect difference), being the difference between the IV- and OLS-identified marginal effects originating from the correlation between the treatment $X$ and the unobservable $U$. For instance, in the return-to-schooling context, (i) arises from heterogeneous returns across observed personal backgrounds with different responses to the instrument, which motivates the conjecture of Card (1995a, 1999, 2001), (ii) follows from nonlinear returns across schooling levels with different sensitivity to the instrument, which motivates the robustness check of Angrist and Krueger (1991), and (iii) corresponds to endogeneity bias associated with omitted unobserved ability.

To implement the decomposition, I propose a two-step approach to estimate the "IVweighted OLS" coefficients that serve as the intermediate points between the IV and OLS coefficients 3 The first step predicts the conditional mean of the outcome $Y$ given $(X, W)$, and the second step uses the prediction to construct a dependent variable and performs a quasiIV regression. Depending on the functional form restriction in the first step, the estimated OLS coefficients in the second step have the IV weights on the covariates or on both the covariates and treatment levels. Comparing the estimated IV-weighted OLS coefficients with the IV and OLS coefficients reveals the contributions of the weight difference components (i) and (ii) and the endogeneity bias component (iii). Standard statistical packages can compute the IV-weighted OLS estimates and perform statistical inference based on them 4

In an extension, I consider a class of identification strategies that use an instrument $Z$ deterministic in the covariates $W$, as in difference-in-differences (DID) and regression discontinuity $(\mathrm{RD})$ designs 5 I show that the weighted-average interpretation and the de-

\footnotetext{
${ }^{2}$ The separability restriction on the equation, which is relaxed in Section 3.2 rules out unobserved heterogeneity in the treatment effects.

${ }^{3}$ The concept of an IV-weighted OLS coefficient originates in Mogstad and Wiswall (2010), where they use the model $Y=g(X)+U$ and account for the treatment-level weight difference.

${ }^{4}$ The Stata package that implements the decomposition, ivolsdec, is available from the Boston College Statistical Software Components (SSC) archive. Type "ssc install ivolsdec" in the Stata command window to install it.

${ }^{5}$ Throughout this paper, the term "DID" refers to an identification strategy that exploits DID variation
} 
composition approach can also be applied to these setups, with only a small modification of the weight function.

It should be noted that my decomposition framework cannot fully isolate endogeneity bias in the presence of unobserved heterogeneity in the treatment effects. In an extension that relaxes the separability restriction on the equation (2), I demonstrate that the marginal effect difference component (iii) captures not only endogeneity bias but also the unobservabledriven discrepancy between the IV-identified and the average marginal effects 6 While the weight difference components (i) and (ii) remain informative about the implications of observed heterogeneity and nonlinearity, it can be misleading to attribute the marginal effect difference component (iii) entirely to endogeneity bias.

Using my framework, I examine the return-to-schooling estimates using several common IV strategies. The first example employs geographic variation in college costs (Cameron and Taber, 2004; Carneiro et al., 2011). The second exploits a discontinuity in the minimum school-leaving age across cohorts (Oreopoulos, 2006). The third and final example uses DID variation in compulsory schooling laws across cohorts and regions (Acemoglu and Angrist, 2000). In these empirical examples, the weight difference components are found to be as important as the endogeneity bias component in explaining the IV-OLS coefficient gap. The direction or extent of endogeneity bias implied by the estimated IV-OLS gap differs entirely by taking into consideration how the two coefficients place weights on the different observed personal backgrounds and schooling margins.

\section{Related Literature and Roadmap}

This paper advances the literature on the interpretation and decomposition of linear regression coefficients by exploring a general and empirically relevant setting in which the treatment effects are nonlinear in treatment levels and heterogeneous across covariates. The local average treatment effect (LATE) interpretation proposed by Imbens and Angrist (1994) in a binary treatment context originates the idea that the IV coefficient is a weighted average of the marginal causal effects of the treatment. Angrist and Imbens (1995) and Angrist et al. (2000) extend the basic insights of the LATE interpretation to a multivalued treatment case. Yitzhaki (1996) and Angrist and Krueger (1999) suggest the analogous weighted-average

in the instrument using a two-way fixed effects regression, in which the treatment and the instrument can be nonbinary. Examples include Acemoglu and Angrist (2000), Duflo (2001), Black et al. (2005), and a fuzzy DID setup considered in de Chaisemartin and D'Haultfoeuille (2018). The term "RD" refers to a fuzzy RD design, which is usually implemented as an IV regression.

${ }^{6}$ This corresponds to a classic impossibility result in the setting with a binary treatment and no covariates, where endogeneity bias cannot generally be separated from the difference between the local and population average treatment effects. 
interpretation of the OLS coefficient 7 Much of the focus of the literature has been on a univariate model with no or fixed covariates, which makes it difficult to immediately apply these results to empirical settings. While drawing on these existing results, my framework synthesizes them into an empirically relevant format and provides an estimable decomposition of the IV-OLS coefficient gap.

Motivated by the weighted-average interpretation developed in the literature, Mogstad and Wiswall (2010), Løken, Mogstad, and Wiswall (2012), and Lochner and Moretti (2015) propose the empirical decomposition of the IV-OLS coefficient gap into weight difference and endogeneity bias components. They consider a model in which the treatment effects are nonlinear in treatment levels but homogeneous across covariates. My framework generalizes these previous works by allowing the treatment effects to be heterogeneous across covariates. This is an empirically meaningful generalization, as my empirical applications demonstrate the relevance of the covariate weight difference in interpreting the IV-OLS coefficient gap.

While the linear IV regression is often advocated for its transparency (Angrist and Pischke, 2010), it is also criticized for its lack of a clear connection to an economic parameter of interest (Heckman and Urzua, 2010) 8 A related strand of the literature based on the latter view aims to develop alternatives to the linear IV regression, including the policyrelevant treatment effect proposed by Heckman and Vytlacil (2001) and Carneiro et al. (2010). Nevertheless, many empirical researchers use the linear regression for its simplicity. My framework aims to provide useful diagnostics for empirical researchers who use the linear regression while recognizing its potential limitations.

The rest of the paper proceeds as follows. Section 2 presents the interpretation of the linear IV and OLS coefficients and proposes the decomposition of the IV-OLS coefficient gap. Section 3 extends these econometric results by exploring settings with alternative assumptions. Section 4 proposes the estimators for the IV-weighted OLS coefficients for empirically performing the decomposition. Section 5 presents the results from the empirical applications, and Section 6 concludes. Online Appendix presents the proofs of the theorems, explores additional econometric results, and provides details for the empirical applications.

\footnotetext{
${ }^{7}$ The OLS interpretation is also explored by Angrist (1998), Aronow and Samii (2016), and Słoczyński $(2020)$ in a binary treatment case with a focus on observed heterogeneity.

${ }^{8}$ For the linear OLS regression, Słoczyński (2020) shows that the OLS coefficient of a binary treatment with covariates generally does not represent the average treatment effect (ATE), the average treatment effect on treated (ATT), or untreated (ATU).
} 


\section{Econometric Framework}

\subsection{Setup and Assumptions}

I consider a random draw of $(Y, X, W, Z)$, where $Y$ is a scalar outcome variable, $X$ is a scalar treatment variable, $W$ is a vector of covariates, and $Z$ is a scalar instrument. Multiinstrument two-stage least squares can fit into this setup by regarding the projection of the treatment $X$ onto the instrument vector and covariates $W$ (i.e., the first-stage predicted value) as a synthetic scalar instrument 9

Throughout this paper, I assume the existence of the first and second moments of any random variable. I use $L_{w}(R)=w^{\prime} E\left(W W^{\prime}\right)^{-1} E(W R)$ to denote a linear projection of a random variable $R$ onto $W$ evaluated at $W=w$ (i.e., a predicted value from a linear regression of $R$ on $W) 10$ I define $\widetilde{R}=R-L_{W}(R)$ to be a residual from the linear projection. Let $m(x, w)=E[Y \mid X=x, W=w]$ be the conditional mean function of $Y$ given $(X, W)$.

To assess the impact of the treatment $X$ on the outcome $Y$, a standard approach specifies a linear regression model:

$$
Y=\beta X+W^{\prime} \gamma+\varepsilon, E(\varepsilon W)=0
$$

Additional moment conditions $E(\varepsilon Z)=0$ and $E(\varepsilon X)=0$, respectively pin down the linear IV coefficient $\beta_{I V}$ and OLS coefficient $\beta_{O L S}$ as

$$
\begin{aligned}
\beta_{I V} & =E(\widetilde{Y} \widetilde{Z}) / E(\widetilde{X} \widetilde{Z}), \\
\beta_{O L S} & =E(\widetilde{Y} \widetilde{X}) / E\left(\widetilde{X}^{2}\right) .
\end{aligned}
$$

Note that I treat the equation (3) merely as a statistical model to characterize $\beta_{I V}$ and $\beta_{O L S}$, which does not impose any assumption on the underlying causal relationship.

To consider the causal interpretation of the IV and OLS coefficients, I define $Y(x)$ to be the potential outcome associated with the treatment level $x$, which produces the observed outcome as $Y=Y(X)$. The derivative $Y^{\prime}(x)$ is the marginal effect of the treatment in a causal sense. I make the following assumptions.

Assumption S. (Separability) The potential outcome is given by $Y(x)=g(x, W)+U$, $E(U \mid W)=0$.

\footnotetext{
${ }^{9}$ If a regression of $X$ on a vector instrument $\left(Z_{1}, \ldots, Z_{M}\right)$ controlling for $W$ yields a first-stage coefficient $\left(\pi_{1}, \ldots, \pi_{M}\right)$, I regard $Z=\sum_{m=1}^{M} \pi_{m} Z_{m}$ as a synthetic scalar instrument. As my setting allows for negative IV weights, a partial monotonicity condition for each individual instrument $Z_{m}$ as in Mogstad et al. (2021) is not required as long as the treatment $X$ and the synthetic instrument $Z$ are correlated conditional on $W$.

${ }^{10}$ The covariate vector $W$ includes unity as one of its elements.
} 
Assumption C. (Continuous Treatment) The treatment $X$ is continuously distributed on support $(\underline{x}, \bar{x})$, with $-\infty \leq \underline{x}<\bar{x} \leq \infty$.

Assumption D. (Regularity Conditions on Derivatives) Let $V_{a}^{b}(f, w)=\int_{\min \{a, b\}}^{\max \{a, b\}}\left|\frac{\partial}{\partial x} f(x, w)\right| d x$ be the total variation of a function $f(x, w)$ differentiable in $x$ between points $a$ and $b$.

(i) $g(x, w)$ is differentiable in $x$ and $E\left[V_{x_{0}}^{X}(g, W)^{2}\right]<\infty$ for some $x_{0} \in(\underline{x}, \bar{x})$;

(ii) $m(x, w)$ is differentiable in $x$ and $E\left[V_{x_{0}}^{X}(m, W)^{2}\right]<\infty$ for some $x_{0} \in(\underline{x}, \bar{x})$.

Assumption IV. The instrument $Z$ satisfies the conditions:

(i) (Exogeneity) $E(U \widetilde{Z})=0$; (ii) (Relevance) $E(\widetilde{X} \widetilde{Z}) \neq 0$.

Assumption OLS. The treatment residual has positive variance, i.e., $E\left(\widetilde{X}^{2}\right)>0$.

Assumption L. (Linearity of Conditional Means)

(i) $E(Z \mid W)$ is linear in $W$; (ii) $E(X \mid W)$ is linear in $W$.

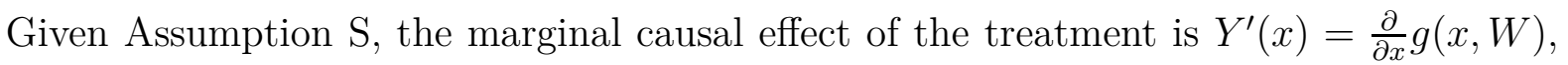
which can be nonlinear in treatment levels $x$ and heterogeneous across covariates $W$. However, it rules out unobserved heterogeneity in the effect 11 Section 3.2 relaxes this assumption.

I make Assumption C] to focus on a continuous treatment case, which is merely for expositional convenience 12 All econometric results can be applied to a discrete treatment case by extending $g(x, w)$ to nonsupport points of the treatment 13 Assumption D concerns the derivatives of the structural function $g$ and the conditional mean function $m$. Assumption IV is a set of standard IV assumptions that require the instrument to be exogenous and relevant after controlling for covariates. Assumption OLS is a standard OLS assumption.

Assumption $\mathrm{Q}$ is required for the exact weighted-average interpretation of the regression coefficients. A similar linearity assumption appears in, for example, Angrist and Krueger (1999), Lochner and Moretti (2015), and Słoczyński (2021). This assumption allows the analysis to abstract away from any omitted variable bias associated with unaccounted nonlinear effects of covariates, which is not as fundamental as endogeneity bias associated with unobservables. While this assumption mechanically holds in a saturated model in which a covariate vector $W$ consists of indicators for disjoint groups, it can be restrictive with continuous covariates. An empirical researcher may then want to choose elements of the vector

\footnotetext{
${ }^{11}$ The literature on the nonparametric IV approach typically makes a similar separability assumption. See, for example, Newey and Powell (2003), Blundell et al. (2007), and Horowitz (2011).

${ }^{12}$ As indicated in the assumption, the support $(\underline{x}, \bar{x})$ can be unbounded. Any integral expression of $x$ in this paper is taken over the support $(\underline{x}, \bar{x})$, which is kept implicit to simplify the exposition.

${ }^{13}$ For example, define $g(x, w)$ at nonsupport points by a linear interpolation without loss of generality. Then, the derivatives and integrals of $x$ in all econometric results can be replaced by the differences and summations of $x$.
} 
$W$ based on a series approximation and thereby flexibly account for the nonlinear and interaction effects of the underlying observables 14 Assumption $\mathrm{L}$ does not hold by construction for identification strategies based on DID or RD designs. Section 3.1 considers these cases.

\subsection{Weighted-Average Interpretation}

I start by presenting a key theorem for interpreting the IV and OLS coefficients. The OLS part of the theorem is shown by Angrist and Krueger (1999) in a discrete treatment setting. I present it for completeness and for reinterpretation in my setting.

Theorem 1. The IV and OLS coefficients have a weighted-average interpretation as below.

(i) With Assumptions $\underline{S}, \square, \square-(i), I V$, and $L-(i)$,

$$
\beta_{I V}=\iint \frac{\partial}{\partial x} g(x, w) \omega_{Z}(x, w) d F_{W}(w) d x
$$

(ii) (Angrist and Krueger, 1999) With Assumptions $\square$, D-(ii), OLS, and $L-(i i)$,

$$
\beta_{O L S}=\iint \frac{\partial}{\partial x} m(x, w) \omega_{X}(x, w) d F_{W}(w) d x
$$

The weight function is given by $\omega_{R}(x, w)=E\left[\widetilde{\mathbb{1}}_{X \geq x} \widetilde{R} \mid W=w\right] / E(\widetilde{X} \widetilde{R})$ for $R=Z$, X, which satisfies $\iint \omega_{R}(x, w) d F_{W}(w) d x=1$.

Theorem 1 implies that the IV and OLS coefficients are expressed as weighted averages of the marginal effects they identify. While the IV coefficient identifies a weighted average of the causal effects $\frac{\partial}{\partial x} g(x, w)$, the OLS coefficient identifies a weighted average of the slopes of the conditional mean function $\frac{\partial}{\partial x} m(x, w)$.

The relationship between the OLS-identified and IV-identified marginal effects, $\frac{\partial}{\partial x} m(x, w)$ and $\frac{\partial}{\partial x} g(x, w)$, can be expressed as

$$
\frac{\partial}{\partial x} m(x, w)=\frac{\partial}{\partial x} g(x, w)+\frac{\partial}{\partial x} E[U \mid X=x, W=w] .
$$

Therefore, the difference between the two marginal effects arises from endogeneity bias, i.e., the correlation between the treatment $X$ and the unobservable $U$.

While endogeneity bias makes the IV and OLS coefficients differ, an important implication from Theorem 1 is that the difference in the weight functions, $\omega_{Z}$ and $\omega_{X}$, also gives

\footnotetext{
${ }^{14}$ Appendix B.1 relaxes Assumption \to explore what happens when a good linear approximation is not feasible because of data limitations.
} 
rise to the IV-OLS coefficient gap. To explore what makes the IV weight $\omega_{Z}$ and the OLS weight $\omega_{X}$ differ, let $\bar{\omega}_{R}(w)=\int \omega_{R}(x, w) d x$ be the marginal weight on covariates $W=w$ for $R=Z, X$. The marginal IV and OLS weights on $W=w$ are given by:

$$
\begin{aligned}
& \bar{\omega}_{Z}(w)=\operatorname{Cov}(X, Z \mid W=w) / E(\widetilde{X} \widetilde{Z}), \\
& \bar{\omega}_{X}(w)=\operatorname{Var}(X \mid W=w) / E\left(\widetilde{X}^{2}\right) .
\end{aligned}
$$

The IV weight $\bar{\omega}_{Z}(w)$ is proportional to the conditional covariance $\operatorname{Cov}(X, Z \mid W=w)$, which is the product of the regression coefficient of $X$ on $Z$ given $W=w$ and the conditional variance $\operatorname{Var}(Z \mid W=w)$. This means that covariates $W$ with greater sensitivity of the treatment to the instrument or larger variation in the instrument are weighted more. In contrast, the OLS weight $\bar{\omega}_{X}(w)$ is proportional to the conditional variance $\operatorname{Var}(X \mid W=w)$. This implies that covariates $W$ with larger variation in the treatment are weighted more.

Similarly, let $\bar{\omega}_{R}(x)=\int \omega_{R}(x, w) d F_{W}(w)$ be the marginal weight on the treatment level $X=x$ for $R=Z, X$. The marginal IV and OLS weights on $X=x$ are given by:

$$
\begin{aligned}
& \bar{\omega}_{Z}(x)=E\left(\widetilde{\mathbb{1}}_{X \geq x} \widetilde{Z}\right) / E(\widetilde{X} \widetilde{Z}), \\
& \bar{\omega}_{X}(x)=E\left(\widetilde{\mathbb{1}}_{X \geq x} \widetilde{X}\right) / E\left(\widetilde{X}^{2}\right) .
\end{aligned}
$$

The IV weight $\bar{\omega}_{Z}(x)$ is proportional to a regression coefficient of $\mathbb{1}_{X \geq x}$ on the instrument $Z$, controlling for $W$. This implies that the more the instrument $Z$ influences the treatment $X$ at $x$, the more weighted the treatment level $x$ is. For example, if a compulsory schooling instrument $Z$ increases years of schooling $X$ through primary and secondary education but does not influence college education, the IV weight is expected to be positive with $x \leq 12$ and zero with $x>1215$ In general, the IV weight $\bar{\omega}_{Z}(x)$ is not guaranteed to be positive because the instrument can have positive effects on the treatment at some margins while having negative effects at others.

Interpreting the OLS weight expression is less straightforward, but Appendix C.1 shows

$$
\bar{\omega}_{X}(x) \propto \iiint\left(x_{1}-x_{2}\right) \mathbb{1}_{x_{2}<x \leq x_{1}} d F_{X \mid W}\left(x_{1} \mid w\right) d F_{X \mid W}\left(x_{2} \mid w\right) d F_{W}(w) .
$$

This implies that the OLS weight is proportional to the sum of differences between the pairs of conditionally independent observations $X_{1}, X_{2} \stackrel{i . i . d .}{\sim} F_{X \mid W}(\cdot \mid w)$ with $X_{1} \geq x>X_{2}$. Therefore, the treatment level $x$ is weighted more if the treatment $X$ is densely distributed both above and below $x$ As is evident from (11), the OLS weight $\bar{\omega}_{X}(x)$ on each treatment

\footnotetext{
${ }^{15}$ If $X$ is discrete, the weight on $x$ represents a change in treatment levels between $x-1$ and $x$.

${ }^{16}$ Yitzhaki (1996) derives the OLS weight function in a simpler case with no covariates and shows that
} 
level $x$ is nonnegative.

Theorem 1 can be considered as a generalization of Lochner and Moretti (2015), allowing for heterogeneity in the marginal effects across covariates. In fact, restricting $g(x, w)$ to be additively separable in $x$ and $w$ yields a weighted-average expression comparable to theirs. If $g(x, w)$ is additively separable, $\frac{\partial}{\partial x} g(x, w)$ depends only on $x$. This yields $\beta_{I V}=\int \frac{\partial}{\partial x} g(x, \cdot) \bar{\omega}_{Z}(x) d x$, which matches the weighted-average expression provided by Proposition 1 in Lochner and Moretti (2015).

\subsection{Related Work}

Theorem 1 closely relates to many existing results in the literature. To provide further intuition for the weighted-average interpretation, I explore the relationship between my results and those in the literature.

Denote the IV and OLS coefficients conditional on $W=w$ as

$$
\begin{aligned}
b_{I V}(w) & =\operatorname{Cov}(Y, Z \mid W=w) / \operatorname{Cov}(X, Z \mid W=w), \\
b_{O L S}(w) & =\operatorname{Cov}(Y, X \mid W=w) / \operatorname{Var}(X \mid W=w) .
\end{aligned}
$$

The following result from Lochner and Moretti (2015) shows that the IV and OLS coefficients, $\beta_{I V}$ and $\beta_{O L S}$, can be viewed as weighted averages of the covariate-specific coefficients, $b_{I V}(w)$ and $b_{O L S}(w)$.

Theorem 2. (Lochner and Moretti, 2015)

(i) With Assumptions IV-(ii) and L-(i),

$$
\beta_{I V}=\int b_{I V}(w) \bar{\omega}_{Z}(w) d F_{W}(w)
$$

(ii) With Assumptions OLS and $L-$-(ii),

$$
\beta_{O L S}=\int b_{O L S}(w) \bar{\omega}_{X}(w) d F_{W}(w)
$$

Note that this theorem does not rely on assumptions about the causal structure (Assumptions $\left[\mathrm{S}\right.$ and $[\mathrm{IV}-(\mathrm{i}))$ because $b_{I V}(w)$ is defined in (12) merely as a ratio of two conditional covariances. The IV and OLS weights on covariates $W=w$ match the marginal weights $\bar{\omega}_{Z}(w)$ and $\bar{\omega}_{X}(w)$ defined in (7) and (8) . This weighted-average interpretation is explored

the weight function is $\bar{\omega}_{X}(x) \propto(b-x)(x-a)$ if $X$ is uniformly distributed over $[a, b]$. The weights are zero at both ends of the support despite flat density because no pair of observations can sandwich the endpoints. 
intensively in a binary treatment case, in which the treatment effect is linear in treatment levels $x \in\{0,1\}$ by construction and it is possible to focus only on heterogeneity (Angrist, 1998; Aronow and Samii, 2016; Słoczyński, 2020, 2021).

The weighted-average interpretation of the covariate-specific coefficients, $b_{I V}(w)$ and $b_{O L S}(w)$, can be derived by applying Theorem 1 conditional on $W=w$. This result originates in Yitzhaki (1996) and Schechtman and Yitzhaki (2004).

Theorem 3. Let $\omega_{R}(x \mid w)=\omega_{R}(x, w) / \bar{\omega}_{R}(w)$ be the conditional weight on the treatment level $x$ given $W=w$.

(i) (Schechtman and Yitzhaki, 2004) With Assumptions $\underline{S}, \square, \square-(i), \operatorname{Cov}(U, Z \mid W=w)=0$, and $\operatorname{Cov}(X, Z \mid W=w) \neq 0$,

$$
b_{I V}(w)=\int \frac{\partial}{\partial x} g(x, w) \omega_{Z}(x \mid w) d x
$$

(ii) (Yitzhaki, 1996) With Assumptions $\square, \square-(i i)$, and $\operatorname{Var}(X \mid W=w)>0$,

$$
b_{O L S}(w)=\int \frac{\partial}{\partial x} m(x, w) \omega_{X}(x \mid w) d x
$$

The weighted-average expression for the covariate-specific IV coefficient $b_{I V}(w)$ could also be derived as a special case of the results from Angrist and Imbens (1995), Angrist et al. (2000), and Heckman et al. (2006), which consider a more general setting that allows for unobserved heterogeneity in the treatment effects 17

Synthesizing these existing results, Theorem 1 can be divided into two components: the linear IV and OLS coefficients, $\beta_{I V}$ and $\beta_{O L S}$, are weighted averages of the covariate-specific coefficients, $b_{I V}(w)$ and $b_{O L S}(w)$, with different weights on covariates (Theorem 2); and the covariate-specific coefficients are weighted averages of the identified marginal effects, $\frac{\partial}{\partial x} g(x, w)$ and $\frac{\partial}{\partial x} m(x, w)$, with different weights on treatment levels (Theorem 3 ).

\subsection{Decomposing the IV-OLS Coefficient Gap}

The weighted-average interpretation of the IV and OLS coefficients in Theorems 1 13 motivates the decomposition of the IV-OLS coefficient gap $\beta_{I V}-\beta_{O L S}$. I decompose the gap

\footnotetext{
${ }^{17}$ Angrist and Imbens (1995) allow for covariates in a special case with a "saturated" first stage, where $Z=E\left[X \mid Z_{1}, \ldots, Z_{M}, W\right]$ is generated from an instrument vector $\left(Z_{1}, \ldots, Z_{M}\right)$ and a covariate vector $W$ that both consist of indicators for disjoint groups.
} 
into the following three components.

$$
\begin{aligned}
\Delta_{C W} & =\int b_{O L S}(w)\left(\bar{\omega}_{Z}(w)-\bar{\omega}_{X}(w)\right) d F_{W}(w) \\
\Delta_{T W} & =\iint \frac{\partial}{\partial x} m(x, w)\left(\omega_{Z}(x \mid w)-\omega_{X}(x \mid w)\right) \bar{\omega}_{Z}(w) d F_{W}(w) d x \\
\Delta_{M E} & =\iint\left(\frac{\partial}{\partial x} g(x, w)-\frac{\partial}{\partial x} m(x, w)\right) \omega_{Z}(x, w) d F_{W}(w) d x .
\end{aligned}
$$

The first component, $\Delta_{C W}$, which I call "the covariate weight difference," corresponds to how differently the IV and OLS coefficients place weights on the covariates. The second component, $\Delta_{T W}$, which I call "the treatment-level weight difference," captures how differently the IV and OLS coefficients place weights on the treatment levels, conditional on the covariates. The third component, $\Delta_{M E}$, which I refer to as "the endogeneity bias" or "the marginal effect difference," captures the difference between the IV-and OLS-identified marginal effects, which arises from the endogeneity of the treatment as in (6). The sum of the three components is the IV-OLS gap, i.e., $\beta_{I V}-\beta_{O L S}=\Delta_{C W}+\Delta_{T W}+\Delta_{M E}$.

This decomposition departs from the OLS coefficient $\beta_{O L S}$ and arrives at the IV coefficient $\beta_{I V}$ by first changing the weights and then the marginal effects. This order follows an idea of the "IV-weighted OLS" approach adopted by Mogstad and Wiswall (2010) and Lochner and Moretti (2015). The advantage of this approach is that the decomposition is always feasible. The decomposition requires knowledge of the following IV-weighted OLS coefficients as intermediate points:

$$
\begin{aligned}
\beta_{C} & =\int b_{O L S}(w) \bar{\omega}_{Z}(w) d F_{W}(w) \\
\beta_{C T} & =\iint \frac{\partial}{\partial x} m(x, w) \omega_{Z}(x, w) d F_{W}(w) d x
\end{aligned}
$$

The first coefficient, $\beta_{C}$, is the OLS coefficient with the IV weight on covariates, while the second, $\beta_{C T}$, is the OLS coefficient with the IV weight on both covariates and treatment levels. By construction, $\Delta_{C W}=\beta_{C}-\beta_{O L S}, \Delta_{T W}=\beta_{C T}-\beta_{C}$, and $\Delta_{M E}=\beta_{I V}-\beta_{C T}$. These coefficients are always well-defined because $\omega_{Z}(x, w) \neq 0$ implies $\omega_{X}(x, w)>0$.

This is not a unique order in which the IV-OLS gap can be decomposed, as is the case with Blinder-Oaxaca type decomposition methods. For example, one can instead account for the marginal effect difference first, then the weight differences. However, this alternative order requires knowledge of the "OLS-weighted IV" coefficient, i.e., $\iint \frac{\partial}{\partial x} g(x, w) \omega_{X}(x, w) d F_{W}(x) d x$, as an intermediate point. This coefficient is not always identified because the instrument may 
have no variation or no impact on the treatment at some $(x, w)$, even with $\omega_{X}(x, w)>0.18$ This makes the OLS-weighted IV approach less practical despite its potential theoretical appeal 19

\section{Extensions}

I extend my econometric framework in several directions. Section 3.1 considers identification strategies based on DID and RD designs, in which the instrument $Z$ is deterministic in the covariates $W$. Section 3.2 relaxes Assumption $[S$ and allows for unobserved heterogeneity in the treatment effects. Appendix $\mathrm{B}$ explores additional extensions that consider a setting without Assumption $\mathrm{L}$, a setting with an invalid instrument, and a setting with DID or RD designs in the presence of unobserved heterogeneity.

\subsection{Identification Based on DID or RD Designs}

Assumption $\mathrm{L}$ does not hold by construction for two important identification strategies: DID and RD designs. I explore the weighted-average interpretation in these cases with an alternative set of assumptions.

With a DID-based identification strategy, each observation belongs to a particular group $g \in\{1, \ldots, G\}$ and period $t \in\{1, \ldots, T\}$, and the instrument $Z$ is constant within each $(g, t)$. The regression equation (3) can be written as

$$
Y=\beta X+\sum_{g=1}^{G} \gamma_{g} d_{g}+\sum_{t=1}^{T-1} \delta_{t} D_{t}+\varepsilon
$$

where $d_{g}$ indicates membership to a group $g$ and $D_{t}$ indicates membership to a period $t$. By construction, the instrument $Z$ is a deterministic and nonlinear function of the covariate vector $W=\left(d_{1}, \ldots, d_{G}, D_{1}, \ldots, D_{T-1}\right)$. This does not satisfy the requirement by Assumption L that $E(Z \mid W)$ should be linear in $W$. An empirical example from Acemoglu and Angrist (2000) in Section 5.3 fits into this setting.

With an RD-based identification strategy using a running variable $C$ with a cutoff $c$, the

\footnotetext{
${ }^{18} \mathrm{~L} \varnothing \mathrm{ken}$ et al. (2012) propose a mixture of the IV-weighted OLS and the OLS-weighted IV to make decomposition results independent of whether the marginal effect or the weight difference is accounted for first. This approach has the same identification issue as the OLS-weighted IV approach.

${ }^{19}$ In all empirical examples in Section 5 , the OLS-weighted IV coefficient cannot be identified because the instrument influences only a subset of treatment margins.
} 
instrument is $Z=\mathbb{1}_{C \geq c}$. The regression equation (3) can be written as

$$
Y=\beta X+\sum_{k=1}^{K} \gamma_{k} p_{k}(C)+\varepsilon
$$

where $\left(p_{1}, \ldots, p_{K}\right)$ is a set of basis functions with $p_{k}(c)=020$ The instrument $Z$ is a deterministic and nonlinear function of the covariate vector $W=\left(p_{1}(C), \ldots, p_{K}(C)\right)$. An empirical example from Oreopoulos (2006) in Section 5.2 fits into this setting.

The definition of the linear IV and OLS coefficients follows (41) and (5). I rule out a "sharp" DID or RD setup with $X=Z$, in which the IV and OLS coefficients are identical by construction. Instead of Assumption L, I make the following assumption to represent DIDand RD-based identification strategies.

Assumption LS. (Linear Structural Function) $g(x, w)$ is linear in $w$ for any $x \in(\underline{x}, \bar{x})$.

In a DID, this assumption corresponds to a parallel trend assumption, indicating that the group membership $d_{g}$ and the time membership $D_{t}$ additively affect a potential outcome. In an $\mathrm{RD}$, this assumption implies that the relationship between a potential outcome and a running variable $C$ is well approximated by a linear combination of the basis functions $\left(p_{1}(C), \ldots, p_{K}(C)\right)$ around $C=c$, which also implies continuity at $C=c$. In these settings, Assumption [V-(i) follows from $\operatorname{Var}(Z \mid W)=0$, as $E(U \widetilde{Z})=E(E(U \mid W) \widetilde{Z})=0$.

With Assumption $\mathrm{L}$ replaced by Assumption LS, the weighted-average interpretation of the IV coefficient remains valid with a modified weight function expression.

Theorem 4. With Assumptions $\left[S, \bar{C}, \bar{D}-(i), \underline{I V}\right.$, and $\underline{L S}$, the IV coefficient $\beta_{I V}$ is given by

$$
\beta_{I V}=\iint \frac{\partial}{\partial x} g(x, w) \omega_{Z}^{*}(x, w) d F_{W}(w) d x
$$

where $\omega_{Z}^{*}(x, w)=L_{w}\left(\mathbb{1}_{X \geq x} \widetilde{Z}\right) / E(\widetilde{X} \widetilde{Z})$ satisfies $\iint \omega_{Z}^{*}(x, w) d F_{W}(w) d x=1$.

Note that there are infinitely many weight functions other than $\omega_{Z}^{*}$ that can make this weighted-average expression. In particular, $\omega_{Z}^{*}(x, w)+h(x, w)-L_{w}(h(x, W))$ can also be a weight function, where $h(x, w)$ is any nonlinear function. This property is a mere artifact of Assumption LS, which makes the marginal effect $\frac{\partial}{\partial x} g(x, w)$ linear in $w$ and orthogonal to any linear projection residual. Therefore, it is most reasonable to use the linearly projected

\footnotetext{
${ }^{20}$ Given that an RD with local polynomials can be interpreted as a kernel-weighted version of an RD with global polynomials, my description focuses on a global polynomial case. The IV weight function should be multiplied by a kernel weight in a local polynomial case.
} 
function $\omega_{Z}^{*}$ and omit the redundant variation in weights 21

Given the weighted-average interpretation provided by Theorem 4 , it is possible to define the weight difference and the marginal effect difference components comparably to (14-16), using $\omega_{Z}^{*}$ instead of $\omega_{Z}$ as the IV weight function 22 Note that the marginal weights on covariate $W=w$ and treatment level $X=x$ are given by

$$
\begin{aligned}
& \bar{\omega}_{Z}^{*}(w)=\int \omega_{Z}^{*}(x, w) d x=L_{w}(X \widetilde{Z}) / E(X \widetilde{Z}), \\
& \bar{\omega}_{Z}^{*}(x)=\int \omega_{Z}^{*}(x, w) d F_{W}(w)=E\left(\mathbb{1}_{X \geq x} \widetilde{Z}\right) / E(X \widetilde{Z}) .
\end{aligned}
$$

While the treatment-level weight $\bar{\omega}_{Z}^{*}(x)$ is identical to $\bar{\omega}_{Z}(x)$ defined in (9), the covariate weight $\bar{\omega}_{Z}^{*}(w)$ has a different expression from $\bar{\omega}_{Z}(w)$ defined in (7).

\subsection{Unobserved Heterogeneity}

Assumption S rules out unobserved heterogeneity in the marginal effects of the treatment. This is a common but strong assumption. Because the distinction between observables and unobservables arises merely from data availability, it is natural to consider heterogeneity in both observed and unobserved dimensions in the econometric model. Allowing for unobserved heterogeneity in the marginal effects $Y^{\prime}(x)$, I define the average marginal effect (AME) as $\tau(x, w)=E\left[Y^{\prime}(x) \mid W=w\right]$. With Assumption $\underline{\mathrm{S}}$, the AME reduces to $\tau(x, w)=\frac{\partial}{\partial x} g(x, w)$.

Removing Assumptions S, D-(i), and IV-(i), I make the following set of assumptions about the potential outcome process $Y(x)$.

Assumption $\mathbf{P}$. The potential outcome process $Y(x)$ satisfies the following conditions:

(i) (Conditional Independence) $\operatorname{Cov}(Y(x), Z \mid W)=0$ for any $x \in(\underline{x}, \bar{x})$;

(ii) (Differentiability) $Y^{\prime}(x)$ exists for any $x \in(\underline{x}, \bar{x})$ and the total variation $V_{x_{0}}^{X}(Y(\cdot))=$ $\int_{\min \left\{x_{0}, X\right\}}^{\max \left\{x_{0}, X\right\}}\left|Y^{\prime}(x)\right| d x$ satisfies $E\left[V_{x_{0}}^{X}(Y(\cdot))^{2}\right]<\infty$ for some $x_{0} \in(\underline{x}, \bar{x})$.

Assumption $[\mathrm{P}$-(i) replaces $[\mathrm{IV}-(\mathrm{i})$, and this is a standard exogeneity assumption 23 As-

\footnotetext{
${ }^{21}$ de Chaisemartin and D'Haultfoeuille (2020) suggest the weight function expression for a DID with a binary treatment, which is an important special case of Theorem 4. Appendix C.2 discusses the relationship between Theorem 4 and their results.

${ }^{22}$ It still requires Assumption L-(ii) for the OLS coefficient to have an exact weighted-average interpretation. In this situation, a researcher may want to choose a more flexible specification in performing the OLS regression, e.g., controlling for group-time effects instead of additive group and time effects.

${ }^{23}$ This setting implicitly rules out any direct causal effects of the instrument on the potential outcome. Some studies explicitly specify the potential outcome $Y(x, z)$ to be a function of the potential instrument assignment $z$ and then assume $Y\left(x, z^{\prime}\right)=Y(x, z)$ for any $z \neq z^{\prime}$.
} 
sumption $\mathrm{P}$-(ii) replaces $\mathrm{D}$-(i). Unobserved heterogeneity in the treatment effects arises when $\operatorname{Var}\left(Y^{\prime}(x) \mid W=w\right)>0$. To rule out negative weights, some studies in the IV literature specify the potential treatment process and assume it to be monotonic in the instrument. On the other hand, I do not impose the monotonicity condition and maintain Assumption IV-(ii) (i.e., $E(\widetilde{X} \widetilde{Z}) \neq 0$ ), thereby allowing for negative weights 24

The following theorem extends the weighted-average interpretation of the IV coefficient provided by Theorem 1 .

Theorem 5. With Assumptions $\left[\mathrm{P}, \mathrm{C}, \overline{I V}-(i i)\right.$, and $\bar{L}-(i)$, the IV coefficient $\beta_{I V}$ is given by

$$
\beta_{I V}=\iint \tau_{I V}(x, w) \omega_{Z}(x, w) d F_{W}(w) d x
$$

where the IV-identified marginal effect $\tau_{I V}(x, w)$ at each $(x, w)$ is given by

$$
\tau_{I V}(x, w)=E\left[Y^{\prime}(x) \lambda\left(Y^{\prime}(x) \mid x, w\right) \mid W=w\right]
$$

with $\lambda(t \mid x, w)=\frac{\operatorname{Cov}\left(\mathbb{1}_{X \geq x}, Z \mid Y^{\prime}(x)=t, W=w\right)}{\operatorname{Cov}\left(\mathbb{1}_{X \geq x}, Z \mid W=w\right)}$ and $E\left[\lambda\left(Y^{\prime}(x) \mid x, w\right) \mid W=w\right]=1$.

Theorem [5implies that the IV coefficient is a weighted average of the causal effects $Y^{\prime}(x)$. Although treatment levels $x$ and covariates $w$ are weighted exactly in the same manner as in Theorem 1, unobserved heterogeneity influences how the effects $Y^{\prime}(x)$ are weighted. In particular, the difference between the IV-identified marginal effect $\tau_{I V}(x, w)$ and the AME $\tau(x, w)$ can be written as

$$
\tau_{I V}(x, w)-\tau(x, w)=\operatorname{Cov}\left(Y^{\prime}(x), \lambda\left(Y^{\prime}(x) \mid x, w\right) \mid W=w\right)
$$

This difference arises from unobservable-driven covariance between the treatment effects $Y^{\prime}(x)$ and the treatment responses to the instrument. For example, suppose some unobservables positively influence $Y^{\prime}(x)$ and make the treatment more responsive to the instrument. As the weight function $\lambda(t \mid x, w)$ represents how strongly the instrument $Z$ induces a transition of the treatment $X$ from below $x$ to above $x$ conditional on $Y^{\prime}(x)=t$ and $W=w$, $\tau_{I V}(x, w)>\tau(x, w)$ results from a greater emphasis on a higher $Y^{\prime}(x)$. This difference corresponds to the discrepancy between the LATE and ATE in a binary treatment setting with no covariates, although the difference is specific to each treatment level $x$ and covariate value $w$ in this case.

\footnotetext{
${ }^{24}$ Appendix C.3 considers the weighted-average interpretation under the monotonicity condition and explores its relationship with the LATE interpretation in Angrist and Imbens (1995) and the marginal treatment effect interpretation in Heckman et al. (2006).
} 
Given the weighted-average interpretation, the decomposition in (14 16) remains valid by replacing the marginal effect difference component in (16) with

$$
\Delta_{M E}=\iint\left(\tau_{I V}(x, w)-\frac{\partial}{\partial x} m(x, w)\right) \omega_{Z}(x, w) d F_{W}(w) d x
$$

However, this component no longer represents endogeneity bias alone. In particular, (19) can be further decomposed as

$$
\begin{aligned}
\Delta_{M E} & =\iint\left(\tau(x, w)-\frac{\partial}{\partial x} m(x, w)\right) \omega_{Z}(x, w) d F_{W}(w) d x \\
& +\iint\left(\tau_{I V}(x, w)-\tau(x, w)\right) \omega_{Z}(x, w) d F_{W}(w) d x
\end{aligned}
$$

The first term captures endogeneity bias through the difference between the AME $\tau(x, w)$ and the slope of the conditional mean function $\frac{\partial}{\partial x} m(x, w)$. The second term represents the unobservable-driven weight difference discussed above.

In a nonbinary treatment setting, it is well known that the $\operatorname{AME} \tau(x, w)$ or analogous causal objects cannot be identified without any restriction on unobserved heterogeneity or causal structure 25 Thus, endogeneity bias and the unobservable-driven weight difference cannot be identified separately in general. Nevertheless, it remains helpful to isolate the covariate and treatment-level weight difference components using the decomposition, rather than observing only the raw IV-OLS gap.

\section{Estimation and Inference}

\subsection{IV-Weighted OLS Estimators}

Performing the decomposition proposed in Section 2 requires estimators of the IV-weighted OLS coefficients defined in (17, 18), which serve as intermediate points between the IV and OLS estimates. To define the estimators, let $\left(Y_{i}, X_{i}, Z_{i}, W_{i}\right)_{i=1}^{N}$ be an i.i.d. random sample that satisfies the set of assumptions in Section 2, The most natural estimators for the

\footnotetext{
${ }^{25}$ Examples of restrictions that may enable this identification are: one-dimensional unobservable in the first-stage relationship (Imbens and Newey, 2009); one-dimensional unobservable in the potential outcome (Chernozhukov et al., 2007); and the linear random coefficients model (Masten and Torgovitsky, 2016). One could estimate one of these models and recover the AME to further decompose $\Delta_{M E}$ into endogeneity bias and the unobservable-driven weight difference. Exploring the decomposition under these restrictions is beyond the scope of this paper.
} 
IV-weighted OLS coefficients are their direct data counterparts:

$$
\begin{aligned}
\widehat{\beta}_{C} & =\frac{1}{N} \sum_{i=1}^{N} \int \widehat{b}_{O L S}\left(W_{i}\right) \widehat{\omega}_{Z}\left(x, W_{i}\right) d x, \\
\widehat{\beta}_{C T} & =\frac{1}{N} \sum_{i=1}^{N} \int \frac{\partial}{\partial x} \widehat{m}\left(x, W_{i}\right) \widehat{\omega}_{Z}\left(x, W_{i}\right) d x,
\end{aligned}
$$

using some estimators $\left(\widehat{b}_{O L S}, \widehat{m}, \widehat{\omega}_{Z}\right)$ for $\left(b_{O L S}, m, \omega_{Z}\right)$.

While there are various choices for these estimators $\left(\widehat{b}_{O L S}, \widehat{m}, \widehat{\omega}_{Z}\right)$, I focus on the most practical setting. I assume that $\widehat{m}$ is a consistent estimator for $m$ estimated by minimizing $\frac{1}{N} \sum_{i=1}^{N}\left\{Y_{i}-\widehat{m}\left(X_{i}, W_{i}\right)\right\}^{2}$, and that $\widehat{m}(x, w)$ is given in a series form

$$
\widehat{m}(x, w)=\sum_{k=1}^{K_{N}} \widehat{\alpha}_{k}(w) p_{k}(x), \quad \widehat{\alpha}_{k}(w)=\sum_{\ell=1}^{L_{N}^{(k)}} \widehat{\theta}_{k \ell} q_{k \ell}(w)
$$

where $\left\{p_{k}(x), q_{k 1}(w), \ldots, q_{k L_{N}^{(k)}}(w)\right\}_{k=1}^{K_{N}}$ is a set of basis functions chosen by the researcher. The numbers of the basis functions $K_{N}$ and $\left(L_{N}^{(k)}\right)_{k=1}^{K_{N}}$ are constant in a parametric approach, while they may increase with the sample size $N$ with a nonparametric sieve estimation adopted. While $\widehat{b}_{O L S}$ can be any consistent estimator for $b_{O L S}$, for concreteness I assume that $\widehat{b}_{O L S}$ is also estimated by the series form (22) with $K_{N}=2$ and $\left(p_{1}(x), p_{2}(x)\right)=(1, x)$, in which $\widehat{\alpha}_{2}(w)$ corresponds to $\widehat{b}_{O L S}(w)$. In addition, as the estimator for $\omega_{Z}\left(x, W_{i}\right)$, I consider its sample analogue

$$
\widehat{\omega}_{Z}\left(x, W_{i}\right)=\frac{\widetilde{\mathbb{1}}_{X_{i} \geq x} \widetilde{Z}_{i}}{\frac{1}{N} \sum_{j=1}^{N} \widetilde{\mathbb{1}}_{X_{j} \geq x} \widetilde{Z}_{j}} .
$$

Then, (20 21) can be rewritten as

$$
\begin{aligned}
\widehat{\beta}_{C} & =\frac{\sum_{i=1}^{N} \widehat{b}_{O L S}\left(W_{i}\right) \widetilde{X}_{i} \widetilde{Z}_{i}}{\sum_{i=1}^{N} \widetilde{X}_{i} \widetilde{Z}_{i}} \\
\widehat{\beta}_{C T} & =\frac{\sum_{i=1}^{N}\left(\sum_{k=1}^{K_{N}} \widehat{\alpha}_{k}\left(W_{i}\right) \widetilde{P}_{i k}\right) \widetilde{Z}_{i}}{\sum_{i=1}^{N} \widetilde{X}_{i} \widetilde{Z}_{i}}
\end{aligned}
$$

where $P_{i k}=p_{k}\left(X_{i}\right)$. Therefore, the following two-step procedure gives the IV-weighted OLS estimates.

Step 1: Estimate $\widehat{b}_{O L S}(w)$ and $\left\{\widehat{\alpha}_{k}(w)\right\}_{k=1}^{K_{N}}$ using the least-squares method with the series specification (22). 
Step 2: Regress $Y_{2 i}=\sum_{k=1}^{K_{N}} \widehat{\alpha}_{k}\left(W_{i}\right) \widetilde{P}_{i k}$ on $\left(X_{i}, W_{i}\right)$ instrumenting $X_{i}$ with $Z_{i}$, which yields $\widehat{\beta}_{C T}$ as the coefficient of $X_{i}$. To estimate $\widehat{\beta}_{C}$, let $Y_{2 i}=\widehat{b}_{O L S}\left(W_{i}\right) \widetilde{X}_{i}$ and perform the same regression.

Note that this two-step approach naturally generalizes the one proposed by Lochner and Moretti (2015) by allowing for a more general functional form of $\widehat{m}(x, w)$. In fact, $\widehat{\beta}_{C T}$ given in Step 2 is identical to the reweighted OLS estimator in Lochner and Moretti (2015) if $\widehat{m}(x, w)$ is specified in Step 1 to be additively separable in $x$ and $w$.

\section{DID- or RD-type Instrument Case}

The setting considered in Section 3.1 requires a small modification of the estimators due to a difference in the weight functions. Suppose that the weight function $\omega_{Z}^{*}\left(x, W_{i}\right)$ defined in Theorem 4 is estimated by its sample analogue

$$
\widehat{\omega}_{Z}^{*}\left(x, W_{i}\right)=\frac{\widehat{L}_{W_{i}}\left(\mathbb{1}_{X_{i} \geq x} \widetilde{Z}_{i}\right)}{\frac{1}{N} \sum_{j=1}^{N} \mathbb{1}_{X_{j} \geq x} \widetilde{Z}_{j}},
$$

where $\widehat{L}_{w}\left(R_{i}\right)=w^{\prime}\left(\sum_{j=1}^{N} W_{j} W_{j}^{\prime}\right)^{-1}\left(\sum_{j=1}^{N} W_{j} R_{j}\right)$ is the linear projection estimate. Using $\widehat{\omega}_{Z}^{*}$ instead of $\widehat{\omega}_{Z}$ in deriving (23) 24) yields

$$
\begin{aligned}
\widehat{\beta}_{C} & =\frac{\sum_{i=1}^{N} \widehat{L}_{W_{i}}\left(\widehat{b}_{O L S}\left(W_{i}\right)\right) X_{i} \widetilde{Z}_{i}}{\frac{1}{N} \sum_{i=1}^{N} \widetilde{X}_{i} \widetilde{Z}_{i}}, \\
\widehat{\beta}_{C T} & =\frac{\sum_{i=1}^{N}\left(\sum_{k=1}^{K_{N}} \widehat{L}_{W_{i}}\left(\widehat{\alpha}_{k}\left(W_{i}\right)\right) P_{i k}\right) \widetilde{Z}_{i}}{\frac{1}{N} \sum_{i=1}^{N} \widetilde{X}_{i} \widetilde{Z}_{i}} .
\end{aligned}
$$

These expressions differ from (23 24) in two ways. First, $\widehat{b}_{O L S}\left(W_{i}\right)$ and $\widehat{\alpha}_{k}\left(W_{i}\right)$ are linearly projected onto $W_{i}$. In practice, $\widehat{b}_{O L S}\left(W_{i}\right)$ and $\widehat{\alpha}_{k}\left(W_{i}\right)$ may be specified to be linear at the outset, rather than estimating them from a flexible nonlinear specification first and then linearly predicting them. Second, $X_{i}$ and $P_{i k}$ instead of $\widetilde{X}_{i}$ and $\widetilde{P}_{i k}$ enter the numerators. These differences slightly change Step 2 in estimating the IV-weighted OLS estimates as follows.

Step 2 (DID- or RD-type Instrument): Regress $Y_{2 i}=\sum_{k=1}^{K_{N}} \widehat{L}_{W_{i}}\left(\widehat{\alpha}_{k}\left(W_{i}\right)\right) P_{i k}$ on $\left(X_{i}, W_{i}\right)$ instrumenting $X_{i}$ with $Z_{i}$, which yields $\widehat{\beta}_{C T}$ as the coefficient of $X_{i}$. To estimate $\widehat{\beta}_{C}$, let $Y_{2 i}=\widehat{L}_{W_{i}}\left(\widehat{b}_{O L S}\left(W_{i}\right)\right) X_{i}$ and perform the same regression. 


\subsection{Asymptotic Properties}

Asymptotic properties of the IV-weighted OLS estimator can be derived using the standard econometric results for a two-step estimator. The following discussion focuses on the case in which the first step is parametric, since Ackerberg et al. (2012) illustrate that a semiparametric two-step estimator that uses a series approximation in the first step can be treated as if it were a parametric estimator for the purpose of standard error computation.

Using the standard formula for a parametric two-step estimator (Newey and McFadden, 1994, Section 6), Appendix C.4 derives the asymptotic equivalence:

$$
\sqrt{N}\left(\widehat{\beta}_{C T}-\beta_{C T}\right) \underset{p}{\rightarrow} \frac{\frac{1}{\sqrt{N}} \sum_{i=1}^{N}\left(v_{1 i} \widehat{\widetilde{Z}}_{i}+v_{2 i} \widetilde{Z}_{i}\right)}{E\left[\widetilde{X}_{i} \widetilde{Z}_{i}\right]},
$$

where $v_{1 i}=Y_{i}-\sum_{k=1}^{K} \alpha_{k}\left(W_{i}\right) P_{i k}$ is a residual from the first step, $v_{2 i}=\widetilde{Y}_{2 i}-\beta_{C T} \tilde{X}_{i}$ is a residual from the second step, and $\widehat{\widetilde{Z}}_{i}$ is a predicted value of $\widetilde{Z}_{i}$ given by the first step using $\widetilde{Z}_{i}$ instead of $Y_{i}$ as the dependent variable 26 Most statistical packages can estimate the standard error of $\widehat{\beta}_{C T}$ by estimating the standard error of the right-hand side of (27) under a certain distributional assumption (heteroscedasticity-robust, clustered, etc.). For example, in an i.i.d. heteroscedastic case, the asymptotic variance of $\sqrt{N}\left(\widehat{\beta}_{C T}-\beta_{C T}\right)$ is given by $V_{\beta_{C T}}=E\left[\widetilde{X}_{i} \widetilde{Z}_{i}\right]^{-1} E\left[\left(v_{1 i} \widehat{\widetilde{Z}}_{i}+v_{2 i} \widetilde{Z}_{i}\right)^{2}\right]$ and the standard error of $\widehat{\beta}_{C T}$ can be estimated using the sample analogue of $\sqrt{V_{\beta_{C T}} / N}$. Estimating the standard error of $\widehat{\beta}_{C}$ can follow the same procedure, as it is a special case with $K=2$.

\subsection{Testing the Treatment Endogeneity}

Testing the significance of the marginal effect difference component $\Delta_{M E}=\beta_{I V}-\beta_{C T}$ serves as a generalized Durbin-Wu-Hausman (DWH) test that is robust to the nonlinearity and observed heterogeneity of the treatment effects. This test further extends the generalized DWH test proposed in Lochner and Moretti (2015) by allowing for observed heterogeneity of the effects. Since $\widehat{\beta}_{I V}$ and $\widehat{\beta}_{C T}$ have a common denominator $\frac{1}{N} \sum_{i=1}^{N} \widetilde{X}_{i} \widetilde{Z}_{i}$, a relevant test statistic is

$$
\widehat{T}=\frac{\frac{1}{N} \sum_{i=1}^{N} d_{i} \widetilde{Z}_{i}}{\widehat{S}}
$$

\footnotetext{
${ }^{26}$ Another correction term appears in the case with DID- or RD-based instruments if $\widehat{b}_{O L S}\left(W_{i}\right)$ and $\widehat{\alpha}_{k}\left(W_{i}\right)$ are not specified to be linear, as presented in Appendix C.4.
} 
where $d_{i}=\widetilde{Y}_{i}-\widetilde{Y}_{2 i}$ is the difference in (residualized) dependent variables between two regressions that yield $\widehat{\beta}_{I V}$ and $\widehat{\beta}_{C T}$. $\widehat{S}$ is the standard error of the numerator, $\frac{1}{N} \sum_{i=1}^{N} d_{i} \widetilde{Z}_{i}$. For example, in an i.i.d. heteroscedastic case,

$$
N \widehat{S}^{2}=\frac{1}{N} \sum_{i=1}^{N}\left(d_{i} \widetilde{Z}_{i}-\frac{1}{N} \sum_{j=1}^{N} d_{j} \widetilde{Z}_{j}-v_{1 i} \widehat{\widetilde{Z}}_{i}\right)^{2}
$$

Under a more general distributional assumption, the right hand side of (29) should be replaced by the estimated asymptotic variance of $\frac{1}{\sqrt{N}} \sum_{i=1}^{N}\left(d_{i} \widetilde{Z}_{i}-\frac{1}{N} \sum_{j=1}^{N} d_{j} \widetilde{Z}_{j}-v_{1 i} \widehat{\widetilde{Z}}_{i}\right)$. Appendix C.5 shows that $\widehat{T}$ converges in distribution to $N(0,1)$ under the null hypothesis $\Delta_{M E}=0$ and diverges under the alternative hypothesis $\Delta_{M E} \neq 0.27$

Three limitations of this test are worth noting. First, it is a valid test of endogeneity only when the setup in Section 2 describes the true model. Most importantly, the marginal effect difference $\Delta_{M E}$ cannot be attributed to endogeneity bias alone in the presence of unobserved heterogeneity, as discussed in Section 3.2.28 Second, this test cannot detect endogeneity bias if the difference between the IV-identified and OLS-identified marginal effects $\frac{\partial}{\partial x} g(x, w)-\frac{\partial}{\partial x} m(x, w)$ in some regions of $(x, w)$ exactly cancels out the difference in other regions. While the direction of endogeneity bias is expected to be unambiguous in many economic contexts, one might consider the nonparametric test proposed by Blundell and Horowitz (2007) in the contexts in which the direction of endogeneity may differ across $(x, w)$. Finally, this test shares the fundamental limitations with the standard DWH test that the instrument must be valid and that it has little power to detect endogeneity bias if the instrument is not sufficiently strong 29

\footnotetext{
${ }^{27}$ As described in footnote 9, in a setting with multiple instruments $\left(Z_{i 1}, \ldots, Z_{i M}\right)$, the scalar instrument $Z_{i}$ is generated as $Z_{i}=\sum_{m=1}^{M} \pi_{m} Z_{i m}$. In this case, instead of performing the $t$-test with the synthetic $Z_{i}$, one could separately compute $\frac{1}{N} \sum_{i=1}^{N} d_{i} \widetilde{Z}_{i m}$ for each $m=1, \ldots, M$ and perform a chi-squared test to improve efficiency.

${ }^{28}$ In a binary treatment context, one might consider the frameworks such as Donald et al. (2014) and Mogstad et al. (2018), which can test endogeneity bias even with unobserved heterogeneity under several additional conditions.

${ }^{29}$ The asymptotic property of the test itself would not be influenced by the weak instrument problem, since the test statistic in (28) does not depend on the first stage coefficient, $\frac{1}{N} \sum_{i=1}^{N} \widetilde{X}_{i} \widetilde{Z}_{i}$. Staiger and Stock (1997) show that one version of the DWH test (Durbin, 1954) is robust to the weak instrument problem but two others (Hausman, 1978; Wu, 1973) are not.
} 


\section{Applications to Return-to-Schooling Estimates}

This section describes the empirical applications of my framework to return-to-schooling estimates with three different identification strategies 30 First, I use geographic variation in college costs as an instrument as in Cameron and Taber (2004) and Carneiro et al. (2011) and estimate the returns to schooling in the National Longitudinal Survey of Youth 1979 (NLSY79). Next, I exploit a discontinuity in the minimum school-leaving age in the United Kingdom as in Oreopoulos (2006), using the British General Household Survey (GHS). Finally, I exploit DID variation in compulsory schooling laws across cohorts and states in the United States as in Acemoglu and Angrist (2000) and estimate the returns to schooling in the 1960-1980 U.S. Censuses. Appendix D provides additional details for these analyses.

\subsection{College Cost Instrument with Geographic Variation}

This analysis uses the civilian sample from the NLSY79. The original sample consists of 5,579 males and 5,827 females born in 1957-64. After dropping persons with missing information about their Armed Forces Qualification Test (AFQT) score or county of residence at age 14, persons who have not completed 8th grade by age 22 , and persons with no wage information in any year, my sample consists of 4,719 males and 4,986 females. For each person, I use observations between ages 25 and 54 . The outcome $Y$ is the log hourly wage at the current or most recent job and the treatment $X$ is years of schooling top-coded at 18 years.

Because minority and economically disadvantaged households are sampled at higher rates, persons are weighted by the sampling weights throughout my analysis. Within each person, I use equal weights for the person-year observations 31 It is important to weight observations appropriately to recover the population OLS and IV coefficients because my framework does not assume the linear structural equation and admits a weighted-average interpretation of the coefficients.

As instruments, I use measures of direct and opportunity costs of college attendance as in Cameron and Taber (2004) and Carneiro et al. (2011) 32 In particular, I use the presence of a public four-year college and tuition rate of the nearest in-state public four-year college

\footnotetext{
${ }^{30}$ I use the term "returns to schooling" to refer to the causal effect of schooling on log wages, even though it sometimes denotes the internal rate of return associated with schooling in its most narrow sense.

${ }^{31} \mathrm{As}$ a result, the weight on a person-year observation is the sampling weight for the person divided by the number of years for which the person is observed.

${ }^{32}$ While I follow their identification strategies in estimating the causal effects of schooling, I do not follow their empirical specifications in my analysis. Their original analyses do not exactly fit into my framework because years of schooling is not the only endogenous variable in Cameron and Taber (2004) and the schooling measure is binary in Carneiro et al. (2011).
} 
in the county of residence at age 14 to represent the direct cost of attendance 33 While the tuition rate captures a pecuniary cost of attendance, college proximity captures both pecuniary (due to reduced costs of room and board) and nonpecuniary costs of attendance. I use local earnings and unemployment rate in the county of residence at age 14 in the year in which the person turns age 17 (1974 for the oldest cohort and 1981 for the youngest) to represent the opportunity cost of attendance 34

For the vector of covariates $W$, I use the AFQT percentile, age, an indicator for female, black and Hispanic dummies, indicators for parental education, the number of siblings, and cohort dummies 35 In addition, I include urban status, Census division dummies, and the average local earnings and unemployment rate during 1974-81 of the county of residence at age 14 in the covariate vector $W$. Using the average local labor market conditions as control variables ensures that variation in the corresponding instruments is driven by a temporary shock to the local labor market. Otherwise, the instruments may capture a permanent difference in local labor market conditions, which can be directly associated with potential earnings. Controlling for local labor market conditions is also important to ensure the plausibility of the college proximity instrument, as emphasized by Cameron and Taber (2004). Appendix D.1 provides further details of the sample and presents the first-stage regression results.

Table 1 reports the decomposition results. The first three columns of the table report the linear OLS coefficient, the linear IV coefficient, and the IV-OLS coefficient gap. The next three columns report the estimates of the covariate weight difference, the treatmentlevel weight difference, and the marginal effect difference. Here, I discuss the first row of the table, which reports the results from the NLSY79. The second and third rows are discussed in Sections 5.2 and 5.3. In the first row, the point estimates of the OLS and IV coefficients are nearly identical, with the OLS coefficient of 0.065 and the IV coefficient of 0.062. An empirical researcher may be tempted to conclude from this result that there is no evidence of ability bias in these data. However, the decomposition using the IVweighted OLS coefficients indicates that the IV coefficient would be well below the OLS coefficient if they had the same weights on the covariates and treatment levels. In fact, the covariate weight difference is estimated to be 0.011 and the treatment-level weight difference is estimated to be 0.018. With the weight difference components accounted for, the marginal

\footnotetext{
${ }^{33}$ In earlier work, Card $(\underline{1995 b})$ and Kane and Rouse (1995) use college proximity as the schooling instrument associated with the direct costs of college attendance. They do not use opportunity cost measures in their analyses.

${ }^{34}$ Local earnings are measured at the county level, while the unemployment rate is at the state level.

${ }^{35}$ The specification allows the AFQT scores to have different slopes across tertiles and the ages to have different slopes across the $25-34,35-44$, and $45-54$ age groups.
} 
effect difference is -0.032 , indicating that the IV-identified returns to schooling are lower than the OLS-identified returns. This result is rather consistent with the ability bias story in terms of point estimates, even though the generalized DWH test fails to reject at the $5 \%$ level given the large standard error.

I investigate the mechanisms underlying these results by examining the patterns of the IV and OLS weights. Table 2 presents the total IV and OLS weights on each group of covariates. The first three columns of the table present the population share, the total IV weight, and the total OLS weight on each group. Each set of weights sums to one across the whole sample by construction. The last column reports the OLS schooling coefficient from a linear regression performed separately for each group, to illustrate the difference in OLS-identified schooling effects across groups. While the OLS weights are close to the population shares, the IV weights are concentrated on persons with advantaged backgrounds in terms of AFQT score, parental education, and race/ethnicity. Schooling coefficients from the separately performed OLS regressions indicate that more-advantaged groups tend to have higher schooling effects. These data patterns result in the positive contribution of the covariate weight difference to the IV-OLS coefficient gap.

The pattern of IV weights is consistent with the empirical observation by Cameron and Taber (2004) that persons with advantaged backgrounds tend to be more sensitive to local college availability in the NLSY79. However, one may expect that persons with disadvantaged backgrounds should be weighted more because they are expected to be more sensitive to college cost instruments given their financial constraints 36 Several factors can explain low IV weights on persons with disadvantaged backgrounds. While Cameron and Taber (2004) suggest that increased funding on federal student aid programs in the 1970s is one such factor, low college attendance and graduation rates among persons from disadvantaged backgrounds can also be important 37 A low college attendance rate implies that the majority would be never-takers instead of compliers 38 A low college graduation rate implies that their completed years of schooling would not be strongly affected, even if the instruments affect their college attendance decisions.

Panel (a) of Figure 1 illustrates the total IV and OLS weights on each treatment margin. The IV weights are mostly placed on college education margins, with the weights on high

\footnotetext{
${ }^{36}$ Card $(1995 \mathrm{~b})$ and Kling (2001) find that persons from less advantaged backgrounds are more sensitive to the presence of a local college in the sample from the National Longitudinal Survey of Young Men, which is based on older cohorts than the NLSY79.

${ }^{37}$ The share of persons with one or more and four or more years of college education are $19 \%$ and $4 \%$, respectively, among the bottom third of AFQT scores. Among persons in the top third of AFQT scores, $80 \%$ have one or more years of college education and $56 \%$ have four or more years of college education.

${ }^{38}$ If the college enrollment decision is explained by a logit or probit model, the share of compliers is the largest among the group with a college attendance rate of $50 \%$.
} 
school margins close to zero. This weight pattern is consistent with the expectation that college cost instruments affect years of schooling through college attendance decisions. Higher IV weights on college education margins result in the positive contribution of the weight difference to the IV-OLS coefficient gap because marginal effects of years in college are much higher than marginal effects of years in high school in this sample. In fact, the linear OLS coefficient is 0.031 in the subsample with 12 or fewer years of schooling and 0.071 in the subsample with 12 or more years of schooling.

The overall result from this decomposition exercise that the IV coefficient is inflated due to the weight difference appears to match the discount rate bias argument developed by Lang (1993) and Card (1995a) 39 However, the estimated weight patterns indicate that the underlying mechanism is distinct in two ways. First, the IV coefficient places more weight on advantaged rather than disadvantaged groups in the population. Higher IV weights on advantaged groups give rise to the higher IV coefficient because of the higher marginal returns among advantaged groups. This is the opposite mechanism to the discount rate bias argument, even though it shifts the IV coefficient in the same direction. Second, higher marginal returns to college education give rise to the higher IV coefficient in this sample, given the concentration of IV weights on college years relative to high school years. The discount rate bias argument does not consider this possibility, as it assumes nonincreasing marginal returns to schooling.

\subsection{RD-Based Compulsory Schooling Instrument}

As in Oreopoulos (2006), I now exploit the 1947 compulsory schooling reform in the United Kingdom to construct an RD instrument. The U.K. government raised the minimum schoolleaving age in Great Britain from 14 to 15 years in 1947. The share of people leaving school at age 14 or earlier then fell from $56 \%$ in the 1932 birth cohort turning 14 one year before the reform to $9 \%$ in the 1934 birth cohort turning 14 one year after the reform.

The empirical strategy in this analysis closely follows Oreopoulos (2006). 40 I use the sample of persons younger than 65 years old from the British General Household Surveys in 1984-98 who turned 14 in 1935-65. I exclude persons with missing data on earnings or education and persons leaving school before age 10. The outcome $Y$ is log annual earnings.

\footnotetext{
${ }^{39}$ Building on the canonical model of Becker (1967), they consider a model in which individuals invest in education as long as the marginal return to an additional year of schooling exceeds its marginal cost. The model predicts that the marginal return at the chosen schooling level would be higher for credit-constrained individuals with higher discount rates. Given this prediction, they argue that the IV coefficient could exceed the population average return if credit-constrained individuals are more sensitive to the instruments and thus weighted more.

${ }^{40} \mathrm{My}$ regression results slightly differ from the originally published results in Oreopoulos (2006) due to the data correction (Oreopoulos, 2008) and a top-coding treatment of schooling described below.
} 
The treatment $X$ is years of schooling, which is given by the age when they left full-time education minus five years, with the top-coding at 20 years 41 The instrument $Z$ is an indicator for 1933 or later birth cohorts, who turned 14 in 1947 or later. The covariates $W$ are the fourth-order polynomials of birth cohort and age 42

The second row of Table 1 reports the decomposition of the IV-OLS coefficient gap in this empirical application. As the OLS estimate lies above the IV estimate by 0.021 , a researcher who presumes the linear causal model may immediately interpret it as the result of ability bias. However, adjusting for the weight difference suggests otherwise. In fact, the decomposition result indicates that the marginal returns identified by the IV coefficient exceed those identified by the OLS coefficient by 0.023 , after accounting for the covariate weight difference and the treatment-level weight difference components. The empirical result is no longer consistent with the ability bias story as a point estimate, although both standard and generalized DWH tests fail to reject at the $5 \%$ level due to the imprecise IV estimate.

Table 3 presents the IV and OLS weights on the birth cohorts. The IV weights are concentrated on the birth cohorts turning age 14 in 1941-50. The linear regression restricted to these cohorts yields smaller OLS schooling coefficients than those for the younger cohorts, who receive most of the OLS weights. This explains the negative contribution of the covariate weight difference to the IV-OLS coefficient gap. Panel (b) of Figure 1 shows that the IV weights are almost exclusively placed on the 10th year of schooling, which is exactly what the 1947 reform mandated. This weight pattern pushes down the IV coefficient because the 10th year of schooling has a smaller marginal return than the other schooling margins. In fact, the linear regression restricted to individuals with 9 or 10 years of schooling yields an OLS schooling coefficient of 0.037 , which is much smaller than the full-sample OLS coefficient.

This analysis demonstrates that researchers should exercise caution in extending the intuition of Imbens and Angrist (1994) to an empirical setting with covariates and a multivalued treatment. Observing a sharp decline in the dropout rate at age 14 after the reform, Oreopoulos (2006) argues that the IV and OLS coefficients in this setting are expected to identify the treatment effects for the comparable population, providing an analogy to the comparison between the LATE and ATE. In principle, however, the LATE interpretation in Imbens and Angrist (1994) draws on a model with a binary treatment and no covariates. It

\footnotetext{
${ }^{41}$ Approximately $2.5 \%$ of persons report having left full-time education after age 25 , and their schooling levels are all treated as 20 years. Oreopoulos (2006) does not make this top-coding treatment. See Appendix D.2 for the analysis without top-coding, which reaches the same conclusion regarding the relevance of the weight difference components.

${ }^{42}$ Gelman and Imbens (2019) recommend against the use of high-order polynomials in RD designs; nevertheless, I follow the original specification in Oreopoulos (2006). Using two separate quadratic polynomials for pre- and post-reform cohorts instead of global fourth-order polynomials slightly pushes up the IV estimate. However, it does not affect the finding that the weight difference components are important.
} 
is still plausible that the LATE and ATE in this setting match conditional on the schooling level and the birth cohort, judging by the extensive response to the reform. Nevertheless, the estimated patterns of the weights suggest that the IV and OLS coefficients identify the effects for entirely different birth cohorts at completely distinct schooling margins.

\subsection{Compulsory Schooling Instrument with DID Variation}

This analysis exploits DID variation in compulsory schooling laws across cohorts and regions, following Acemoglu and Angrist (2000) 43 The analysis sample consists of 40-49-year-old white males born in the United States from the 1960-80 Censuses. I use log weekly earnings as the outcome $Y$, and limit my analysis to persons with positive earnings and working for at least one week in the previous year. I use years of schooling as the treatment $X$. I include in a vector of covariates $W$ indicators for the state of birth and indicators for the year of birth. As instruments, I use the status of compulsory schooling laws (CSL) at age 14 in the state of birth. As in Acemoglu and Angrist (2000), I construct the CSL instruments based on the required years of schooling associated with child labor laws. Appendix D.3 provides further details of the sample, first-stage regression results, and decomposition results using other common compulsory schooling instruments.

The third row of Table 1 presents the estimated OLS and IV schooling coefficients and the decomposition of the IV-OLS coefficient gap. While the IV estimate is slightly above the OLS estimate with a coefficient gap of 0.017 , the decomposition result indicates that the gap is primarily associated with the weight difference components. Although the IV-OLS gap is not statistically distinguishable from zero even without accounting for the weight difference components, this result reshapes the quantitative implication from the gap. In particular, Acemoglu and Angrist (2000) attribute the small positive IV-OLS gap to modest external returns to schooling. Accounting for the weight difference components, this result indicates even less important externality than their original interpretation.

Table 4 presents the total IV and OLS weights on each group of covariates. The IV weights attached to some birth cohorts or birth states are negative. In fact, the IV weights aggregate to negative values among persons in the 1930-39 birth cohorts and among persons born in the Midwest or West. Moreover, I find that $54 \%$ of covariate-specific IV weights are negative and sum to -5.93 .

In a usual IV setting, negative weights imply the presence of both compliers and defiers.

\footnotetext{
${ }^{43} \mathrm{My}$ specification follows one of their main specifications in estimating private returns to schooling that uses the 1960-80 Census data with the child labor laws instrument and no state-of-residence controls (Acemoglu and Angrist, 2000, p.34). While they adjust some variables in the 1960-80 data to incorporate the 1950 Census data in their alternative specifications, I do not make these adjustments as I focus only on the 1960-80 Censuses. See Appendix D.3 for details.
} 
In a DID setting, however, the weights are not guaranteed to be positive even with the perfect compliance $X=Z$, as pointed out by de Chaisemartin and D'Haultfoeuille (2020) in the binary treatment case 44 While the IV-weighted OLS coefficient $\beta_{C}=\int b_{O L S}(w) \bar{\omega}_{Z}(w) d F_{W}(w)$ is a weighted average of the covariate-specific OLS coefficients $b_{O L S}(w)$, negative weights can push $\beta_{C}$ out of the support of $b_{O L S}(w)$. In fact, the estimates of $b_{O L S}(w)$ are no greater than 0.076 , despite $\beta_{C}$ being estimated to be 0.079 . This result suggests that even a small heterogeneity in treatment effects can give rise to a large contribution of the weight difference to the IV-OLS coefficient gap if the IV strategy relies on DID variation.

Panel (c) of Figure 1 reports the total IV and OLS weights for each schooling level. The IV weights mostly capture the schooling margins up to the 12 th year of schooling, which is consistent with the context that primary and secondary education is mandated by the CSL. However, the effect of this treatment-level weight difference on the IV-OLS coefficient gap is mostly obscured by the large contribution of the covariate weight difference.

\section{Conclusion}

When OLS and IV estimates differ, empirical researchers typically consider two explanations. The first takes the linear regression equation literally and interprets the coefficient gap as endogeneity bias. The second extends the intuition of the LATE interpretation (Imbens and Angrist, 1994) to a general regression equation and interprets the coefficient gap as the weight difference. My paper enables researchers to proceed a step further and formally quantify the contributions of the weight difference and endogeneity bias components separately.

I show that the IV-OLS coefficient gap is explained by differences in the weights on the covariates, the weights on the treatment levels, and the identified marginal effects. The marginal effect difference component captures endogeneity bias in the absence of the unobservable-driven interaction between the treatment effects and treatment responses to the instrument. I propose a simple two-step regression approach to perform the decomposition empirically, which can be implemented in standard statistical packages.

I demonstrate the practical value of my framework through its empirical applications to return-to-schooling estimates with compulsory schooling and college cost instruments. The IV-OLS coefficient gaps in these empirical applications are substantially influenced by the weight difference components, and accounting for them leads to different conclusions about the direction or extent of endogeneity bias.

\footnotetext{
${ }^{44}$ In fact, the first stage regression indicates the positive relationship between the CSL requirements and schooling levels, even in the subsample of the 1930-39 birth cohorts or among persons born in the Midwest or West.
} 


\section{References}

Acemoglu, Daron and Joshua Angrist (2000). "How large are human-capital externalities? Evidence from compulsory schooling laws". NBER Macroeconomics Annual 15, pp. 9-59.

Ackerberg, Daniel, Xiaohong Chen, and Jinyong Hahn (2012). "A practical asymptotic variance estimator for two-step semiparametric estimators". Review of Economics and Statistics 94.2 , pp. 481-498.

Angrist, Joshua D (1998). "Estimating the labor market impact of voluntary military service using social security data on military applicants". Econometrica, pp. 249-288.

Angrist, Joshua D, Kathryn Graddy, and Guido W Imbens (2000). "The interpretation of instrumental variables estimators in simultaneous equations models with an application to the demand for fish". The Review of Economic Studies 67.3, pp. 499-527.

Angrist, Joshua D and Guido W Imbens (1995). "Two-stage least squares estimation of average causal effects in models with variable treatment intensity". Journal of the American Statistical Association 90.430, pp. 431-442.

Angrist, Joshua D and Alan B Krueger (1991). "Does Compulsory School Attendance Affect Schooling and Earnings?" The Quarterly Journal of Economics 106.4, pp. 979-1014.

- (1999). "Empirical strategies in labor economics". Handbook of Labor Economics 3, pp. 12771366.

Angrist, Joshua D and Jörn-Steffen Pischke (2010). "The credibility revolution in empirical economics: How better research design is taking the con out of econometrics". Journal of Economic Perspectives 24.2, pp. 3-30.

Aronow, Peter M and Cyrus Samii (2016). "Does regression produce representative estimates of causal effects?" American Journal of Political Science 60.1, pp. 250-267.

Becker, Gary Stanley (1967). Human Capital and the Personal Distribution of Income; an Analytical Approach, by Gary S. Becker. Institute of Public Administration.

Black, Sandra E, Paul J Devereux, and Kjell G Salvanes (2005). "Why the apple doesn't fall far: Understanding intergenerational transmission of human capital". American Economic Review 95.1, pp. 437-449.

Blundell, Richard, Xiaohong Chen, and Dennis Kristensen (2007). "Semi-nonparametric IV estimation of shape-invariant Engel curves". Econometrica 75.6, pp. 1613-1669.

Blundell, Richard and Joel L Horowitz (2007). "A non-parametric test of exogeneity". The Review of Economic Studies 74.4, pp. 1035-1058. 
Cameron, Stephen V and Christopher Taber (2004). "Estimation of educational borrowing constraints using returns to schooling". Journal of Political Economy 112.1, pp. 132-182.

Card, David (1995a). "Earnings, Schooling, and Ability Revisited". In: ed. by Solomon W. Polachek. Vol. 14. Greenwich Connecticut: JAI Press.

- (1995b). "Using Geographic Variation in College Proximity to Estimate the Return to Schooling". In: Toronto: University of Toronto Press.

- (1999). "The causal effect of education on earnings". In: Handbook of Labor Economics. Vol. 3. Elsevier, pp. 1801-1863.

- (2001). "Estimating the return to schooling: Progress on some persistent econometric problems". Econometrica 69.5, pp. 1127-1160.

Carneiro, Pedro, James J Heckman, and Edward Vytlacil (2010). "Evaluating marginal policy changes and the average effect of treatment for individuals at the margin". Econometrica 78.1, pp. 377-394.

Carneiro, Pedro, James J Heckman, and Edward J Vytlacil (2011). "Estimating marginal returns to education". American Economic Review 101.6, pp. 2754-81.

Chernozhukov, Victor, Guido W Imbens, and Whitney K Newey (2007). "Instrumental variable estimation of nonseparable models". Journal of Econometrics 139.1, pp. 4-14.

de Chaisemartin, Clément and Xavier D'Haultfoeuille (2018). "Fuzzy differences-in-differences". The Review of Economic Studies 85.2, pp. 999-1028.

- (2020). "Two-Way Fixed Effects Estimators with Heterogeneous Treatment Effects." American Economic Review 110.9, pp. 2964-2996.

Donald, Stephen G, Yu-Chin Hsu, and Robert P Lieli (2014). "Testing the unconfoundedness assumption via inverse probability weighted estimators of (L)ATT". Journal of Business E) Economic Statistics 32.3, pp. 395-415.

Duflo, Esther (2001). "Schooling and labor market consequences of school construction in Indonesia: Evidence from an unusual policy experiment". American Economic Review 91.4, pp. 795-813.

Durbin, James (1954). "Errors in Variables". Review of the International Statistical Institute 22 , pp. 23-32.

Gelman, Andrew and Guido Imbens (2019). "Why high-order polynomials should not be used in regression discontinuity designs". Journal of Business $\&$ Economic Statistics 37.3, pp. 447-456.

Hausman, Jerry A (1978). "Specification tests in econometrics". Econometrica 46.6, pp. 12511271. 
Heckman, James J and Sergio Urzua (2010). "Comparing IV with structural models: What simple IV can and cannot identify". Journal of Econometrics 156.1, pp. 27-37.

Heckman, James J, Sergio Urzua, and Edward Vytlacil (2006). "Understanding instrumental variables in models with essential heterogeneity". The Review of Economics and Statistics 88.3, pp. 389-432.

Heckman, James J and Edward Vytlacil (2001). "Policy-relevant treatment effects". American Economic Review 91.2, pp. 107-111.

Horowitz, Joel L (2011). "Applied nonparametric instrumental variables estimation". Econometrica 79.2, pp. 347-394.

Imbens, Guido W and Joshua D Angrist (1994). "Identification and estimation of local average treatment effects". Econometrica 62.2, pp. 467-475.

Imbens, Guido W and Whitney K Newey (2009). "Identification and estimation of triangular simultaneous equations models without additivity". Econometrica 77.5, pp. 1481-1512.

Kane, Thomas J and Cecilia Elena Rouse (1995). "Labor-market returns to two-and four-year college". The American Economic Review 85.3, pp. 600-614.

Kling, Jeffrey R (2001). "Interpreting instrumental variables estimates of the returns to schooling". Journal of Business \& Economic Statistics 19.3, pp. 358-364.

Lang, Kevin (1993). "Ability bias, discount rate bias and the return to education".

Lochner, Lance and Enrico Moretti (2001). "The Effect of Education on Crime: Evidence from Prison Inmates, Arrests, and Self-Reports". NBER Working Paper No. 8605.

- (2015). "Estimating and testing models with many treatment levels and limited instruments". Review of Economics and Statistics 97.2, pp. 387-397.

Løken, Katrine V, Magne Mogstad, and Matthew Wiswall (2012). "What linear estimators miss: The effects of family income on child outcomes". American Economic Journal: Applied Economics 4.2, pp. 1-35.

Masten, Matthew A and Alexander Torgovitsky (2016). "Identification of instrumental variable correlated random coefficients models". Review of Economics and Statistics 98.5, pp. 1001-1005.

Mogstad, Magne, Andres Santos, and Alexander Torgovitsky (2018). "Using instrumental variables for inference about policy relevant treatment parameters". Econometrica 86.5, pp. $1589-1619$.

Mogstad, Magne, Alexander Torgovitsky, and Christopher R. Walters (2021). "The Causal Interpretation of Two-Stage Least Squares with Multiple Instrumental Variables". American Economic Review 111.11, pp. 3663-98. 
Mogstad, Magne and Matthew Wiswall (2010). "Linearity in instrumental variables estimation: Problems and solutions". IZA Discussion Paper No. 5216.

Newey, Whitney K and Daniel McFadden (1994). "Large sample estimation and hypothesis testing". Handbook of Econometrics 4, pp. 2111-2245.

Newey, Whitney K and James L Powell (2003). "Instrumental variable estimation of nonparametric models". Econometrica 71.5, pp. 1565-1578.

Oreopoulos, Philip (2006). "Estimating average and local average treatment effects of education when compulsory schooling laws really matter". American Economic Review 96.1, pp. $152-175$.

- (2008). "Estimating average and local average treatment effects of education when compulsory schooling laws really matter: Corrigendum". American Economic Review.

Schechtman, E and S Yitzhaki (2004). "The Gini Instrumental Variable, or the "double instrumental variable" estimator". Metron, pp. 287-313.

Słoczyński, Tymon (2020). "Interpreting OLS Estimands When Treatment Effects Are Heterogeneous: Smaller Groups Get Larger Weights". The Review of Economics and Statistics.

- (2021). "When Should We (Not) Interpret Linear IV Estimands as LATE?"

Staiger, Douglas and James H Stock (1997). "Instrumental Variables Regression with Weak Instruments". Econometrica 65.3, pp. 557-586.

Wu, De-Min (1973). "Alternative Tests of Independence between Stochastic Regressors and Disturbances". Econometrica 41.4, pp. 733-750.

Yitzhaki, Shlomo (1996). "On using linear regressions in welfare economics". Journal of Business 86 Economic Statistics 14.4, pp. 478-486. 
Table 1: Decomposition of the IV-OLS Gap in Return-to-Schooling Estimates

\begin{tabular}{|c|c|c|c|c|c|c|c|}
\hline \multirow{2}{*}{ IV Strategy } & \multirow{2}{*}{ Data } & \multicolumn{3}{|c|}{ Coefficients } & \multicolumn{3}{|c|}{ Decomposition } \\
\hline & & OLS & IV & IV-OLS & $\overline{\Delta_{C W}}$ & $\overline{\Delta_{T W}}$ & $\Delta_{M E}$ \\
\hline $\begin{array}{l}\text { College Cost } \\
\text { Variation }\end{array}$ & NLSY79 ${ }^{1)}$ & $\begin{array}{c}0.065 \\
(0.003)\end{array}$ & $\begin{array}{c}0.062 \\
(0.087)\end{array}$ & $\begin{array}{l}-0.004 \\
(0.087)\end{array}$ & $\begin{array}{c}0.011 \\
(0.011)\end{array}$ & $\begin{array}{c}0.018 \\
(0.010)\end{array}$ & $\begin{array}{l}-0.032 \\
(0.086)\end{array}$ \\
\hline $\begin{array}{c}\text { Compulsory } \\
\text { Schooling RD }\end{array}$ & British GHS ${ }^{2)}$ & $\begin{array}{c}0.084 \\
(0.002)\end{array}$ & $\begin{array}{c}0.062 \\
(0.083)\end{array}$ & $\begin{array}{l}-0.021 \\
(0.082)\end{array}$ & $\begin{array}{l}-0.016 \\
(0.009)\end{array}$ & $\begin{array}{l}-0.029 \\
(0.018)\end{array}$ & $\begin{array}{c}0.023 \\
(0.078)\end{array}$ \\
\hline $\begin{array}{l}\text { Compulsory } \\
\text { Schooling DID }\end{array}$ & U.S. Census ${ }^{3)}$ & $\begin{array}{c}0.067 \\
(0.0004)\end{array}$ & $\begin{array}{c}0.084 \\
(0.022)\end{array}$ & $\begin{array}{c}0.017 \\
(0.022)\end{array}$ & $\begin{array}{c}0.011 \\
(0.004)\end{array}$ & $\begin{array}{c}0.003 \\
(0.003)\end{array}$ & $\begin{array}{c}0.003 \\
(0.021)\end{array}$ \\
\hline
\end{tabular}

Notes: Standard errors are in parentheses. The first three columns report the OLS estimates, the IV estimates, and their gaps. The next three columns report the estimates of the covariate weight difference, the treatment-level weight difference, and the marginal effect difference components. By construction, these three components sum to the IV-OLS gap. Appendix D.4 describes the empirical specification for estimating the decomposition.

1) Standard errors are robust to heteroskedasticity and correlation across observations on persons living in the same county at age 14. The instruments are college cost measures as defined in the main text.

2) Standard errors are robust to heteroskedasticity and correlation across observations on the same birth cohort and survey year. The instrument is an indicator for turning age 14 in 1947 or later.

3) Standard errors are robust to heteroskedasticity and correlation across observations on the same state and year of birth. The instruments are indicators for compulsory schooling requirements implied by child labor laws $(7,8$, and 9 or more years). 
Table 2: The IV and OLS Weights on the Covariate Groups (NLSY79)

\begin{tabular}{cccccc}
\hline Variable & Group & $\begin{array}{c}\text { Group } \\
\text { share }\end{array}$ & $\begin{array}{c}\text { OLS } \\
\text { weight }\end{array}$ & $\begin{array}{c}\text { IV } \\
\text { weight }\end{array}$ & $\begin{array}{c}\text { Subsample } \\
\text { OLS coef. }\end{array}$ \\
\hline \multirow{2}{*}{ AFQT } & $0-1 / 3$ & 0.32 & $0.25(0.01)$ & $-0.08(0.16)$ & $0.056(0.006)$ \\
percentile & $1 / 3-2 / 3$ & 0.34 & $0.35(0.01)$ & $0.19(0.13)$ & $0.062(0.005)$ \\
& $2 / 3-1$ & 0.34 & $0.40(0.01)$ & $0.89(0.20)$ & $0.074(0.005)$ \\
\hline Highest & Some HS of less & 0.24 & $0.21(0.01)$ & $-0.12(0.15)$ & $0.055(0.005)$ \\
parental & HS graduate & 0.42 & $0.41(0.01)$ & $0.66(0.17)$ & $0.069(0.005)$ \\
education & Some college & 0.34 & $0.37(0.01)$ & $0.46(0.15)$ & $0.068(0.005)$ \\
\hline \multirow{2}{*}{ Race/Ethnicity } & Black & 0.14 & $0.14(0.02)$ & $-0.12(0.11)$ & $0.069(0.005)$ \\
& Hispanic & 0.06 & $0.06(0.01)$ & $0.02(0.03)$ & $0.064(0.006)$ \\
Sex & Other & 0.80 & $0.80(0.02)$ & $1.10(0.11)$ & $0.064(0.004)$ \\
\hline Urban residence & Male & 0.50 & $0.49(0.01)$ & $0.47(0.17)$ & $0.057(0.004)$ \\
at age 14 & Rural & 0.31 & $0.30(0.03)$ & $0.53(0.17)$ & $0.073(0.006)$ \\
Region & Urban & 0.69 & $0.70(0.03)$ & $0.47(0.17)$ & $0.062(0.004)$ \\
\hline at age 14 & Northeast & 0.22 & $0.22(0.04)$ & $0.18(0.14)$ & $0.061(0.007)$ \\
& Midwest & 0.31 & $0.30(0.04)$ & $0.51(0.17)$ & $0.070(0.007)$ \\
& South & 0.32 & $0.32(0.04)$ & $0.09(0.13)$ & $0.058(0.005)$ \\
& West & 0.15 & $0.16(0.03)$ & $0.23(0.15)$ & $0.071(0.005)$ \\
\hline
\end{tabular}

Notes: Standard errors are in parentheses and robust to heteroskedasticity and correlation across observations on persons living in the same county at age 14. Each set of weights sums to one across the whole sample. Subsample OLS coefficient of years of schooling is obtained with the same set of control variables as regressions in Table 1, Appendix D.4 describes the empirical specification for estimating the weights. 
Table 3: The IV and OLS Weights on the Covariate Groups (British GHS)

\begin{tabular}{ccccc}
\hline Year at 14 & $\begin{array}{c}\text { Group } \\
\text { share }\end{array}$ & $\begin{array}{c}\text { OLS } \\
\text { weights }\end{array}$ & $\begin{array}{c}\text { IV } \\
\text { weights }\end{array}$ & $\begin{array}{c}\text { Subsample } \\
\text { OLS coef. }\end{array}$ \\
\hline $1935-40$ & 0.04 & 0.03 & -0.09 & 0.054 \\
& & $(0.01)$ & $(0.05)$ & $(0.012)$ \\
$1941-45$ & 0.09 & 0.08 & 0.68 & 0.065 \\
& & $(0.01)$ & $(0.15)$ & $(0.005)$ \\
$1946-50$ & 0.14 & 0.12 & 0.49 & 0.079 \\
& & $(0.02)$ & $(0.06)$ & $(0.006)$ \\
$1951-55$ & 0.19 & 0.18 & -0.10 & 0.086 \\
& & $(0.02)$ & $(0.17)$ & $(0.004)$ \\
$1956-60$ & 0.25 & 0.26 & 0.05 & 0.088 \\
& & $(0.03)$ & $(0.07)$ & $(0.003)$ \\
$1961-65$ & 0.28 & 0.34 & -0.02 & 0.088 \\
& & $(0.03)$ & $(0.05)$ & $(0.003)$ \\
\hline
\end{tabular}

Notes: Standard errors (in parentheses) are robust to heteroskedasticity and correlation across observations on the same birth cohort and survey year. Each set of weights sums to one across the whole sample. Subsample OLS coefficient of years of schooling is obtained with controlling for quartic terms of ages and birth cohorts. Appendix D.4 describes the empirical specification for estimating the weights. 
Table 4: The IV and OLS Weights on the Covariate Groups (U.S. Census)

\begin{tabular}{|c|c|c|c|c|c|}
\hline Variable & Group & $\begin{array}{l}\text { Group } \\
\text { share }\end{array}$ & $\begin{array}{c}\text { OLS } \\
\text { weights }\end{array}$ & $\begin{array}{c}\text { IV } \\
\text { weights }\end{array}$ & $\begin{array}{c}\text { Subsample } \\
\text { OLS coef. }\end{array}$ \\
\hline \multirow{3}{*}{$\begin{array}{l}\text { Year of } \\
\text { birth }\end{array}$} & 1910-19 & 0.32 & $\begin{array}{c}0.33 \\
(0.02)\end{array}$ & $\begin{array}{l}1.56 \\
(0.25)\end{array}$ & $\begin{array}{c}0.063 \\
(0.001)\end{array}$ \\
\hline & 1920-29 & 0.35 & $\begin{array}{c}0.36 \\
(0.02)\end{array}$ & $\begin{array}{l}1.25 \\
(0.28)\end{array}$ & $\begin{array}{c}0.070 \\
(0.001)\end{array}$ \\
\hline & 1930-39 & 0.33 & $\begin{array}{c}0.31 \\
(0.02)\end{array}$ & $\begin{array}{l}-1.81 \\
(0.38)\end{array}$ & $\begin{array}{c}0.067 \\
(0.001)\end{array}$ \\
\hline \multirow{4}{*}{$\begin{array}{l}\text { Region of } \\
\text { birth }\end{array}$} & Northeast & 0.29 & $\begin{array}{c}0.26 \\
(0.02)\end{array}$ & $\begin{array}{c}0.24 \\
(0.37)\end{array}$ & $\begin{array}{c}0.069 \\
(0.001)\end{array}$ \\
\hline & Midwest & 0.33 & $\begin{array}{c}0.27 \\
(0.02)\end{array}$ & $\begin{array}{l}-1.32 \\
(0.34)\end{array}$ & $\begin{array}{c}0.066 \\
(0.001)\end{array}$ \\
\hline & South & 0.30 & $\begin{array}{c}0.39 \\
(0.02)\end{array}$ & $\begin{array}{l}2.95 \\
(0.41)\end{array}$ & $\begin{array}{c}0.068 \\
(0.001)\end{array}$ \\
\hline & West & 0.09 & $\begin{array}{c}0.08 \\
(0.01)\end{array}$ & $\begin{array}{l}-0.87 \\
(0.15)\end{array}$ & $\begin{array}{c}0.064 \\
(0.001)\end{array}$ \\
\hline
\end{tabular}

Notes: Standard errors (in parentheses) are robust to heteroskedasticity and correlation across observations on the same state and year of birth. Each set of weights sums to one across the whole sample. Subsample OLS coefficient of years of schooling is obtained with controlling for state of birth and year of birth dummies. Appendix D.4 describes the empirical specification for estimating the weights. 
Figure 1: The IV and OLS Weights on the Treatment Levels

(a) NLSY79 (College Costs IV)

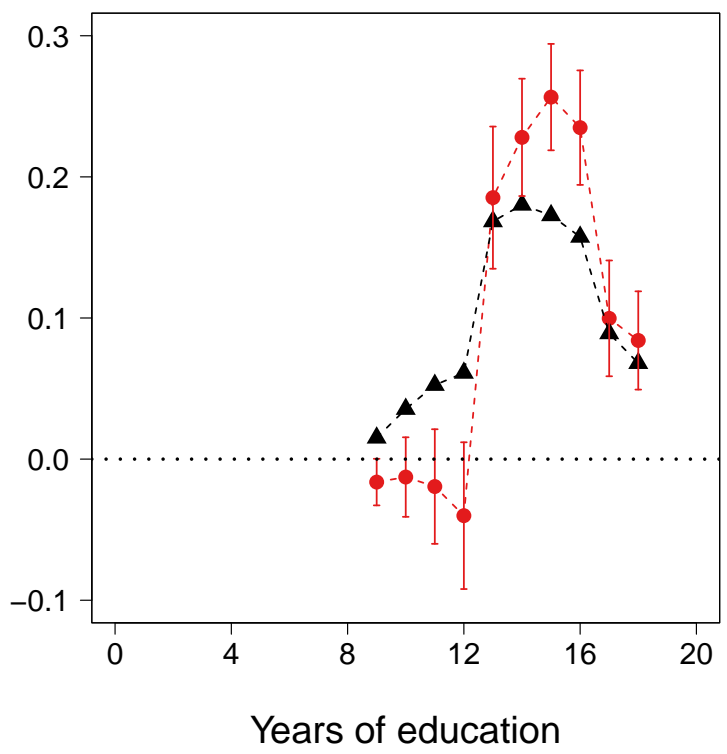

(c) U.S. Census (Compulsory Schooling DID)

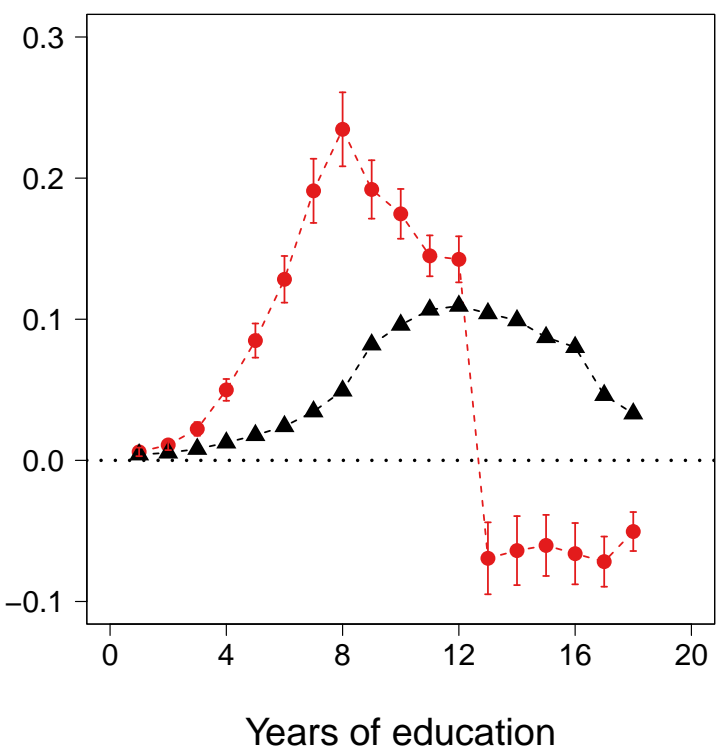

(b) British GHS (Compulsory Schooling RD)

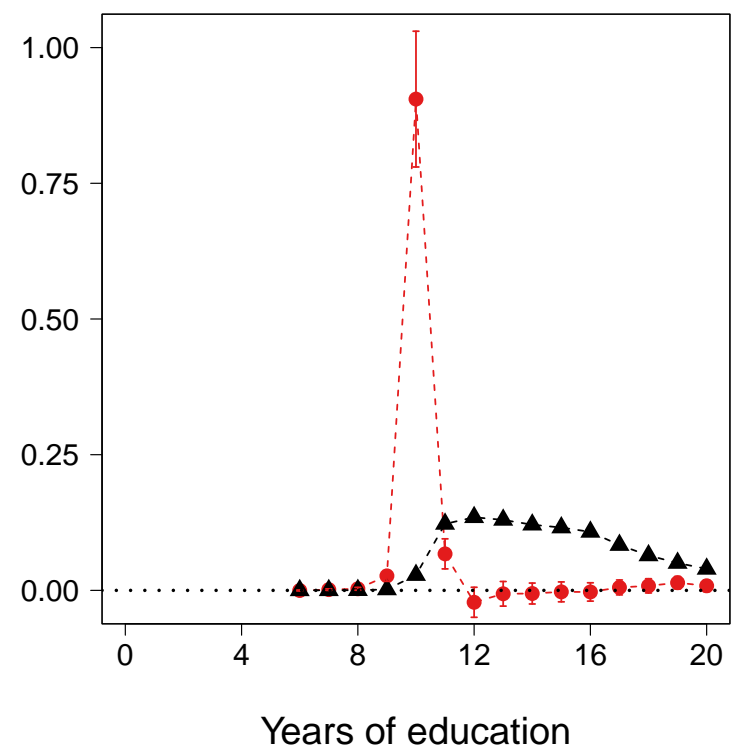

- - IV weight $\quad-$-OLS weight

Notes: The IV weights are presented with standard error bars. The standard error bars for the OLS weights are omitted because they are graphically negligible. Each set of weights sums to one across the whole sample. Appendix D.4 describes the empirical specification for estimating the weights. The weights are zero outside the support of years of education by construction, and thus are not plotted on the graph. 


\title{
Online Appendix: Empirical Decomposition of the IV-OLS Gap with Heterogeneous and Nonlinear Effects
}

\author{
Shoya Ishimaru*
}

\section{A Proofs}

This section provides proofs of key theorems. In the main paper, any integral expression of $x$ is taken over the support $(\underline{x}, \bar{x})$, but it is kept implicit to simplify the exposition. In this Appendix, it is kept explicit for rigorousness.

Some proofs rely on the following lemmas.

Lemma 1. Suppose $g:(\underline{x}, \bar{x}) \mapsto \mathbb{R}$ is a differentiable function, where $-\infty \leq \underline{x}<\bar{x} \leq \infty$. Then $\int_{\underline{x}}^{\bar{x}} g^{\prime}(x)\left(\mathbb{1}_{b \geq x}-\mathbb{1}_{a>x}\right) d x=g(b)-g(a)$ for any $a, b \in(\underline{x}, \bar{x})$.

Proof. It immediately follows from the fundamental theorem of calculus, given the identity that $\mathbb{1}_{b \geq x}-\mathbb{1}_{a>x}$ is equal to 1 if $a \leq x \leq b$, to -1 if $b<x<a$, and to 0 otherwise.

Lemma 2. Suppose a random variable $X$ satisfies Assumption $Q$. Let $R$ be any random variable and $W$ be any random vector, and define $\widetilde{R}=R-W^{\prime} E\left[W W^{\prime}\right]^{-1} E[W R]$. Let $h(x, w)$ be a function differentiable in $x$, and suppose that the total variation $V_{a}^{b}(h, w)=$ $\int_{\min \{a, b\}}^{\max \{a, b\}}\left|\frac{\partial}{\partial x} h(x, w)\right| d x$ satisfies $E\left[V_{x_{0}}^{X}(h, W)^{2}\right]<\infty$ for some $x_{0} \in(\underline{x}, \bar{x})$. If $E(R \mid W=w)$ is linear in $w$ or $h(x, w)$ is linear in $w$ for each $x \in(\underline{x}, \bar{x})$, then

$$
E(h(X, W) \widetilde{R})=\int_{\underline{x}}^{\bar{x}} \int \frac{\partial}{\partial x} h(x, w) E\left(\mathbb{1}_{X \geq x} \widetilde{R} \mid W=w\right) d F_{W}(w) d x .
$$

Proof. Take any $x_{0} \in(\underline{x}, \bar{x})$. Then,

$$
\begin{aligned}
E(h(X, W) \widetilde{R}) & =E\left[\left(h(X, W)-h\left(x_{0}, W\right)\right) \widetilde{R}\right] \\
& =E\left[\left(\int_{\underline{x}}^{\bar{x}} \frac{\partial}{\partial x} h(x, W)\left(\mathbb{1}_{X \geq x}-\mathbb{1}_{x_{0}>x}\right) d x\right) \widetilde{R}\right]
\end{aligned}
$$

The first equality follows from the linearity of $h\left(x_{0}, w\right)$ or $E[R \mid W=w]$ in $w$. Applying Lemma 1 by letting $b=X$ and $a=x_{0}$ (for each point in the sample space) yields the second equality. Note that $(\underline{x}, \bar{x})$ can be unbounded when applying Lemma 1 , as each realization of $X$ is finite even if its support $(\underline{x}, \bar{x})$ is unbounded.

\footnotetext{
*Hitotsubashi University, Department of Economics (email: shoya.ishimaru@r.hit-u.ac.jp).
} 
Continuing the transformation,

$$
\begin{aligned}
E(h(X, W) \widetilde{R}) & =\int_{\underline{x}}^{\bar{x}} E\left[\frac{\partial}{\partial x} h(x, W)\left(\mathbb{1}_{X \geq x}-\mathbb{1}_{x_{0}>x}\right) \widetilde{R}\right] d x \\
& =\int_{\underline{x}}^{\bar{x}} E\left[\frac{\partial}{\partial x} h(x, W) \mathbb{1}_{X \geq x} \widetilde{R}\right] d x \\
& =\int_{\underline{x}}^{\bar{x}} \int \frac{\partial}{\partial x} h(x, w) E\left(\mathbb{1}_{X \geq x} \widetilde{R} \mid W=w\right) d F_{W}(w) d x
\end{aligned}
$$

The first equality uses Fubini's theorem. The second equality follows from the linearity of $h(x, w)$ or $E[R \mid W=w]$ in $w$. The third equality follows from the law of iterated expectations. Note that Fubini's theorem can be applied here because

$$
\begin{aligned}
E\left[\int_{\underline{x}}^{\bar{x}}\left|\frac{\partial}{\partial x} h(x, W)\left(\mathbb{1}_{X \geq x}-\mathbb{1}_{x_{0}>x}\right) \widetilde{R}\right| d x\right] & =E\left[|\widetilde{R}| \cdot \int_{\underline{x}}^{\bar{x}}\left|\frac{\partial}{\partial x} h(x, W)\right|\left|\mathbb{1}_{X \geq x}-\mathbb{1}_{x_{0}>x}\right| d x\right] \\
& =E\left[|\widetilde{R}| \cdot V_{x_{0}}^{X}(h, W)\right] \\
& \leq \sqrt{E\left[\widetilde{R}^{2}\right] E\left[V_{x_{0}}^{X}(h, w)^{2}\right]} \\
& <\infty
\end{aligned}
$$

where the second equality follows from the identity that $\left|\mathbb{1}_{X \geq x}-\mathbb{1}_{x_{0}>x}\right|$ is equal to 1 if $x$ is between $x_{0}$ and $X$ and to 0 otherwise. This completes the proof.

\section{A.1 Proof of Theorem 1}

Proof. The IV coefficient is given by

$$
\begin{aligned}
\beta_{I V} & =\frac{E(\widetilde{Y} \widetilde{Z})}{E(\widetilde{X} \widetilde{Z})} \\
& =\frac{E[g(X, W) \widetilde{Z}]}{E(\widetilde{X} \widetilde{Z})} \\
& =\int_{\underline{x}}^{\bar{x}} \int \frac{\partial}{\partial x} g(x, w) \frac{E\left(\mathbb{1}_{X \geq x} \widetilde{Z} \mid W=w\right)}{E(\widetilde{X} \widetilde{Z})} d F_{W}(w) d x \\
& =\int_{\underline{x}}^{\bar{x}} \int \frac{\partial}{\partial x} g(x, w) \omega_{Z}(x, w) d F_{W}(w) d x .
\end{aligned}
$$

The second equality uses Assumptions $[\mathrm{S}$ and IV-(i). The third equality uses Lemma 2 by letting $R=Z$ and $h=g$, where Assumptions $\mathrm{D}-(\mathrm{i})$ and $\mathrm{L}-(\mathrm{i})$ are required to use the lemma. 
The last equality uses Assumption $\mathrm{L}$-(i).

Since $E[Y \widetilde{X}]=E[E[Y \mid X, W] \widetilde{X}]=E[m(X, W) \widetilde{X}]$, letting $R=X$ and $h=m$ in Lemma 2 gives the weighted-average expression for $\beta_{O L S}$ based on the same argument as above.

Finally, letting $Y=X$ gives $\frac{\partial}{\partial x} g(x, w)=1$ and $\beta_{I V}=1$ in (A.1), which jointly yield $1=\int_{\underline{x}}^{\bar{x}} \int \omega_{Z}(x, w) d F_{W}(w) d x$. Letting $Y=X$ also gives $\frac{\partial}{\partial x} m(x, w)=1$ and $\beta_{O L S}=1$, which jointly imply $1=\int_{\underline{x}}^{\bar{x}} \int \omega_{X}(x, w) d F_{W}(w) d x$.

\section{A.2 Proof of Theorem 2}

Proof. $E[Y \widetilde{Z}]=E\left[b_{I V}(W) X \widetilde{Z}\right]$ follows from the equalities below.

$$
\begin{aligned}
E\left[\left(Y-b_{I V}(W) X\right) \widetilde{Z}\right] & =E\left[\left(Y-\frac{\operatorname{Cov}(Y, Z \mid W)}{\operatorname{Cov}(X, Z \mid W)} X\right) \widetilde{Z}\right] \\
& =E\left[E(Y \widetilde{Z} \mid W)-\frac{\operatorname{Cov}(Y, Z \mid W)}{\operatorname{Cov}(X, Z \mid W)} E(X \widetilde{Z} \mid W)\right] \\
& =E[\operatorname{Cov}(Y, Z \mid W)-\operatorname{Cov}(Y, Z \mid W)] \\
& =0 .
\end{aligned}
$$

The first equality uses the definition of $b_{I V}(w)$, the second equality uses the law of iterated expectations, and the third equality uses Assumption $\mathbb{L}$-i). Given $E[Y \widetilde{Z}]=E\left[b_{I V}(W) X \widetilde{Z}\right]$, letting $h(x, w)=b_{I V}(w) x$ in Lemma 2 yields $\beta_{I V}=\int b_{I V}(w) \bar{\omega}_{Z}(w) d F_{W}(w)$. The same argument yields $\beta_{O L S}=\int b_{O L S}(w) \bar{\omega}_{X}(w) d F_{W}(w)$ by letting $Z=X$.

\section{A.3 Proof of Theorem 3}

Proof. Using Theorem 1 conditional on $W=w$ immediately gives these results. See also Yitzhaki (1996) and Schechtman and Yitzhaki (2004).

\section{A.4 Proof of Theorem 4}

Proof. Since $E[Y \widetilde{Z}]=E[g(X, W) \widetilde{Z}]$ due to Assumptions $\leqq$ and IV-(i), letting $R=Z$ and $h=g$ in Lemma 2 gives the following weighted-average expression for $\beta_{I V}$, where Assumptions D-(i) and LS are required to use the lemma.

$$
\beta_{I V}=\int_{\underline{x}}^{\bar{x}} \int \frac{\partial}{\partial x} g(x, w) \frac{E\left(\mathbb{1}_{X \geq x} \widetilde{Z} \mid W=w\right)}{E(\widetilde{X} \widetilde{Z})} d F_{W}(w) d x .
$$


Since $\frac{\partial}{\partial x} g(x, w)$ is linear in $w$ for each $x \in(\underline{x}, \bar{x})$ due to Assumption $\llbracket \underline{\operatorname{LS}}, \frac{E\left(\mathbb{1}_{X>x} \widetilde{Z} \mid W=w\right)}{E(\widetilde{X} \widetilde{Z})} \operatorname{can}$ be replaced with its linear projection $\omega_{Z}^{*}(x, w)=L_{w}\left(\mathbb{1}_{X \geq x} \widetilde{Z}\right) / E(\widetilde{X} \widetilde{Z})$, as well as any function $r(x, w)$ with $L_{w}(r(x, W))=\omega_{Z}^{*}(x, w)$. Finally, letting $Y=X$ in (A.2) gives $\frac{\partial}{\partial x} g(x, w)=1$ and $\beta_{I V}=1$, which jointly yield $\int_{\underline{x}}^{\bar{x}} \int \omega_{Z}^{*}(x, w) d F_{W}(w) d x=1$.

\section{A.5 Proof of Theorem 5}

Proof. Take any $x_{0} \in(\underline{x}, \bar{x})$. Then,

$$
\begin{aligned}
E(Y \widetilde{Z}) & =E\left[\left(Y(X)-Y\left(x_{0}\right)\right) \widetilde{Z}\right] \\
& =E\left[\left(\int_{\underline{x}}^{\bar{x}} Y^{\prime}(x)\left(\mathbb{1}_{X \geq x}-\mathbb{1}_{x_{0}>x}\right) d x\right) \widetilde{Z}\right] \\
& =\int_{\underline{x}}^{\bar{x}} E\left[Y^{\prime}(x)\left(\mathbb{1}_{X \geq x}-\mathbb{1}_{x_{0}>x}\right) \tilde{Z}\right] d x \\
& =\int_{\underline{x}}^{\bar{x}} E\left[Y^{\prime}(x) \mathbb{1}_{X \geq x} \widetilde{Z}\right] d x .
\end{aligned}
$$

The first and last equalities follow from Assumptions $\mathrm{P}-(\mathrm{i})$ and $\mathrm{L}-(\mathrm{i})$. Applying Lemma 1 by letting $b=X$ and $a=x_{0}$ (for each point in the sample space) yields the second equality. The third equality uses Fubini's theorem, which can be applied because

$$
\begin{aligned}
E\left[\int_{\underline{x}}^{\bar{x}}\left|Y^{\prime}(x)\left(\mathbb{1}_{X \geq x}-\mathbb{1}_{x_{0}>x}\right) \widetilde{Z}\right| d x\right] & =E\left[|\widetilde{Z}| \cdot \int_{\min \left\{x_{0}, X\right\}}^{\max \left\{x_{0}, X\right\}}\left|Y^{\prime}(x)\right| d x\right] \\
& =E\left[V_{x_{0}}^{X}(Y(\cdot))|\widetilde{Z}|\right] \\
& \leq \sqrt{E\left[V_{x_{0}}^{X}(Y(\cdot))^{2}\right] E\left[\widetilde{Z}^{2}\right]} \\
& <\infty .
\end{aligned}
$$

Continuing the transformation,

$$
\begin{aligned}
E(Y \widetilde{Z}) & =\int_{\underline{x}}^{\bar{x}} E\left[E\left[Y^{\prime}(x) E\left[\mathbb{1}_{X \geq x} \widetilde{Z} \mid Y^{\prime}(x), W\right] \mid W\right]\right] d x \\
& =\int_{\underline{x}}^{\bar{x}} E\left[E\left[Y^{\prime}(x) \lambda\left(Y^{\prime}(x) \mid x, W\right) \mid W\right] \omega_{Z}(x, W)\right] d x \\
& =\int_{\underline{x}}^{\bar{x}} \int \tau_{I V}(x, w) \omega_{Z}(x, w) d F_{W}(w) d x .
\end{aligned}
$$

The first equality uses the law of iterated expectations. The second equality follows from 
the definition of the weight functions $\lambda$ and $\omega_{Z}$. The last equality uses the definition of $\tau_{I V}(x, w)$. This completes the proof.

\section{B Additional Extensions}

This section provides additional econometric results that extend the analyses in the main paper.

\section{B.1 When Linearity Is Not a Good Approximation}

Except for DID and RD designs, Assumption $\mathrm{L}$ can be made as close an approximation as possible with sufficient data. However, it can be restrictive in empirical applications with a limited sample size and many covariates, in which it is difficult to make a covariate vector sufficiently flexible. Without the assumption, both $\beta_{I V}$ and $\beta_{O L S}$ may have omitted variable bias associated with unaccounted nonlinear effects of covariates.

Removing Assumption $\mathrm{L}$ from Theorem 1 yields the following result.

Theorem 6. Let $\omega_{R}(x, w)=\frac{\operatorname{Cov}\left(\mathbb{1}_{X \geq x}, R \mid W=w\right)}{E\left[\operatorname{Cov}\left(\mathbb{1}_{X \geq x}, R \mid W\right)\right]}$ for $R=Z, X$.

(i) With Assumptions $\left[, \quad, \quad, D-(i)\right.$, and $I V$, the difference between the IV coefficient $\beta_{I V}$ and the weighted average

$$
\beta_{I V}^{*}=\int_{\underline{x}}^{\bar{x}} \int \frac{\partial}{\partial x} g(x, w) \omega_{Z}(x, w) d F_{W}(w) d x
$$

is given by

$$
\beta_{I V}-\beta_{I V}^{*}=\frac{E\left[\left(E\left(Y-\beta_{I V}^{*} X \mid W\right)-L_{W}\left(Y-\beta_{I V}^{*} X\right)\right)\left(E(Z \mid W)-L_{W}(Z)\right)\right]}{E[\widetilde{X} \widetilde{Z}]} .
$$

(ii) With Assumptions $\square, D$-(ii), and $O L S$, the difference between the OLS coefficient $\beta_{O L S}$ and the weighted average

$$
\beta_{O L S}^{*}=\int_{\underline{x}}^{\bar{x}} \int \frac{\partial}{\partial x} m(x, w) \omega_{X}(x, w) d F_{W}(w) d x
$$

is given by

$$
\beta_{O L S}-\beta_{O L S}^{*}=\frac{E\left[\left(E\left(Y-\beta_{O L S}^{*} X \mid W\right)-L_{W}\left(Y-\beta_{O L S}^{*} X\right)\right)\left(E(X \mid W)-L_{W}(X)\right)\right]}{E\left[\widetilde{X}^{2}\right]} .
$$


Theorem [implies that the weighted-average interpretation of the IV and OLS coefficients remains valid but it accompanies approximation errors associated with omitted nonlinear effects of covariates $W 45$ Nonetheless, it is still possible to estimate the weight difference components, $\Delta_{C W}$ and $\Delta_{T W}$, using parametric approximations of weights $\omega_{Z}(x, w)$ and $\omega_{X}(x, w)$ and effects $b_{O L S}(w)$ and $\frac{\partial}{\partial x} m(x, w)$. Thus, the decomposition continues to provide relevant information about how much the weight difference matters for the IV-OLS coefficient gap. However, it may also require caution when interpreting the remaining gap because the approximation errors, as well as endogeneity bias, can influence the gap.

The proof of Theorem [6 is presented below.

Proof. By construction, $\beta_{I V}^{*}$ is given by

$$
\begin{aligned}
\beta_{I V}^{*} & =\int_{\underline{x}}^{\bar{x}} \int \frac{\partial}{\partial x} g(x, w) \omega_{Z}(x, w) d F_{W}(w) d x \\
& =\int b_{I V}(w) \bar{\omega}_{Z}(w) d F_{W}(w) \\
& =\frac{E[\operatorname{Cov}(Y, Z \mid W)]}{E[\operatorname{Cov}(X, Z \mid W)]} \\
& =\frac{E[(Y-E(Y \mid W))(Z-E(Z \mid W))]}{E[(X-E(X \mid W))(Z-E(Z \mid W))]} .
\end{aligned}
$$

Then,

$$
\begin{aligned}
E[\tilde{Y} \widetilde{Z}]= & E\left[(Y-E(Y \mid W))(Z-E(Z \mid W))+\left(E(Y \mid W)-L_{W}(Y)\right)\left(E(Z \mid W)-L_{W}(Z)\right)\right] \\
= & \beta_{I V}^{*} E[(X-E(X \mid W))(Z-E(Z \mid W))]+E\left[\left(E(Y \mid W)-L_{W}(Y)\right)\left(E(Z \mid W)-L_{W}(Z)\right)\right] \\
= & \beta_{I V}^{*} E\left[\widetilde{X} \widetilde{Z}-\left(E(X \mid W)-L_{W}(X)\right)\left(E(Z \mid W)-L_{W}(Z)\right)\right] \\
& +E\left[\left(E(Y \mid W)-L_{W}(Y)\right)\left(E(Z \mid W)-L_{W}(Z)\right)\right] \\
= & \beta_{I V}^{*} E[\widetilde{X} \widetilde{Z}]+E\left[\left(E\left(Y-\beta_{I V}^{*} X \mid W\right)-L_{W}\left(Y-\beta_{I V}^{*} X\right)\right)\left(E(Z \mid W)-L_{W}(Z)\right)\right]
\end{aligned}
$$

Note that the second equality uses (A.3) and the other ones follow from the law of iterated expectations and simple algebra. Therefore, $\beta_{I V}$ can be expressed as follows.

$$
\begin{aligned}
\beta_{I V} & =E(\tilde{Y} \widetilde{Z}) / E(\widetilde{X} \widetilde{Z}) \\
& =\beta_{I V}^{*}+\frac{E\left[\left(E\left(Y-\beta_{I V}^{*} X \mid W\right)-L_{W}\left(Y-\beta_{I V}^{*} X\right)\right)\left(E(Z \mid W)-L_{W}(Z)\right)\right]}{E(\widetilde{X} \widetilde{Z})}
\end{aligned}
$$

\footnotetext{
${ }^{45}$ It is conceptually possible to isolate the approximation error by estimating a model that allows for a greater flexibility in the covariate vector $W$. In this situation, however, one could use the framework in Section 2 by estimating a more flexible model in the first place.
} 
The same argument yields the expression for $\beta_{O L S}$ by letting $Z=X$.

\section{B.2 Decomposition with Invalid Instrument}

Allowing the instrument to be correlated with the unobservable $U$ by removing Assumption IV-(i), the interpretation of the IV coefficient changes as follows.

Theorem 7. With Assumptions $[S, \square, \square, \Pi D-(i i)$, and $\square-(i)$, the linear IV coefficient is

$$
\beta_{I V}=\int_{\underline{x}}^{\bar{x}} \int \frac{\partial}{\partial x} g(x, w) \omega_{Z}(x, w) d F_{W}(w) d x+\frac{E[U \widetilde{Z}]}{E[X \widetilde{Z}]} .
$$

Proof. The proof is virtually identical to that of Theorem 1.

Because of the correlation between the instrument and the unobservable $U$, the IV coefficient is biased from the weighted average of the causal effects. The interpretation of the decomposition (14 16) does not change except for (16), which is replaced by

$$
\Delta_{M E}=\int_{\underline{x}}^{\bar{x}} \int\left(\frac{\partial}{\partial x} g(x, w)-\frac{\partial}{\partial x} m(x, w)\right) \omega_{Z}(x, w) d F_{W}(w) d x+\frac{E[U \widetilde{Z}]}{E[X \widetilde{Z}]} .
$$

The first term still captures the dependence of the treatment $X$ on the unobservable $U$, as implied by (6) . The newly added second term captures the correlation between the instrument $Z$ and the unobservable $U$ after controlling for covariates $W$

\section{B.3 Decomposition with Unobserved Heterogeneity and DID/RD- Based Identification}

While Section 3 allows for either a DID/RD-based identification or unobserved heterogeneity, this extension allows for both at the same time. Starting from the setting in Section [3.2, I remove Assumption $\mathrm{L}$, which does not hold for DID and RD cases. Then, I make the following assumption on the potential outcome $Y(x)$.

Assumption LP. (Linearity of Conditional Potential Outcomes) $E[Y(x) \mid W=w]$ is linear in $w$ for each $x \in(\underline{x}, \bar{x})$.

\footnotetext{
${ }^{46}$ This correlation can arise from both the failure of exclusion restriction and the failure of random assignment, although this analysis does not distinguish between them. For example, $U$ could be decomposed as $U=h(Z)+V$, where the failure of exclusion restriction implies $h \neq 0$ and the failure of random assignment implies $E(V \widetilde{Z}) \neq 0$.
} 
In a DID setting discussed in Section 3.1, Assumption LP can be written as $E[Y(x) \mid W]=$ $\sum_{g=1}^{G} \gamma_{g}(x) d_{g}+\sum_{t=1}^{T-1} \delta_{t}(x) D_{t}$, which implies parallel trends of the average potential outcome for each treatment level $x$. In an RD setting, Assumption LP can be written as $E[Y(x) \mid C]=\sum_{k=1}^{K} \gamma_{k}(x) p_{k}(C)$. This implies that the mean potential outcome conditional on a running variable $C$ is well approximated by a linear combination of the basis functions $\left(p_{1}(C), \ldots, p_{K}(C)\right)$, which also implies continuity at the cutoff point $C=c$. Note that Assumption $\mathrm{P}-(\mathrm{i})$ trivially holds in these settings since $\operatorname{Cov}(Y(x), Z \mid W)=0$ immediately follows from $\operatorname{Var}(Z \mid W)=0$.

Theorem 8. With Assumptions $\mathbb{P}, \square,[V-(i i)$, and $L P$, the linear IV regression coefficient is

$$
\beta_{I V}=\int_{\underline{x}}^{\bar{x}} \int \tau_{I V}(x, w) \omega_{Z}(x, w) d F_{W}(w) d x,
$$

where the IV-identified marginal effect $\tau_{I V}(x, w)$ at each $(x, w)$ is given by

$$
\tau_{I V}(x, w)=E\left[Y^{\prime}(x) \lambda\left(Y^{\prime}(x) \mid x, w\right) \mid W=w\right]
$$

with $\lambda(t \mid x, w)=\frac{E\left[\mathbb{1}_{X \geq x} \widetilde{Z} \mid Y^{\prime}(x)=t, W=w\right]}{E\left[\mathbb{1}_{X \geq x} \widetilde{Z} \mid W=w\right]}$ and $E\left[\lambda\left(Y^{\prime}(x) \mid x, w\right) \mid W=w\right]=1$. The weight function $\omega_{Z}(x, w)$ is given by

$$
\omega_{Z}(x, w)=\frac{E\left[\mathbb{1}_{X \geq x} \widetilde{Z} \mid W=w\right]}{E[X \widetilde{Z}]} .
$$

Two important implications of Theorem 8 are worth noting. First, the marginal effect difference component $\Delta_{M E}$ of the decomposition in (14-16) should be attributed to not only endogeneity bias but also the unobservable-driven weight difference, as discussed in Section 3.2 .

Second, unlike in Section [3.1, the weight function $\omega_{Z}(x, w)=\frac{E\left[\mathbb{1}_{X \geq x} \widetilde{Z} \mid W=w\right]}{E[X \widetilde{Z}]}$ cannot be reduced to its linearly projected counterpart $\omega_{Z}^{*}(x, w)=\frac{L_{w}\left(\mathbb{1}_{X \geq x} \widetilde{Z}\right)}{E(X \widetilde{Z})}$ in general. Even though the AME $\tau(x, w)=E\left[Y^{\prime}(x) \mid W=w\right]$ is linear in $w$ due to Assumption LP, the IV-identified marginal effect $\tau_{I V}(x, w)=E\left[Y^{\prime}(x) \lambda\left(Y^{\prime}(x) \mid x, w\right) \mid W=w\right]$ does not necessarily satisfy the linearity condition due to the weight function $\lambda\left(Y^{\prime}(x) \mid x, w\right)$ that depends on $w$.

An interesting special case of Theorem 8 is a sharp DID or RD with $X=Z$. In this case, the equation (A.4) simplifies to

$$
\beta_{I V}=\int_{\underline{x}}^{\bar{x}} \int \tau(x, w) \omega_{Z}^{*}(x, w) d F_{W}(w) d x .
$$


Even with unobserved heterogeneity, perfect compliance due to $X=Z$ eliminates the unobservable-driven weight difference. This makes the weighted-average expression essentially identical to Theorem 4 , which rules out unobserved heterogeneity.

The proof of Theorem 8 is presented below. This proof is similar to that of Theorem 5 , Proof. Take any $x_{0} \in(\underline{x}, \bar{x})$. Then,

$$
\begin{aligned}
E(Y \widetilde{Z}) & =E\left[\left(Y(X)-Y\left(x_{0}\right)\right) \widetilde{Z}\right] \\
& =E\left[\left(\int_{\underline{x}}^{\bar{x}} Y^{\prime}(x)\left(\mathbb{1}_{X \geq x}-\mathbb{1}_{x_{0}>x}\right) d x\right) \widetilde{Z}\right] \\
& =\int_{\underline{x}}^{\bar{x}} E\left[Y^{\prime}(x)\left(\mathbb{1}_{X \geq x}-\mathbb{1}_{x_{0}>x}\right) \widetilde{Z}\right] d x \\
& =\int_{\underline{x}}^{\bar{x}} E\left[Y^{\prime}(x) \mathbb{1}_{X \geq x} \widetilde{Z}\right] d x .
\end{aligned}
$$

The first and last equalities follow from Assumptions $\mathrm{P}-$ (i) and $\mathrm{LP}$. Applying Lemma 1 by letting $b=X$ and $a=x_{0}$ (for each point in the sample space) yields the second equality. The third equality uses Fubini's theorem.

Continuing the transformation,

$$
\begin{aligned}
E(Y \widetilde{Z}) & =\int_{\underline{x}}^{\bar{x}} E\left[E\left[Y^{\prime}(x) E\left[\mathbb{1}_{X \geq x} \widetilde{Z} \mid Y^{\prime}(x), W\right] \mid W\right]\right] d x \\
& =\int_{\underline{x}}^{\bar{x}} E\left[E\left[Y^{\prime}(x) \lambda\left(Y^{\prime}(x) \mid x, W\right) \mid W\right] \omega_{Z}(x, W)\right] d x \\
& =\int_{\underline{x}}^{\bar{x}} \int \tau_{I V}(x, w) \omega_{Z}(x, w) d F_{W}(w) d x .
\end{aligned}
$$

The first equality uses the law of iterated expectations. The second equality follows from the definition of the weight functions $\lambda$ and $\omega_{Z}$. The last equality uses the definition of $\tau_{I V}(x, w)$.

\section{Other Econometric Details}

This section provides technical details and additional econometric results omitted from the main paper. 


\section{C.1 Derivation of the Marginal OLS Weights on Treatment Levels}

The derivation of (11) uses the following lemma.

Lemma 3. Let $X$ be any random variable with mean $\mu_{X}$. Then, for any real number $x$,

$$
E\left[\mathbb{1}_{X \geq x}\left(X-\mu_{X}\right)\right]=\int_{\underline{x}}^{\bar{x}} \int_{\underline{x}}^{\bar{x}}\left(x_{1}-x_{2}\right) \mathbb{1}_{x_{2}<x \leq x_{1}} d F_{X}\left(x_{1}\right) d F_{X}\left(x_{2}\right) .
$$

Proof. As in Yitzhaki (1996), it is a well-known property of the U-statistics that $\operatorname{Cov}(Y, X)=$ $\frac{1}{2} E\left[\left(Y_{1}-Y_{2}\right)\left(X_{1}-X_{2}\right)\right]$, where $\left(X_{i}, Y_{i}\right) \stackrel{i . i . d .}{\sim} F_{X, Y}$ for $i=1,2$. Letting $Y=\mathbb{1}_{X \geq x}$ in this result yields

$$
\begin{aligned}
E\left[\mathbb{1}_{X \geq x}\left(X-\mu_{X}\right)\right] & =\frac{1}{2} E\left[\left(\mathbb{1}_{X_{1} \geq x}-\mathbb{1}_{X_{2} \geq x}\right)\left(X_{1}-X_{2}\right)\right] \\
& =E\left[\mathbb{1}_{X_{1}>X_{2}}\left(\mathbb{1}_{X_{1} \geq x}-\mathbb{1}_{X_{2} \geq x}\right)\left(X_{1}-X_{2}\right)\right] \\
& =\int_{\underline{x}}^{\bar{x}} \int_{\underline{x}}^{\bar{x}}\left(x_{1}-x_{2}\right) \mathbb{1}_{x_{2}<x \leq x_{1}} d F_{X}\left(x_{1}\right) d F_{X}\left(x_{2}\right) .
\end{aligned}
$$

This completes the proof.

Using Lemma [3, $\bar{\omega}_{X}(x)$ is given by

$$
\begin{aligned}
\bar{\omega}_{X}(x) & =\frac{E\left[\mathbb{1}_{X \geq x} \widetilde{X}\right]}{E\left[\widetilde{X}^{2}\right]} \\
& =\frac{E\left[E\left[\mathbb{1}_{X \geq x}(X-E[X \mid W]) \mid W\right]\right]}{E\left[\widetilde{X}^{2}\right]} \\
& =E\left[\widetilde{X}^{2}\right]^{-1} \iint_{\underline{x}}^{\bar{x}} \int_{\underline{x}}^{\bar{x}}\left(x_{1}-x_{2}\right) \mathbb{1}_{x_{2}<x \leq x_{1}} d F_{X}\left(x_{1} \mid w\right) d F_{X}\left(x_{2} \mid w\right) d F_{W}(w) .
\end{aligned}
$$

Note that the second equality uses Assumption $\mathrm{L}$-(i) and the law of iterated expectations. The last equality uses Lemma 3 conditional on $W=w$. This confirms the equation (11).

\section{C.2 Comparing the Weight Function with de Chaisemartin and D'Haultfoeuille (2020)}

Important special cases of Theorem 4 are DID settings studied by de Chaisemartin and D'Haultfoeuille (2020), in which $X$ is a binary treatment $(x=0,1)$ and a covariate vector consists of time and group dummies as $W=\left(d_{1}, \ldots, d_{G}, D_{1}, \ldots, D_{T-1}\right)$. They consider a 
sharp DID setting with $X=Z$ and a fuzzy DID setting in which $X \neq Z$. Using the notation in the main paper, the weight on the covariate value $W=w$ they suggest can be expressed as $\frac{E(X \tilde{Z} \mid W=w)}{E(X \tilde{Z})}$. This weight function differs from $\bar{\omega}_{Z}^{*}(w)=\frac{L_{w}(X \tilde{Z})}{E(X \tilde{Z})}$ suggested in Section 3.1 . The discrepancy of the weight functions arises because de Chaisemartin and D'Haultfoeuille (2020) assume a parallel trend only for a potential outcome without treatment in deriving the weights. The parallel trend assumption is usually justified by the absence of groupperiod specific economic shocks coinciding with the instrument $Z$. Thus, assuming parallel trends for both the treated and untreated outcomes is no more economically restrictive than assuming so only for the untreated outcome in many cases. In fact, de Chaisemartin and D'Haultfoeuille (2020) themselves assume a parallel trend for a potential outcome with treatment when they propose their alternative estimator. Note that the treatment effects are still allowed to be heterogeneous across groups and periods in an additive way.

Parallel trends for both treated and untreated outcomes imply the linearity of the treatment effect $g(1, w)-g(0, w)$. Therefore, their weight function $\frac{E(X \tilde{Z} \mid W=w)}{E(X \tilde{Z})}$ can be linearly projected onto $W$ without affecting the weighted average. The linear projection in this case means expressing the weight function as additive in group dummies $d_{g}$ and time dummies $D_{t}$. The projected weight function exactly matches the weight function $\bar{\omega}_{Z}^{*}(w)$ in Section 3.1 .

There are two important exceptions in which it can be reasonable to use the original weight function in de Chaisemartin and D'Haultfoeuille (2020) without linearly projecting it. The first exception arises when treatment effects are dynamic. In this situation, the treatment effect can depend on the number of periods since the treatment assignment in a nonlinear way. As a result, the treatment effect (and thus the weights) can be non-additive in group and period effects, as in Borusyak et al. (2021). The second exception can arise in a fuzzy DID setting due to a subtle effect of unobserved heterogeneity as discussed in Section B.3. Due to the unobservable-driven weight difference, the IV-identified marginal effect $\tau_{I V}(x, w)$ can be nonlinear in $w$ even if the $\operatorname{AME} \tau(x, w)$ is linear in $w$.

\section{C.3 Relation to the LATE and MTE Interpretations}

Section 3.2 explores the weighted-average interpretation of the IV coefficient without assuming that the treatment is monotonic in the instrument. This section considers the weightedaverage interpretation under the monotonicity condition, as in Angrist and Imbens (1995) and Heckman et al. (2006). Let $X(z)$ be the potential treatment associated with the instrument level $z$. The observed treatment is $X=X(Z)$. I make the following set of assumptions, modifying Assumption $\mathrm{P}$. 
Assumption $\mathbf{P}^{\prime}$. The process of the potential outcome and treatment $(Y(\cdot), X(\cdot))$ satisfies:

(i) (Conditional Independence) $(Y(\cdot), X(\cdot))$ and $Z$ are independent given $W$;

(ii) (Differentiability) $Y^{\prime}(x)$ exists and the total variation $V_{x_{0}}^{X}(Y(\cdot))=\int_{\min \left\{x_{0}, X\right\}}^{\max \left\{x_{0}, X\right\}}\left|Y^{\prime}(x)\right| d x$ satisfies $E\left[V_{x_{0}}^{X}(Y(\cdot))^{2}\right]$ for some $x_{0} \in(\underline{x}, \bar{x})$;

(iii) (Monotonicity) $X\left(z^{\prime}\right) \geq X(z)$ for any $z^{\prime} \geq z$.

Given the monotonicity condition, there exists a stochastic process $V(\cdot)$ (valued on the extended real line) that satisfies $X \geq x \Leftrightarrow Z \geq V(x)$ for any $x \in \mathbb{R}$. $V(x)$ is an inverse of $X(z)$ defined such that the treatment $X$ exceeds the level $x$ whenever the instrument $Z$ exceeds the cutoff $V(x)$. While such $V(x)$ is not unique if $X(z)$ is not strictly increasing or if $Z$ is discrete, how $V(x)$ is defined does not influence the following results.

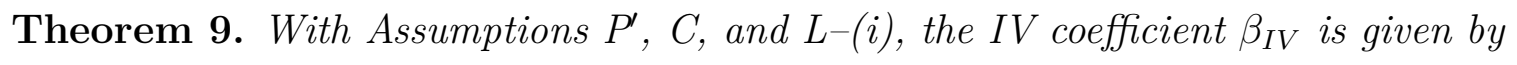

$$
\beta_{I V}=\int_{\underline{x}}^{\bar{x}} \int \tau_{I V}(x, w) \omega_{Z}(x, w) d F_{W}(w) d x,
$$

where the IV-identified marginal effect $\tau_{I V}(x, w)$ at each $(x, w)$ is expressed as

$$
\tau_{I V}(x, w)=\int E\left[Y^{\prime}(x) \mid V(x)=v, W=w\right] \kappa(v \mid x, w) d F_{V(x) \mid W}(v \mid w) .
$$

The weight function $\kappa(v \mid x, w)$ is

$$
\kappa(v \mid x, w)=\frac{\iint\left(z_{1}-z_{2}\right) \mathbb{1}_{z_{1} \geq v>z_{2}} d F_{Z \mid W}\left(z_{1} \mid w\right) d F_{Z \mid W}\left(z_{2} \mid w\right)}{\operatorname{Cov}\left(\mathbb{1}_{X \geq x}, Z \mid W\right)}
$$

and integrates to one: $\int \kappa(v \mid x, w) d F_{V(x) \mid W}(v \mid w)=1$.

Theorem 9 is merely a reinterpretation of Theorem 5 under the stronger monotonicity condition. The equation (A.5) represents the IV-identified effect $\tau_{I V}(x, w)$ using the unobserved threshold $V(x)$. This representation generalizes the weighted-average expression based on the marginal treatment effect (MTE) in Heckman et al. (2006) to the continuous treatment case.

The equation (A.5) can also be expressed using the pairwise LATE interpretation in Imbens and Angrist (1994), Angrist and Imbens (1995), and Angrist et al. (2000). 47 Rewriting

\footnotetext{
${ }^{47}$ Although the term "LATE" is sometimes exclusively reserved for a binary treatment case (in fact, Angrist and Imbens (1995) and Angrist et al. (2000) do not use this term), I use it here for the lack of a better expression.
} 
the equation yields

$$
\tau_{I V}(x, w)=\iint E\left[Y^{\prime}(x) \mid X\left(z_{1}\right) \geq x>X\left(z_{2}\right), W=w\right] \phi\left(z_{1}, z_{2} \mid x, w\right) d F_{Z \mid W}\left(z_{1} \mid w\right) d F_{Z}\left(z_{2} \mid w\right)
$$

where the weight on each $\left(z_{1}, z_{2}\right)$ is given by

$$
\phi\left(z_{1}, z_{2} \mid x, w\right)=\frac{\left(z_{1}-z_{2}\right) \operatorname{Pr}\left[X\left(z_{1}\right) \geq x>X\left(z_{2}\right) \mid W=w\right]}{\operatorname{Cov}\left(\mathbb{1}_{X \geq x}, Z \mid W=w\right)} .
$$

This expression implies that the IV-identified effect $\tau_{I V}(x, w)$ is the weighted average of pairwise LATE associated with two alternative instrument assignments, $Z_{1}, Z_{2} \stackrel{i . i . d .}{\sim} F_{Z \mid W}$. The weight on each pairwise LATE is proportional to the difference between two assignments and how likely the difference induces individuals to cross the treatment level $x .48$ If the instrument is binary, $\tau_{I V}(x, w)$ simplifies to the $(x, w)$-specific LATE: $\tau_{I V}(x, w)=$ $E\left[Y^{\prime}(x) \mid X(1) \geq x>X(0), W=w\right]$.

I now present the proof of Theorem 9,

Proof. This proof is given by slightly modifying the proof of Theorem 5 ,

$$
\begin{aligned}
E(Y \widetilde{Z}) & =\int_{\underline{x}}^{\bar{x}} E\left[Y^{\prime}(x) \mathbb{1}_{X \geq x} \widetilde{Z}\right] d x \\
& =\int_{\underline{x}}^{\bar{x}} E\left[Y^{\prime}(x) \mathbb{1}_{Z \geq V(x)} \widetilde{Z}\right] d x \\
& =\int_{\underline{x}}^{\bar{x}} \iint E\left[Y^{\prime}(x) \mathbb{1}_{Z \geq v} \widetilde{Z} \mid V(x)=v, W=w\right] d F_{V(x) \mid W}(v \mid w) d F_{W}(w) d x \\
& =\int_{\underline{x}}^{\bar{x}} \iint E\left[Y^{\prime}(x) \mid V(x)=v, W=w\right] E\left[\mathbb{1}_{Z \geq v} \widetilde{Z} \mid W=w\right] d F_{V(x) \mid W}(v \mid w) d F_{W}(w) d x
\end{aligned}
$$

The first equality is shown in the proof of Theorem 5. The second equality follows from the existence of $V(x)$ due to Assumption $\mathrm{P}^{\prime}$-(iii). The third equality uses the law of iterated expectations. The fourth equality follows from $V(x) \perp Z \mid W$ due to the definition of $V(x)$ and Assumption $\mathrm{P}^{\prime}-(\mathrm{i})$.

\footnotetext{
${ }^{48}$ The pairwise LATE interpretation in Imbens and Angrist (1994), Angrist and Imbens (1995), and Angrist et al. (2000) use adjacent pairs to construct the weighted average, which makes their weightedaverage expressions more difficult to interpret.
} 
Using Lemma 3 and Assumption —-(i), the weight function is given by

$$
\begin{aligned}
\kappa(v \mid x, w) & =\frac{E\left[\mathbb{1}_{Z \geq v} \widetilde{Z} \mid W=w\right]}{E\left[\mathbb{1}_{X \geq x} \tilde{Z} \mid W=w\right]} \\
& =\frac{\iint\left(z_{1}-z_{2}\right) \mathbb{1}_{z_{1} \geq v>z_{2}} d F_{Z \mid W}\left(z_{1} \mid w\right) d F_{Z \mid W}\left(z_{2} \mid w\right)}{\operatorname{Cov}\left(\mathbb{1}_{X \geq x}, Z \mid W\right)} .
\end{aligned}
$$

Finally, integrating the weight function $\kappa(v \mid x, w)$ over $v$ yields

$$
\begin{aligned}
\int \kappa(v \mid x, w) d F_{V(x) \mid W}(v \mid w) & =\frac{\int E\left[\mathbb{1}_{Z \geq v} \widetilde{Z} \mid W=w\right] d F_{V(x) \mid W}(v \mid w)}{E\left[\mathbb{1}_{X \geq x} \tilde{Z} \mid W=w\right]} \\
& =\frac{\int E\left[\mathbb{1}_{Z \geq v} \tilde{Z} \mid W=w, V(x)=v\right] d F_{V(x) \mid W}(v \mid w)}{E\left[\mathbb{1}_{X \geq x} \tilde{Z} \mid W=w\right]} \\
& =\frac{E\left[\mathbb{1}_{Z \geq V(x)} \tilde{Z} \mid W=w\right]}{E\left[\mathbb{1}_{X \geq x} \tilde{Z} \mid W=w\right]} \\
& =1,
\end{aligned}
$$

where the second equality follows from $V(x) \perp Z \mid W$.

\section{C.4 Statistical Properties of the Decomposition Estimators}

The following discussion focuses on the statistical properties of $\widehat{\beta}_{C T}$, since the estimation of $\widehat{\beta}_{C}$ can be considered as its special case. To simplify the exposition, I use $\widehat{\beta}$ to denote $\widehat{\beta}_{C T}$. I focus on the parametric case, given the equivalence result in Ackerberg et al. (2012).

Let $\left(Y_{i}, X_{i}, Z_{i}, W_{i}\right)_{i=1}^{N}$ be an i.i.d. random sample that satisfies the set of assumptions in Section 2, The first step solves the minimization problem

$$
\min _{\theta} \frac{1}{N} \sum_{i=1}^{N}\left(Y_{i}-B_{i}^{\prime} \theta\right)^{2},
$$

where $B_{i}^{\prime}=\left(q_{k 1}\left(W_{i}\right) P_{i k}, \ldots, q_{k L^{(k)}}\left(W_{i}\right) P_{i k}\right)_{k=1}^{K}$ is a vector that stacks the basis functions and $\theta^{\prime}=\left(\theta_{k 1}, \ldots, \theta_{k L^{(k)}}\right)_{k=1}^{K}$ is a vector that stacks the parameters. This yields the vector moment condition

$$
0=\frac{1}{N} \sum_{i=1}^{N} B_{i}\left(Y_{i}-B_{i}^{\prime} \hat{\theta}\right) .
$$


Then, the second step solves for the scalar moment condition $0=\frac{1}{N} \sum_{i=1}^{N} h_{i}(\widehat{\beta}, \widehat{\theta}, \widehat{\gamma})$ with

$$
h_{i}(\widehat{\beta}, \widehat{\theta}, \widehat{\gamma})=\left(\sum_{k=1}^{K} \sum_{\ell=1}^{L^{(k)}} \widehat{\theta}_{k \ell} q_{k \ell}\left(W_{i}\right)\left(P_{i k}-W_{i}^{\prime} \widehat{\gamma}_{P_{k}}\right)-\widehat{\beta}\left(X_{i}-W_{i}^{\prime} \widehat{\gamma}_{X}\right)\right)\left(Z_{i}-W_{i}^{\prime} \widehat{\gamma}_{Z}\right)
$$

where $\widehat{\gamma}_{R}=\left(\sum_{i=1}^{N} W_{i} W_{i}^{\prime}\right)^{-1}\left(\sum_{i=1}^{N} W_{i} R_{i}\right)$ represents a regression coefficient of $R_{i}=X_{i}, Z_{i}, P_{i k}$ on $W_{i}$.

The moment conditions (A.7 A.8) jointly yield a stacked method-of-moments problem. Therefore, $\widehat{\beta}$ can be shown to be $\sqrt{N}$-consistent and asymptotically normal using the standard econometric results. In particular, under the regularity conditions in Newey and McFadden (1994, Section 6), the asymptotic expansion yields

$$
\begin{aligned}
& E\left[\frac{\partial}{\partial \beta} h_{i}(\beta, \theta, \gamma)\right] \sqrt{N}(\widehat{\beta}-\beta) \\
= & \frac{1}{\sqrt{N}} \sum_{i=1}^{N} h_{i}(\beta, \theta, \gamma)+E\left[\frac{\partial}{\partial \theta^{\prime}} h_{i}(\beta, \theta, \gamma)\right] E\left[B_{i} B_{i}^{\prime}\right]^{-1} \frac{1}{\sqrt{N}} \sum_{i=1}^{N} B_{i}\left(Y_{i}-B_{i}^{\prime} \theta\right) \\
& +E\left[\frac{\partial}{\partial \gamma^{\prime}} h_{i}(\beta, \theta, \gamma)\right] \sqrt{N}(\widehat{\gamma}-\gamma)+o_{P}(1) .
\end{aligned}
$$

Note that

$$
E\left[\frac{\partial}{\partial \gamma_{X}} h_{i}(\beta, \theta, \gamma)\right]=\beta E\left[W_{i} \widetilde{Z}_{i}\right]=0
$$

In addition, due to Assumption $\mathrm{L}_{-}$-(i),

$$
\begin{aligned}
E\left[\frac{\partial}{\partial \theta_{k \ell}} h_{i}(\beta, \theta, \gamma)\right] & =E\left[q_{k \ell}\left(W_{i}\right) \widetilde{P}_{i k} \widetilde{Z}_{i}\right]=E\left[q_{k \ell}\left(W_{i}\right) P_{i k} \widetilde{Z}_{i}\right], \\
E\left[\frac{\partial}{\partial \gamma_{P_{k}}} h_{i}(\beta, \theta, \gamma)\right] & =-E\left[W_{i} \widetilde{Z}_{i} \sum_{\ell=1}^{L^{(k)}} \theta_{k \ell} q_{k \ell}\left(W_{i}\right)\right]=0 .
\end{aligned}
$$

Finally,

$$
E\left[\frac{\partial}{\partial \gamma_{Z}} h_{i}(\beta, \theta, \gamma)\right]=-E\left[W_{i} \sum_{k=1}^{K} \alpha_{k}\left(W_{i}\right) \widetilde{P}_{i k}\right]
$$


Combining these results,

$$
\begin{aligned}
& E\left[\widetilde{X}_{i} \widetilde{Z}_{i}\right] \sqrt{N}(\widehat{\beta}-\beta) \\
= & \frac{1}{\sqrt{N}} \sum_{i=1}^{N}\left(Y_{2 i}-\beta \widetilde{X}_{i}\right) \widetilde{Z}_{i}+\frac{1}{\sqrt{N}} \sum_{i=1}^{N}\left(Y_{i}-B_{i}^{\prime} \theta\right) B_{i}^{\prime} E\left[B_{i} B_{i}^{\prime}\right]^{-1} E\left[B_{i} \widetilde{Z}_{i}\right] \\
& -\frac{1}{\sqrt{N}} \sum_{i=1}^{N} \widetilde{Z}_{i} W_{i}^{\prime} E\left[\widetilde{W}_{i} \widetilde{W}_{i}^{\prime}\right]^{-1} E\left[W_{i} Y_{2 i}\right] \\
= & \frac{1}{\sqrt{N}} \sum_{i=1}^{N}\left(\widetilde{Y}_{2 i}-\beta \widetilde{X}_{i}\right) \widetilde{Z}_{i}+\frac{1}{\sqrt{N}} \sum_{i=1}^{N}\left(Y_{i}-B_{i}^{\prime} \theta\right) B_{i}^{\prime} E\left[B_{i} B_{i}^{\prime}\right]^{-1} E\left[B_{i} \widetilde{Z}_{i}\right]+o_{P}(1),
\end{aligned}
$$

where $Y_{2 i}=\sum_{k=1}^{K} \alpha_{k}\left(W_{i}\right) \widetilde{P}_{i k}$. This completes the derivation of (27).

With a DID- or RD-type instrument, (A.8) is modified as

$$
h_{i}(\widehat{\beta}, \widehat{\theta}, \widehat{\gamma})=\left(\sum_{k=1}^{K}\left(\sum_{\ell=1}^{L^{(k)}} \widehat{\theta}_{k \ell} W_{i}^{\prime} \widehat{\gamma}_{q_{k \ell}}\right) P_{i k}-\widehat{\beta}\left(X_{i}-W_{i}^{\prime} \widehat{\gamma}_{X}\right)\right)\left(Z_{i}-W_{i}^{\prime} \widehat{\gamma}_{Z}\right),
$$

where $\widehat{\gamma}_{q_{k \ell}}=\left(\sum_{i=1}^{N} W_{i} W_{i}^{\prime}\right)^{-1}\left(\sum_{i=1}^{N} W_{i} q_{k \ell}\left(W_{i}\right)\right)$. For each $k=1, \ldots, K$,

$$
\begin{aligned}
& \sum_{\ell=1}^{L^{(k)}} E\left[\frac{\partial}{\partial \gamma_{q_{k \ell}}} h_{i}(\beta, \theta, \gamma)\right] \sqrt{N}\left(\widehat{\gamma}_{q_{k \ell}}-\gamma_{q_{k \ell}}\right) \\
= & \sum_{\ell=1}^{L^{(k)}} \theta_{k \ell} E\left[P_{i k} W_{i} \widetilde{Z}_{i}\right] E\left[W_{i} W_{i}^{\prime}\right]^{-1} \frac{1}{\sqrt{N}} \sum_{i=1}^{N} W_{i}\left(q_{k \ell}\left(W_{i}\right)-W_{i}^{\prime} \gamma_{q_{k \ell}}\right) \\
= & \frac{1}{\sqrt{N}} \sum_{i=1}^{N} L_{W_{i}}\left(P_{i k} \widetilde{Z}_{i}\right)\left(\alpha_{k}\left(W_{i}\right)-L_{W_{i}}\left(\alpha_{k}\left(W_{i}\right)\right)\right) .
\end{aligned}
$$

Then, the asymptotic expansion (A.10) should be modified as

$$
\begin{aligned}
& E\left[\widetilde{X}_{i} \widetilde{Z}_{i}\right] \sqrt{N}(\widehat{\beta}-\beta) \\
= & \frac{1}{\sqrt{N}} \sum_{i=1}^{N}\left(\widetilde{Y}_{2 i}-\beta \widetilde{X}_{i}\right) \widetilde{Z}_{i}+\frac{1}{\sqrt{N}} \sum_{i=1}^{N}\left(Y_{i}-B_{i}^{\prime} \theta\right) B_{i}^{\prime} E\left[B_{i} B_{i}^{\prime}\right]^{-1} E\left[B_{i} \widetilde{Z}_{i}\right] \\
& +\frac{1}{\sqrt{N}} \sum_{i=1}^{N} \sum_{k=1}^{K} L_{W_{i}}\left(P_{i k} \widetilde{Z}_{i}\right)\left(\alpha_{k}\left(W_{i}\right)-L_{W_{i}}\left(\alpha_{k}\left(W_{i}\right)\right)\right)+o_{P}(1),
\end{aligned}
$$

where $Y_{2 i}=\sum_{k=1}^{K} L_{W_{i}}\left(\alpha_{k}\left(W_{i}\right)\right) P_{i k}$ and $B_{i}^{\prime}=\left(L_{W_{i}}\left(q_{k 1}\left(W_{i}\right)\right) P_{i k}, \ldots, L_{W_{i}}\left(q_{k L^{(k)}}\left(W_{i}\right)\right) P_{i k}\right)_{k=1}^{K}$. 
Note that if $\alpha_{k}\left(W_{i}\right)$ is restricted to be linear, this expression is identical to (A.10) except that $Y_{2 i}$ is differently defined.

\section{C.5 Statistical Properties of the Generalized DWH Test Statistics}

Let $\left(Y_{i}, X_{i}, Z_{i}, W_{i}\right)_{i=1}^{N}$ be an i.i.d. random sample that satisfies the set of assumptions in Section 2. The numerator of the test statistic (28) defined in Section 4.3 can be expressed as

$$
\widehat{\tau}=\frac{1}{N} \sum_{i=1}^{N} h_{i}(\widehat{\theta}, \widehat{\gamma})
$$

where

$$
h_{i}(\widehat{\theta}, \widehat{\gamma})=\left\{\left(Y_{i}-W_{i}^{\prime} \widehat{\gamma}_{Y}\right)-\sum_{k=1}^{K}\left(P_{i k}-W_{i}^{\prime} \widehat{\gamma}_{P_{k}}\right) \sum_{\ell=1}^{L^{(k)}} \widehat{\theta}_{k \ell} q_{k \ell}\left(W_{i}\right)\right\}\left(Z_{i}-W_{i}^{\prime} \widehat{\gamma}_{Z}\right) .
$$

The population value of $\widehat{\tau}$ is given by

$$
\tau=E\left[h_{i}(\theta, \gamma)\right]=E\left[\left(\widetilde{Y}_{i}-Y_{2 i}\right) \widetilde{Z}_{i}\right]=E\left[\left(\widetilde{Y}_{i}-\widetilde{Y}_{2 i}\right) \widetilde{Z}_{i}\right]
$$

where $Y_{2 i}=\sum_{k=1}^{K} \alpha_{k}\left(W_{i}\right) \widetilde{P}_{i k}$.

To perform the asymptotic expansion, consider derivatives of $E\left[h_{i}(\theta, \gamma)\right]$. Due to Assumption $\amalg-(\mathrm{i})$,

$$
\begin{aligned}
& E\left[\frac{\partial}{\partial \gamma_{P_{k}}} h_{i}(\theta, \gamma)\right]=E\left[W_{i} \widetilde{Z}_{i} \sum_{\ell=1}^{L^{(k)}} \theta_{k \ell} q_{k \ell}\left(W_{i}\right)\right]=0 \\
& E\left[\frac{\partial}{\partial \theta_{k \ell}} h_{i}(\theta, \gamma)\right]=E\left[q_{k \ell}\left(W_{i}\right) \widetilde{P}_{i k} \widetilde{Z}_{i}\right]=E\left[q_{k \ell}\left(W_{i}\right) P_{i k} \widetilde{Z}_{i}\right] .
\end{aligned}
$$

In addition,

$$
\begin{aligned}
& E\left[\frac{\partial}{\partial \gamma_{Z}} h_{i}(\theta, \gamma)\right]=E\left[W_{i} Y_{2 i}\right], \\
& E\left[\frac{\partial}{\partial \gamma_{Y}} h_{i}(\theta, \gamma)\right]=0 .
\end{aligned}
$$

Combining these, the standard asymptotic formula in Newey and McFadden (1994, Section 
6) yields

$$
\begin{aligned}
\sqrt{N}(\widehat{\tau}-\tau) & =\frac{1}{\sqrt{N}} \sum_{i=1}^{N}\left\{\left(\widetilde{Y}_{i}-Y_{2 i}\right) \widetilde{Z}_{i}-E\left[\left(\widetilde{Y}_{i}-\widetilde{Y}_{2 i}\right) \widetilde{Z}_{i}\right]\right\} \\
& -\frac{1}{\sqrt{N}} \sum_{i=1}^{N}\left(Y_{i}-B_{i}^{\prime} \theta\right) B_{i}^{\prime} E\left[B_{i} B_{i}^{\prime}\right]^{-1} E\left[B_{i} \widetilde{Z}_{i}\right] \\
& +\frac{1}{\sqrt{N}} \sum_{i=1}^{N} \widetilde{Z}_{i} W_{i}^{\prime} E\left[W_{i} W_{i}\right]^{-1} E\left[W_{i} Y_{2 i}\right]+o_{P}(1) \\
= & \frac{1}{\sqrt{N}} \sum_{i=1}^{N}\left(\left(\widetilde{Y}_{i}-\widetilde{Y}_{2 i}\right) \widetilde{Z}_{i}-E\left[\left(\widetilde{Y}_{i}-\widetilde{Y}_{2 i}\right) \widetilde{Z}_{i}\right]-v_{1 i} \widehat{\widetilde{Z}}_{i}\right)+o_{P}(1)
\end{aligned}
$$

where $v_{1 i}=Y_{i}-B_{i}^{\prime} \theta$, and $\widehat{\widetilde{Z}}_{i}=B_{i}^{\prime} E\left[B_{i} B_{i}^{\prime}\right]^{-1} E\left[B_{i} \widetilde{Z}_{i}\right]$. Then, the central limit theorem implies $\sqrt{N}(\widehat{\tau}-\tau) \stackrel{d}{\rightarrow} N(0, \Sigma)$, where

$$
\Sigma=E\left[\left(\left(\widetilde{Y}_{i}-\widetilde{Y}_{2 i}\right) \widetilde{Z}_{i}-E\left[\left(\widetilde{Y}_{i}-\widetilde{Y}_{2 i}\right) \widetilde{Z}_{i}\right]-v_{1 i} \widehat{\widetilde{Z}}_{i}\right)^{2}\right]
$$

In addition,

$$
N \widehat{S}^{2}=\frac{1}{N} \sum_{i=1}^{N}\left(\left(\widetilde{Y}_{i}-\widetilde{Y}_{2 i}\right) \widetilde{Z}_{i}-\frac{1}{N} \sum_{j=1}^{N}\left(\widetilde{Y}_{j}-\widetilde{Y}_{2 j}\right) \widetilde{Z}_{j}-v_{1 i} \widehat{\widetilde{Z}}_{i}\right)^{2} \stackrel{p}{\rightarrow} \Sigma
$$

Therefore, the test statistic $\widehat{T}=\widehat{\tau} / \widehat{S}$ converges in distribution to $N(0,1)$ if $\tau=0$ and diverges if $\tau \neq 0$. Since $\tau=E\left[\widetilde{X}_{i} \widetilde{Z}_{i}\right] \cdot \Delta_{M E}$ and $E\left[\widetilde{X}_{i} \widetilde{Z}_{i}\right] \neq 0, \widehat{T}$ converges in distribution to $N(0,1)$ if $\Delta_{M E}=0$ and diverges if $\Delta_{M E} \neq 0.49$

With a DID- or RD-type instrument, (A.11) is modified as

$$
h_{i}(\widehat{\theta}, \widehat{\gamma})=\left\{\left(Y_{i}-W_{i}^{\prime} \widehat{\gamma}_{Y}\right)-\sum_{k=1}^{K} P_{i k} \sum_{\ell=1}^{L^{(k)}} \widehat{\theta}_{k \ell} W_{i}^{\prime} \widehat{\gamma}_{q_{k \ell}}\right\}\left(Z_{i}-W_{i}^{\prime} \widehat{\gamma}_{Z}\right) .
$$

\footnotetext{
${ }^{49}$ Since a feasible estimator for $\widehat{S}$ would be constructed using $Y_{2 i}=\sum_{k=1}^{K} \widehat{\alpha}_{k}\left(W_{i}\right) \widetilde{P}_{i k}$ instead of $Y_{2 i}=$

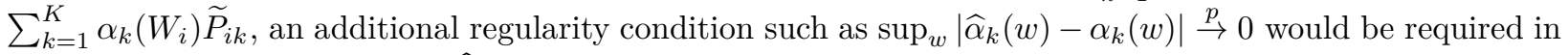
practice for the convergence $N \widehat{S}^{2} \stackrel{p}{\rightarrow} \Sigma$.
} 
For each $k=1, \ldots, K$,

$$
\begin{aligned}
& \sum_{\ell=1}^{L^{(k)}} E\left[\frac{\partial}{\partial \gamma_{q_{k \ell}}} h_{i}(\theta, \gamma)\right] \sqrt{N}\left(\widehat{\gamma}_{q_{k \ell}}-\gamma_{q_{k \ell}}\right) \\
= & -\sum_{\ell=1}^{L^{(k)}} \theta_{k \ell} E\left[P_{i k} W_{i} \widetilde{Z}_{i}\right] E\left[W_{i} W_{i}^{\prime}\right]^{-1} \frac{1}{\sqrt{N}} \sum_{i=1}^{N} W_{i}\left(q_{k \ell}\left(W_{i}\right)-W_{i}^{\prime} \gamma_{q_{k \ell}}\right) \\
= & -\frac{1}{\sqrt{N}} \sum_{i=1}^{N} L_{W_{i}}\left(P_{i k} \widetilde{Z}_{i}\right)\left(\alpha_{k}\left(W_{i}\right)-L_{W_{i}}\left(\alpha_{k}\left(W_{i}\right)\right)\right) .
\end{aligned}
$$

Then the asymptotic expansion in (A.12) is modified as

$$
\begin{aligned}
\sqrt{N}(\widehat{\tau}-\tau) & =\frac{1}{\sqrt{N}} \sum_{i=1}^{N}\left(\left(\widetilde{Y}_{i}-\widetilde{Y}_{2 i}\right) \widetilde{Z}_{i}-E\left[\left(\widetilde{Y}_{i}-\widetilde{Y}_{2 i}\right) \widetilde{Z}_{i}\right]-v_{1 i} \widehat{\widetilde{Z}}_{i}\right) \\
& -\frac{1}{\sqrt{N}} \sum_{i=1}^{N} \sum_{k=1}^{K} L_{W_{i}}\left(P_{i k} \widetilde{Z}_{i}\right)\left(\alpha_{k}\left(W_{i}\right)-L_{W_{i}}\left(\alpha_{k}\left(W_{i}\right)\right)\right)+o_{P}(1)
\end{aligned}
$$

where $Y_{2 i}=\sum_{\hat{~}=1}^{K} \alpha_{k}\left(W_{i}\right) P_{i k}, B_{i}^{\prime}=\left(L_{W_{i}}\left(q_{k 1}\left(W_{i}\right)\right) P_{i k}, \ldots, L_{W_{i}}\left(q_{k L^{(k)}}\left(W_{i}\right)\right) P_{i k}\right)_{k=1}^{K}, v_{1 i}=$ $Y_{i}-B_{i}^{\prime} \theta$, and $\widehat{\widetilde{Z}}_{i}=B_{i}^{\prime} E\left[B_{i} B_{i}^{\prime}\right]^{-1} E\left[B_{i} \widetilde{Z}_{i}\right]$. If $\alpha_{k}\left(W_{i}\right)$ is restricted to be linear, then this expression is identical to (A.12) except for the definition of $Y_{2 i}$.

\section{C.6 Monte Carlo Analysis}

This section provides a Monte Carlo analysis of the decomposition estimators and the generalized DWH test proposed proposed in the main paper. I consider a setting that closely follows a Monte Carlo analysis in Lochner and Moretti (2015), which originally draws on the schooling model in Card (1995). An individual $i$ chooses a schooling level $x \in\{0,1, \ldots, 20\}$ to maximize $Y_{i}(x)-C_{i}(x)$, where $Y_{i}(x)$ represents potential log earnings associated with the schooling level $x$ and $C_{i}(x)$ represents the cost to attain $x$ years of education. Each individual has an observed characteristics $W_{i}$ and a cost shifter $Z_{i}$. $W_{i}$ and $Z_{i}$ are mutually independent and each of them takes 0 or 1 with equal probability.

The potential earnings are given by

$$
Y_{i}(x)=b_{i} x+\kappa \mathbb{1}_{x \geq 12}+\varepsilon_{i}
$$

The parameter $\kappa$ represents the "sheepskin effect" of high school graduation. While Lochner and Moretti (2015) set the coefficient $b_{i}=0.04$ for all individuals, I allow it to be heteroge- 
neous. The schooling costs are given by

$$
C_{i}(x)=\eta_{i} x+d_{i} Z_{i}(x-12) \mathbb{1}_{x>12}+\frac{\gamma}{2} x^{2}+\kappa \mathbb{1}_{x \geq 12}+\left(b_{i}-E\left[b_{i}\right]\right) x .
$$

Following Lochner and Moretti (2015), I include $\kappa \mathbb{1}_{x \geq 12}$ in the cost function to ensure that the nonlinearity of the earnings function does not influence the schooling decision. In a similar spirit, I include $\left(b_{i}-E\left[b_{i}\right]\right) x$ to ensure that the schooling decision does not vary across different degrees of heterogeneity in $b_{i}$. This specification allows the exploration of different scenarios without changing the distribution of schooling and the IV and OLS weights. The coefficient $d_{i}$ represents the sensitivity of the schooling cost to the cost shifter $Z_{i}$. While Lochner and Moretti (2015) set $d_{i}=0.01$ for everyone and assume that the cost shifter $Z_{i}$ influences all schooling margins, I allow $d_{i}$ to be heterogeneous and assume that the cost shifter affects college education margins $(x>12)$ only. $\left(\varepsilon_{i}, \eta_{i}\right)$ follows a bivariate normal distribution independently from $\left(W_{i}, Z_{i}\right)$, with zero means and a variance matrix

$$
\left(\begin{array}{cc}
\sigma_{\varepsilon}^{2} & \rho_{\varepsilon \eta} \sigma_{\varepsilon} \sigma_{\eta} \\
\rho_{\varepsilon \eta} & \sigma_{\eta}^{2}
\end{array}\right)
$$

Since $\rho_{\varepsilon \eta}$ governs the correlation between the potential earnings and the schooling cost, $\rho_{\varepsilon \eta} \neq 0$ gives rise to endogeneity bias of the OLS coefficient. Conditional on $W_{i},\left(b_{i}, d_{i}\right)$ is independent from $\left(Z_{i}, \varepsilon_{i}, \eta_{i}\right)$ and follows a bivariate normal distribution

$$
\left[\begin{array}{c}
b_{i} \\
\ln d_{i}
\end{array}\right] \mid W_{i} \sim \operatorname{Normal}\left(\left[\begin{array}{c}
\mu_{b}+\delta_{b}\left(W_{i}-\frac{1}{2}\right) \\
\ln \mu_{d}+\delta_{d}\left(W_{i}-\frac{1}{2}\right)
\end{array}\right],\left[\begin{array}{cc}
\sigma_{b}^{2} & \rho_{b d} \sigma_{b} \sigma_{d} \\
\rho_{b d} \sigma_{b} \sigma_{d} & \sigma_{d}^{2}
\end{array}\right]\right)
$$

where $d_{i}$ is log-transformed to ensure a positive response to the cost shifter.

Following Lochner and Moretti (2015), I set $\mu_{b}=0.04, \mu_{d}=0.01, \gamma=0.003, \sigma_{\varepsilon}=0.5$, and $\sigma_{\eta}=0.0150$ In addition, I set $\delta_{d}=1$ and $\sigma_{d}=0.5$ to introduce observed and unobserved heterogeneity in the sensitivity coefficient $d_{i}$. Given the cost function specification, the IV weights are expected to be higher for a group with $W_{i}=1$ and college education margins $(x>12)$. For other parameters, I consider five cases below.

Case 1: The earnings function is linear: $\kappa=0$. The schooling coefficient $b_{i}$ is homogeneous in both observed and unobserved dimensions: $\left(\delta_{b}, \sigma_{b}\right)=(0,0)$.

Case 2: The earnings function is nonlinear: $\kappa=0.1$. The schooling coefficient $b_{i}$ is homo-

\footnotetext{
${ }^{50}$ Since the cost function specification slightly differs from Lochner and Moretti (2015), I set the parameter $\sigma_{\eta}$ higher than $\sigma_{\eta}=0.007$ in Lochner and Moretti (2015) to retain a reasonable schooling distribution. Years of schooling in the simulated sample has the mean 12.6 and the standard deviation 2.9, which are comparable to the values in the 1980 U.S. Census data.
} 
geneous in both observed and unobserved dimensions: $\left(\delta_{b}, \sigma_{b}\right)=(0,0)$.

Case 3: The earnings function is nonlinear: $\kappa=0.1$. The schooling coefficient $b_{i}$ is heterogeneous only in an observed dimension: $\left(\delta_{b}, \sigma_{b}\right)=(-0.04,0)$.

Case 4: The earnings function is nonlinear: $\kappa=0.1$. The schooling coefficient $b_{i}$ is heterogeneous in both observed and unobserved dimensions: $\left(\delta_{b}, \sigma_{b}\right)=(-0.04,0.02)$. However, these is no unobservable-driven correlation between $b_{i}$ and the sensitivity $d_{i}$ to the instrument: $\rho_{b d}=0$.

Case 5: The earnings function is nonlinear: $\kappa=0.1$. The schooling coefficient $b_{i}$ is heterogeneous in both observed and unobserved dimensions: $\left(\delta_{b}, \sigma_{b}\right)=(-0.04,0.02)$. These is unobservable-driven correlation between $b_{i}$ and $d_{i}: \rho_{b d}=-0.25$.

I explore three different levels of endogeneity, $\rho_{\varepsilon \eta} \in\{0,0.1,0.2\}$, for each of the five cases 51 $\kappa>0$ in Cases 2-5 implies that the marginal return to the 12 th year of education is higher than the marginal return to college education. Given that the instrument $Z_{i}$ influences college education costs, it is expected that the treatment-level weight difference pushes down the IV coefficient. $\delta_{b}<0$ in Cases 3-5 implies that individuals with $W_{i}=1$, who are more sensitive to the instrument, have smaller returns to schooling. Therefore, it is expected that the covariate weight difference pushes down the IV coefficient. $\rho_{b d}<0$ in Case 5 implies that there is an unobservable factor that increases sensitivity to the instrument and decreases returns to schooling. This is expected to further push down the IV coefficient by making the IV-identified marginal effect smaller than the AME.

For each possible combination of parameters, I perform 1,000 Monte Carlo simulations with the sample size $N=5,000$. In each simulation, I estimate the OLS and IV coefficients and the decomposition estimators. I perform three endogeneity tests below.

1. A standard DWH test, which examines the significance of the raw IV-OLS gap $\widehat{\beta}_{I V}-\widehat{\beta}_{O L S}$. I use the heteroskedasticity-robust standard errors unlike in the classic version of the test.

2. A generalized DWH test proposed by Lochner and Moretti (2015).

3. A generalized DWH test proposed in the main paper.

For expositional convenience, I refer to the first one as the standard DWH test, the second one as the DWH-LM test, and the third one as the DWH-I test.

\footnotetext{
${ }^{51} \rho_{\varepsilon \eta}>0$ gives rise to downward endogeneity bias of the OLS coefficient, which is opposite to the expected direction of endogeneity bias in the return-to-schooling context. Nevertheless, I consider a positive $\rho_{\varepsilon \eta}$ following the setting in Lochner and Moretti (2015).
} 
Table A.1 and A.2 report Monte Carlo simulation results. For each model, the tables report the means and the standard deviations of the point estimates of the OLS coefficient, the IV coefficient, the IV-OLS gap, and three components of the decomposition. In addition, the table reports the fraction of simulation draws in which each of the three endogeneity tests rejects the null at a $5 \%$ significance level. The fraction of rejection of an asymptotically unbiased test is expected to be close to $5 \%$ with $\rho_{\varepsilon \eta}=0$ and larger than $5 \%$ with $\rho_{\varepsilon \eta} \neq 0$.

Since Case 1 boils down to a classic linear model, all three versions of the DWH test are expected to be asymptotically unbiased. The first panel of Table A.1 confirms this expectation. The standard DWH test has a slightly smaller fraction of Type II errors than the other two tests. Even though the true earnings function is linear, endogeneity of schooling $X_{i}$ makes the conditional mean function $m(x, w)$ nonlinear. As a result, the DWH-LM test and the DWH-I test attribute a part of the endogeneity bias to the treatment-level weight difference. 52 On the other hand, I find no sizable difference in performance across the DWHLM test and the DWH-I test.

Case 2 corresponds to a model considered in Lochner and Moretti (2015). The true relationship between earnings and schooling is nonlinear but homogeneous. The second panel of Table A.1 confirms that the standard DWH test is no longer valid due to nonlinearity. On the other hand, the other two tests perform well, with similar fractions of Type I and Type II errors.

Case 3 corresponds to a separable model considered in Section 2 of the main paper. The true relationship between earnings and schooling is nonlinear and observably heterogeneous. While the DWH-I test is expected to work, the other two tests are expected to fail due to heterogeneity. In this setting, the marginal return to $x$ th year of education is $0.02 W_{i}+$ $0.06\left(1-W_{i}\right)+0.1 \mathbb{1}_{x=12}$ and the IV weights are concentrated on a group with $W_{i}=1$ and college education margins $(x>12)$. As a result, both the covariate weight difference and the treatment-level weight difference negatively contributes to the IV-OLS gap. As confirmed by the third panel of Table A.1, only the DWH-I test performs well as it successfully accounts for both types of the weight difference contributions.

Case 4 and Case 5 demonstrate that the decomposition framework in the main paper provides relevant information even when the separability assumption does not hold. Both of these cases fit into a nonseparable model considered in Section 3.2 of the main paper. The true relationship between earnings and schooling is nonlinear and heterogeneous in both observable and unobservable dimensions. Unobservable-driven correlation between the

\footnotetext{
${ }^{52}$ While the spurious nonlinearity of the conditional mean function works in favor of the standard DWH test and against the other two tests in this particular case, it can work in the opposite way depending on the IV weight patterns.
} 
treatment effect and the treatment sensitivity to the instrument is absent in Case 4 but present in Case 5. The DWH-I test is expected to still work in Case 4, since there is no discrepancy between the IV-identified marginal effect and the AME in this case. This expectation is confirmed by the first panel of Table A.2. On the other hand, the DWHI test is no longer valid in Case 5, as confirmed by the second panel of Table A.2. For example, even with $\rho_{\varepsilon \eta}=0$, the estimated marginal effect difference component $\widehat{\Delta}_{M E}$ is biased downward from 0 . This is because $\widehat{\Delta}_{M E}$ captures the negative gap between the IVidentified marginal effect and the AME, which arises from the negative correlation between $b_{i}$ and $d_{i}$. Nevertheless, $\widehat{\Delta}_{M E}$ is closer to zero than the raw IV-OLS gap, as it still accounts for the covariate weight difference and the treatment-level weight difference components.

\section{Empirical Details and Additional Results}

This section provides additional information and results for empirical applications in the main paper.

\section{D.1 College Cost Instrument with Geographic Variation}

Table A.3 reports the sample selection criteria for the analysis sample in Section 5.1 in detail. Most individuals have hourly wages reported at least once, which makes sample selection bias associated with labor force participation less of a concern.

Table A.4 presents the descriptive statistics of the analysis sample. As the wage measure, I use the log hourly wage at the current or most recent job, deflated using CPI-U (with the base year of 1982-84), bottom-coded at $\$ 1$, and top-coded at $\$ 100$. Years of education is based on the highest grade completed as of May 1 of the survey year (HGCREV), top-coded at 18. I use the age-adjusted Armed Forces Qualification Test (AFQT) percentile (AFQT-3) as the AFQT score measure. In the regressions, I use piecewise linear functions of AFQT and age with two kink points to ensure the flexible functional form. In particular, AFQT, $\max \left\{\mathrm{AFQT}-\frac{1}{3}, 0\right\}, \max \left\{\mathrm{AFQT}-\frac{2}{3}, 0\right\}$, age, $\max \{$ age $-35,0\}$, and $\max \{$ age $-45,0\}$ are included in a vector of covariates. Parental education dummies in the covariate vector follow the categories defined in Table A.4. Local earnings are based on the annual total earnings divided by the total employment, taken from the county-level economic accounts by the Bureau of Economic Analysis. Local unemployment rates are based on the state-level annual unemployment rates from the Bureau of Labor Statistics (BLS) 53 Infor-

\footnotetext{
${ }^{53}$ Because the BLS website only provides the data from 1976, I take the 1974-75 data from the 1977 Statistical Abstract of the United States.
} 
mation about public four-year colleges is based on the Higher Education General Information Survey (HEGIS) in 1977. Following Carneiro et al. (2011), I use the HEGIS data compiled by Kling (2001) to construct the college variables. The urban status of each county is based on whether the majority of the county's population lives in an urbanized area 54

Table A.5 reports the first-stage estimates. Local college availability and local tuition rate coefficients have the expected signs: smaller pecuniary or psychological costs of college attendance result in longer years of schooling. Local earnings and unemployment coefficients also have the expected signs: worse local labor market conditions reduce opportunity costs of college attendance and thus increase years of schooling.

Table A.6 explores the IV-OLS coefficient gap and its decomposition in alternative specifications. It is common across most specifications that the weight difference components positively contribute to the IV-OLS gap. However, it is notable that the weight difference contributions are estimated to be negative for the female sample and the sample of blacks and Hispanics.

Table A.7 explores the mechanisms underlying the difference in the decomposition results across different sample specifications. Panel (A) of the table shows the OLS weights on AFQT and parental education groups, high school margins of schooling, and college margins of schooling, for different samples. Panel (B) of the table presents the IV weights analogously. Panel (C) presents the OLS schooling coefficient given by a regression restricted to each covariate group or each schooling margin. I find no substantial difference in weight patterns across the male and female samples, although point estimates of the IV weights among males are somewhat more concentrated on advantaged groups and college education margins. The male sample has much larger disparity in OLS-identified returns to schooling across personal backgrounds and across schooling levels. Among blacks and Hispanics, the IV weights are not particularly concentrated on advantaged groups. In addition, their OLS-identified returns to schooling differs less across high school and college margins, compared with non-black, non-Hispanic individuals.

\section{D.2 RD-Based Compulsory Schooling Instrument}

In Section 5.2, I use the British General Household Survey (GHS) data provided by Oreopoulos (2008), which makes several corrections to the data used in Oreopoulos (2006) 55 Table A.8 presents the descriptive statistics from British and Northern Irish GHS data. While my

\footnotetext{
${ }^{54}$ Urbanized areas are defined by the U.S. Census Bureau based on the 1990 Census.

${ }^{55}$ In addition to the corrections made by Oreopoulos (2008), I correct a small number of observations with erratic reported ages. In particular, I compare the reported age with the difference between survey and birth years, and replace the former with the latter if two age measures differ by more than one year.
} 
main analysis focuses on the British GHS in due to its much larger sample size, Oreopoulos (2006) estimates the returns to schooling in Northern Ireland in addition to those in Britain.

Table A.9 explores several alternative specifications. The first row of the table reproduces the main result in Table 1. The second row presents the decomposition result with no topcoding of years schooling, following the original specification in Oreopoulos (2006). The third and fourth rows show the decomposition results using the Northern Irish GHS with and without top-coding of the schooling levels. Because Northern Ireland raised the minimum school-leaving age in 1957, the instrument is an indicator for 1943 or later birth cohorts, who turned age 14 in 1957 or later. All the other variables are defined in the same manner as in the British GHS. Across all four specifications, the weight difference components are estimated to negatively contribute to the IV-OLS coefficient gap.

Table A.10 presents the estimated weights on covariate groups in the Northern Irish GHS. The IV weights are concentrated on individuals turning age 14 around the reform year, which is 10 years later than the British sample. Because the cohorts influenced by the reform have similar levels of OLS-identified returns to schooling to the other cohorts, the weight difference across cohorts are expected to contribute little to the IV-OLS coefficient gap. This expectation is consistent with a small impact of the covariate weight difference in Table A.9.

Figure A.1 illustrates the patterns of OLS and IV weights on schooling levels. Unlike in the British sample, the IV weights are not entirely concentrated on the 10th year of schooling. While the IV weights are not precisely estimated due to a small sample size, it is possible that cohorts turning 14 around the reform year were exposed to other concurrent institutional or economic shocks to schooling levels.

\section{D.3 Compulsory Schooling Instrument with DID Variation}

I use the 1960-80 U.S. Census data compiled by IPUMS (Ruggles et al., 2020) in Section 5.3. Following Acemoglu and Angrist (2000), I use the 1\% sample from the 1960 Census, a pooled $2 \%$ sample from the 1970 Census by combining the 1\% State Form 1 and Form 2 samples, and the $5 \%$ sample from the 1980 Census. Due to the inconsistency of the sampling rates across Census years, I weight observations from the 1960 Census by 5 and observations from the 1970 Census by 2.5, relative to observations from the 1980 Census. I use the compulsory schooling laws (CSL) data provided by Acemoglu and Angrist (2000). Due to missing CSL status for Alaska and Hawaii in their data, I drop individuals born in these two states from the analysis sample.

Acemoglu and Angrist (2000) use the 1960-80 U.S. Census data in their main specifica- 
tion, which my main analysis follows. As they use the 1950 and 1990 Census data in some other specifications, they modify several variables in the 1960-80 Census data to maintain consistency across all Census years. I do not implement these modifications because they are unnecessary for analyzing the 1960-80 Census data alone. There are three main differences between my specification and theirs for this reason. First, I top-code years of schooling at 18 because the 1960-70 Censuses report years of schooling with a cap at 18 (the cap is 20 years in the 1980 Census). On the other hand, Acemoglu and Angrist (2000) use 17 years as a common cap to the 1950 Census. Second, Acemoglu and Angrist (2000) impute year of birth, which is unavailable in the 1950 Census data, from age as of the Census day and quarter of birth even in other Census years. I do not follow this approach because year of birth is always available in the 1960-80 Census data. Finally, in all Census years, they determine the CSL status from age as of Census day and state of birth. I determine the CSL status from year of birth and state of birth to ensure that individuals in the same cohort and the same state are assigned with the same CSL status.

Table A.11 presents descriptive statistics for each Census year. I compute weekly earnings by dividing wage and salary income by weeks worked in the previous year. Because weeks worked are given in intervals in the 1960-70 Censuses, I use group means in the 1980 Census to represent the intervals. I bottom-code weekly earnings at $\$ 20$ for the 1960 sample, at $\$ 26$ for the 1970 sample, and at $\$ 58$ for the 1980 sample, which respectively correspond to 20 times the federal minimum wage in 1959, 1969, and 1979. Income is reported with a top-coding at $\$ 25,000, \$ 50,000$, and $\$ 75,000$ in each decennial Census. Thus, I impose a top-coding on weekly earnings at $\$ 25,000 / 52, \$ 50,000 / 52$, and $\$ 75,000 / 52$ for each Census year. Table A.12 provides the first-stage coefficients of the CSL instruments. Patterns of the estimated coefficients are consistent with the expectation that more stringent CSL requirements result in longer years of schooling.

Table A.13 presents the decomposition results with alternative compulsory schooling instruments. The first row of the table reproduces the main result in Table 1 that uses required years of schooling implied by child labor laws. The second row presents the result with required years of schooling implied by compulsory attendance laws 56 The third row shows the result with required schooling years jointly implied by child labor and compulsory attendance laws, as compiled by Stephens and Yang (2014). The fourth row shows the result using quarter-of-birth dummies interacted with dummies for the Census years as the instruments. The weight difference components positively contribute to the IV-OLS gap in the decompo-

\footnotetext{
${ }^{56}$ The definition of compulsory attendance laws follows Acemoglu and Angrist (2000). In computing the required number of years, I use the corrected formula suggested by Goldin and Katz (2011) and Stephens and Yang (2014), which replaces the max function in the original formula in Acemoglu and Angrist (2000) with the min function.
} 
sition results with compulsory attendance laws or required schooling years, similarly to the result with child labor laws. On the other hand, with quarter-of-birth instruments, the IV and OLS estimates are nearly identical and the weight difference components are close to zero.

Table A.14 shows the estimated OLS and IV weights on covariate groups. The patterns of the compulsory attendance and required schooling IV weights are similar to that of the child labor IV weights presented in Table 4 in the main paper. On the other hand, the quarter-of-birth IV weights do not substantially differ from the OLS weights.

The estimated OLS and IV weights on treatment levels are illustrated in Figure A.2. The patterns of the IV weights associated with compulsory attendance laws or required schooling years are close to those of the child labor IV weights illustrated in Panel (c) of Figure 1 in the main paper. On the other hand, the quarter-of-birth IV weights are somewhat closer to the OLS weights, although the difference is still visible. In fact, $55 \%$ of the OLS weights are placed on the 1st-12th years of schooling, while $74 \%$ of the quarter-of-birth IV weights are placed on the same schooling margins.

Higher quarter-of-birth IV weights on primary and secondary schooling margins are consistent with the expectation that the season of birth should influence schooling because of the age-based CSL. However, it should be noted that the non-negligible share of IV weights is placed on college education years. While it is possible that students who are forced to complete an additional year of schooling may proceed with further schooling, the weight patterns associated with the other two compulsory schooling instruments in Section 5 do not indicate such dynamic effects. Therefore, this evidence is rather consistent with the argument of Bound and Jaeger (2000) that the influence of the season of birth on schooling is too strong to be fully explained by the CSL. It is likely that the season of birth influences schooling through other factors, such as the persistence of a relative age effect during childhood 57

\section{D.4 Empirical Specification of the Decomposition Estimators}

I use the two-step method proposed in Section 4.1 to obtain the IV-weighted OLS estimates. I use the parametric specifications

$$
m(x, w)=w^{\prime} \gamma_{1}+\left(w^{\prime} \gamma_{2}\right) x
$$

\footnotetext{
${ }^{57}$ In fact, Kawaguchi (2011) finds that early-born children in a given cohort tend to complete more years of schooling in Japan, where the CSL are cohort-based instead of age-based.
} 
for estimating $\beta_{C}$ and

$$
m(x, w)=w^{\prime} \gamma_{1}+\left(w^{\prime} \gamma_{2}\right) x+\left(w^{\prime} \gamma_{3}\right) \max \{x-\bar{x}, 0\}+\sum_{x} \delta_{x} \mathbb{1}_{X \geq x}
$$

for estimating $\beta_{C T} . \bar{x}$ is a constant that represents the last year of secondary school, where $\bar{x}=12$ in Sections 5.1 and 5.3 and $\bar{x}=11$ in Section 5.2. Interacting $\max \{x-\bar{x}, 0\}$ with $w$ allows return to schooling at post-secondary schooling margins to have different patterns of heterogeneity from primary and secondary schooling margins. Including $\mathbb{1}_{X \geq x}$ in the equation allows arbitrary nonlinearity of $m(x, w)$ in $x$. Since the covariate vector $w$ in Section 5.3 consists of a large number of variables (i.e., year of birth and state of birth dummies), I restrict $w^{\prime} \gamma_{1}, w^{\prime} \gamma_{2}$, and $w^{\prime} \gamma_{3}$ to be additive in 5-year cohort group dummies and Census division dummies to avoid overstating heterogeneity.

I estimate the OLS and IV covariate weights and treatment-level weights from the data counterparts of how they are defined in Section 2.2 and 3.1. I estimate the OLS and IV weight on treatment level $x, \bar{\omega}_{X}(x)$ and $\bar{\omega}_{Z}(x)$ (or $\bar{\omega}_{Z}^{*}(x)$ equivalently), from

$$
\begin{aligned}
& \frac{\sum_{i=1}^{N} \widetilde{\mathbb{1}}_{X_{i} \geq x} \widetilde{X}_{i}}{\sum_{i=1}^{N} \widetilde{X}_{i}^{2}}, \\
& \frac{\sum_{i=1}^{N} \widetilde{\mathbb{1}}_{X_{i} \geq x} \widetilde{Z}_{i}}{\sum_{i=1}^{N} \widetilde{X}_{i} \widetilde{Z}_{i}} .
\end{aligned}
$$

I estimate the total OLS weight on covariate group $G, \int_{w \in G} \bar{\omega}_{X}(w) d F_{W}(w)$, from

$$
\frac{\sum_{i=1}^{N} \mathbb{1}_{W_{i} \in G} \tilde{X}_{i}^{2}}{\sum_{i=1}^{N} \widetilde{X}_{i}^{2}}
$$

In Section 5.1, I estimate the total IV weight on covariate group $G, \int_{w \in G} \bar{\omega}_{Z}(w) d F_{W}(w)$, from

$$
\frac{\sum_{i=1}^{N} \mathbb{1}_{W_{i} \in G} \widetilde{X}_{i} \widetilde{Z}_{i}}{\sum_{i=1}^{N} \widetilde{X}_{i} \widetilde{Z}_{i}},
$$

In Sections 5.2 and 5.3, which use RD-based and DID-based instruments, I estimate the IV weight on covariate group $G, \int_{w \in G} \bar{w}_{Z}^{*}(w) d F_{W}(w)$, from

$$
\frac{\sum_{i=1}^{N} \mathbb{1}_{W_{i} \in G} \widehat{L}_{W_{i}}\left(X_{i} \widetilde{Z}_{i}\right)}{\sum_{i=1}^{N} \widetilde{X}_{i} \widetilde{Z}_{i}}
$$

where $\widehat{L}_{W_{i}}\left(X_{i} \widetilde{Z}_{i}\right)=W_{i}^{\prime}\left(\sum_{j=1}^{N} W_{j} W_{j}^{\prime}\right)^{-1}\left(\sum_{j=1}^{N} W_{j} X_{j} \widetilde{Z}_{j}\right)$ 


\section{Appendix References}

Acemoglu, Daron and Joshua Angrist (2000). "How large are human-capital externalities? Evidence from compulsory schooling laws". NBER Macroeconomics Annual 15, pp. 9-59.

Ackerberg, Daniel, Xiaohong Chen, and Jinyong Hahn (2012). "A practical asymptotic variance estimator for two-step semiparametric estimators". Review of Economics and Statistics 94.2, pp. 481-498.

Angrist, Joshua D, Kathryn Graddy, and Guido W Imbens (2000). "The interpretation of instrumental variables estimators in simultaneous equations models with an application to the demand for fish". The Review of Economic Studies 67.3, pp. 499-527.

Angrist, Joshua D and Guido W Imbens (1995). "Two-stage least squares estimation of average causal effects in models with variable treatment intensity". Journal of the American Statistical Association 90.430, pp. 431-442.

Borusyak, Kirill, Xavier Jaravel, and Jann Spiess (2021). "Revisiting Event Study Designs: Robust and Efficient Estimation". Working Paper.

Bound, John and David A Jaeger (2000). "Do compulsory school attendance laws alone explain the association between quarter of birth and earnings?" Research in labor economics 19.4, pp. 83-108.

Card, David (1995). "Using Geographic Variation in College Proximity to Estimate the Return to Schooling". In: Toronto: University of Toronto Press.

Carneiro, Pedro, James J Heckman, and Edward J Vytlacil (2011). "Estimating marginal returns to education". American Economic Review 101.6, pp. 2754-81.

de Chaisemartin, Clément and Xavier D'Haultfoeuille (2020). "Two-Way Fixed Effects Estimators with Heterogeneous Treatment Effects." American Economic Review 110.9, pp. 2964-2996.

Goldin, Claudia and Lawrence F Katz (2011). "Mass Secondary Schooling and the State: The Role of State Compulsion in the High School Movement". In: University of Chicago Press, pp. 275-310.

Heckman, James J, Sergio Urzua, and Edward Vytlacil (2006). "Understanding instrumental variables in models with essential heterogeneity". The Review of Economics and Statistics 88.3, pp. 389-432.

Imbens, Guido W and Joshua D Angrist (1994). "Identification and estimation of local average treatment effects". Econometrica 62.2, pp. 467-475. 
Kawaguchi, Daiji (2011). "Actual age at school entry, educational outcomes, and earnings". Journal of the Japanese and International Economies 25.2, pp. 64-80.

Kling, Jeffrey R (2001). "Interpreting instrumental variables estimates of the returns to schooling". Journal of Business 65 Economic Statistics 19.3, pp. 358-364.

Lochner, Lance and Enrico Moretti (2015). "Estimating and testing models with many treatment levels and limited instruments". Review of Economics and Statistics 97.2, pp. 387397.

Montiel Olea, José Luis and Carolin Pflueger (2013). "A robust test for weak instruments". Journal of Business \& Economic Statistics 31.3, pp. 358-369.

Newey, Whitney K and Daniel McFadden (1994). "Large sample estimation and hypothesis testing". Handbook of Econometrics 4, pp. 2111-2245.

Oreopoulos, Philip (2006). "Estimating average and local average treatment effects of education when compulsory schooling laws really matter". American Economic Review 96.1, pp. $152-175$.

- (2008). "Estimating average and local average treatment effects of education when compulsory schooling laws really matter: Corrigendum". American Economic Review.

Ruggles, Steven et al. (2020). "IPUMS USA: Version 10.0 [dataset]". Minneapolis, MN: IPUMS. URL: https://doi.org/10.18128/D010.V10.0.

Schechtman, E and S Yitzhaki (2004). "The Gini Instrumental Variable, or the "double instrumental variable" estimator". Metron, pp. 287-313.

Stephens Melvin, Jr. and Dou-Yan Yang (2014). "Compulsory Education and the Benefits of Schooling". American Economic Review 104.6, pp. 1777-92.

Yitzhaki, Shlomo (1996). "On using linear regressions in welfare economics". Journal of Business 8 Economic Statistics 14.4, pp. 478-486. 


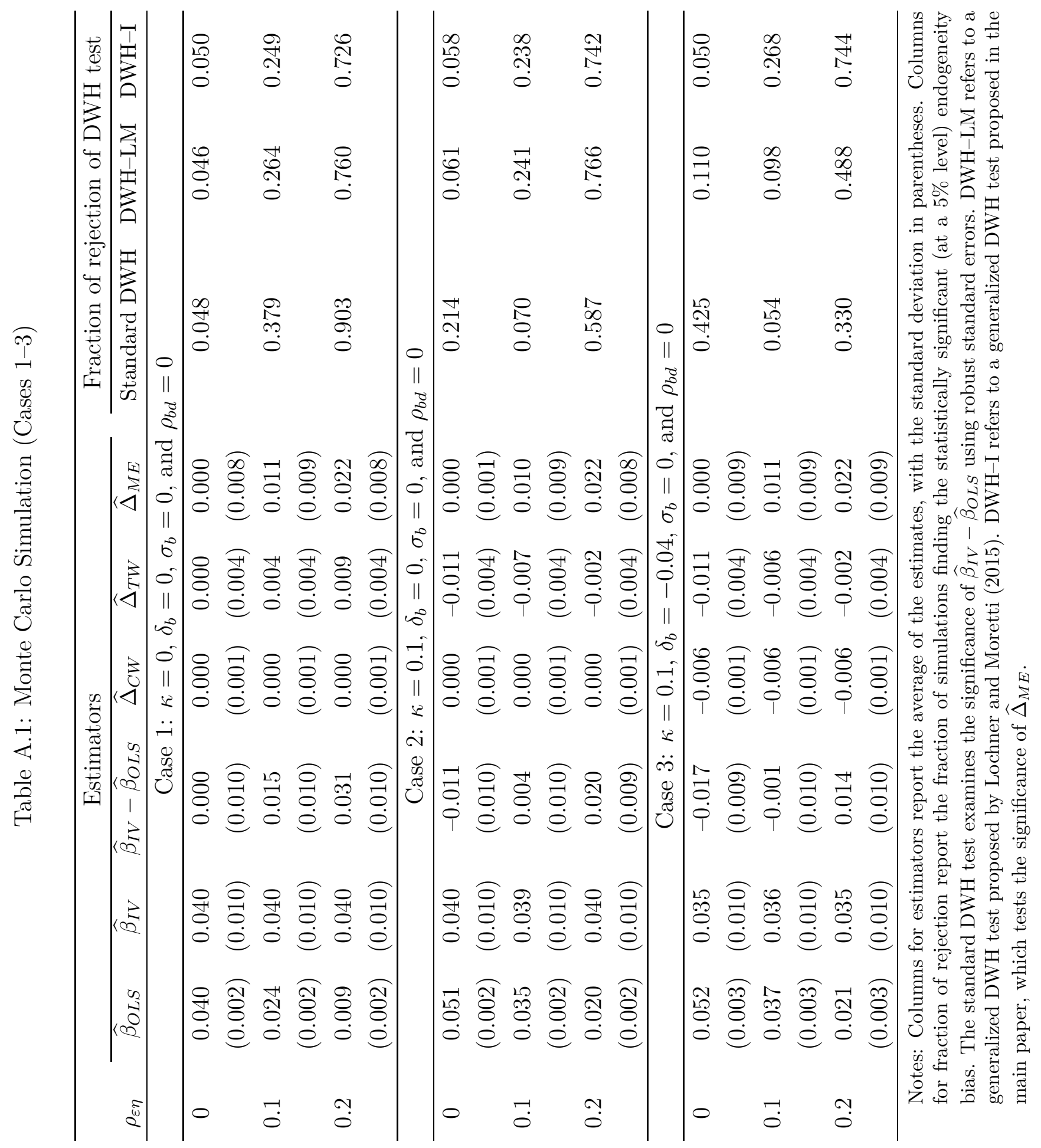




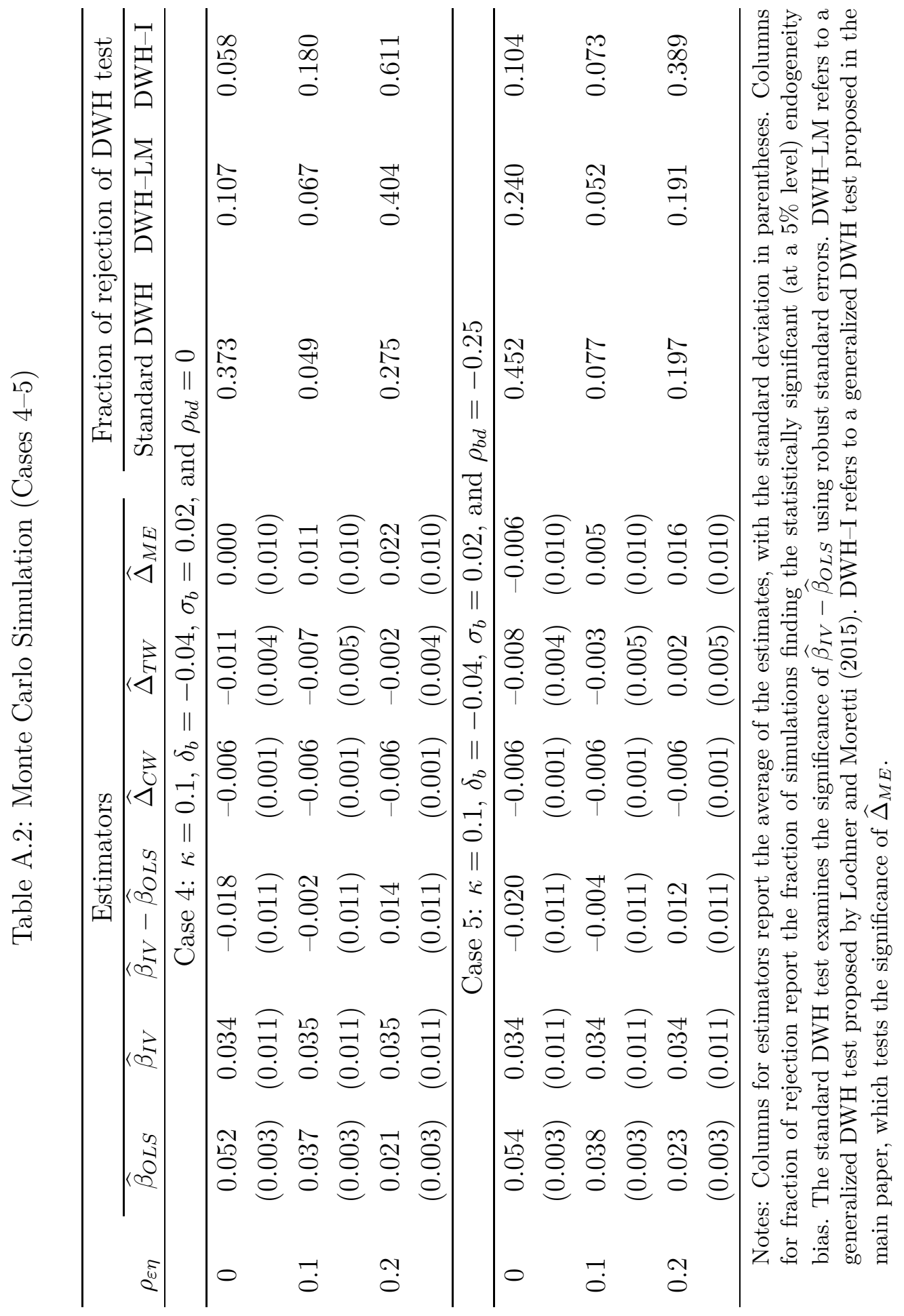


Table A.3: NLSY79 Sample Selection

\begin{tabular}{|c|c|c|c|c|}
\hline & \multicolumn{2}{|c|}{ Males } & \multicolumn{2}{|c|}{ Females } \\
\hline & Persons & Person-Years & Persons & Person-Years \\
\hline Civilian Sample & 5,579 & & 5,827 & \\
\hline \multicolumn{5}{|l|}{ Restrictions to Persons } \\
\hline AFQT available & -343 & & -307 & \\
\hline Age-14 location available & -273 & & -265 & \\
\hline 8th grade until age 22 & -60 & & -72 & \\
\hline Subtotal & 4,903 & 107,137 & 5,183 & 115,684 \\
\hline \multicolumn{5}{|c|}{ Restrictions to Person-Year Observations } \\
\hline Between age 25 and 54 & -93 & $-35,108$ & -62 & $-36,655$ \\
\hline Not currently enrolled & -15 & $-2,948$ & -4 & $-4,351$ \\
\hline Wage observed & -76 & $-8,569$ & -131 & $-15,589$ \\
\hline Subtotal & 4,719 & 60,512 & 4,986 & 59,089 \\
\hline
\end{tabular}

Notes: Negative values refer to the number of persons or observations dropped from the sample. Positive values indicate the number of persons or observations remaining in the sample. 
Table A.4: NLSY79 Descriptive Statistics

\begin{tabular}{lcc}
\hline & Mean & (S.D.) \\
\hline Variables by Person-Years & & \\
Hourly wage (\$) & 9.56 & $(10.31)$ \\
Years of schooling & 13.41 & $(2.30)$ \\
Age & 35.56 & $(8.57)$ \\
\hline Personal Background Variables & & \\
AFQT percentile & 0.506 & $(0.286)$ \\
Number of siblings & 3.07 & $(1.71)$ \\
Female & 0.504 & \\
Black & 0.141 & \\
Hispanic & 0.055 & \\
\hline Geographic Background Variables (age-14 county) & \\
Local earnings in 1974-81 (\$1,000) & 19.44 & $(3.55)$ \\
Unemployment rate in 1974-81 (\%) & 7.05 & $(1.27)$ \\
Local earnings at age 17 (\$1,000) & 19.48 & $(3.74)$ \\
Unemployment rate at age 17 (\%) & 7.04 & $(1.85)$ \\
Tuition rate of public 4-year (\$1,000) & 1.09 & $(0.40)$ \\
Urban status & 0.689 & \\
Presence of public 4-year & 0.666 & \\
\hline Parental Education & Father & Mother \\
Missing & 0.096 & 0.048 \\
Less than high school & 0.148 & 0.102 \\
Some high school & 0.149 & 0.190 \\
High school graduate (no college) & 0.332 & 0.452 \\
Some college & 0.103 & 0.111 \\
College graduate & 0.173 & 0.097 \\
\hline
\end{tabular}

Notes: Persons are weighted based on the sampling weights. Personyear observations are equally weighted within each person. Standard deviations are in parentheses for nonbinary variables. The number of siblings is top-coded at 6 . Hourly wage is bottom-coded at $\$ 1$ and top-coded at $\$ 100$. Hourly wage, local earnings, and tuition rate are deflated using CPI-U with the base year of 1982-84. 
Table A.5: First-Stage Coefficients of the College Cost Instruments

\begin{tabular}{lcc}
\hline & Coef. & S.E. \\
\hline Geographic Costs of Attendance & 0.080 & $(0.059)$ \\
Public 4-year present & -0.148 & $(0.087)$ \\
Tuition rate of public 4-year $(\$ 1,000)$ & & \\
Opportunity Costs of Attendance & -0.886 & $(0.475)$ \\
$\quad$ Log local earnings at age 17 & 0.021 & $(0.022)$ \\
$\quad$ Unemployment rate at age $17(\%)$ & \multicolumn{2}{c}{2.64} \\
\hline Effective F-stat.
\end{tabular}

Notes: Standard errors (in parentheses) are robust to heteroskedasticity and correlation across observations on persons living in the same county at age 14. The effective F-statistics is based on Montiel Olea and Pflueger (2013). 
Table A.6: Alternative Specifications for Decomposing the IV-OLS Coefficient Gap (NLSY79)

\begin{tabular}{|c|c|c|c|c|c|c|c|c|}
\hline & \multicolumn{3}{|c|}{ Coefficients } & \multicolumn{3}{|c|}{ Decomposition } & \multirow{2}{*}{$\begin{array}{c}\text { No. of } \\
\text { Obs. }\end{array}$} \\
\hline & & OLS & IV & IV-OLS & $\overline{\Delta_{C W}}$ & $\overline{\Delta_{T W}}$ & $\Delta_{M E}$ & \\
\hline & Base specification & $\begin{array}{c}0.065 \\
(0.003)\end{array}$ & $\begin{array}{c}0.062 \\
(0.087)\end{array}$ & $\begin{array}{l}-0.004 \\
(0.087)\end{array}$ & $\begin{array}{c}0.011 \\
(0.011)\end{array}$ & $\begin{array}{c}0.018 \\
(0.010)\end{array}$ & $\begin{array}{l}-0.032 \\
(0.086)\end{array}$ & 119,601 \\
\hline 2. & $\begin{array}{l}\text { Cross-sectional } \\
\text { sample }^{\mathrm{a}}\end{array}$ & $\begin{array}{c}0.065 \\
(0.004)\end{array}$ & $\begin{array}{c}0.064 \\
(0.077)\end{array}$ & $\begin{array}{l}-0.001 \\
(0.077)\end{array}$ & $\begin{array}{c}0.009 \\
(0.012)\end{array}$ & $\begin{array}{c}0.011 \\
(0.009)\end{array}$ & $\begin{array}{l}-0.021 \\
(0.076)\end{array}$ & 73,167 \\
\hline & $\begin{array}{l}\text { Add controls for } \\
\text { family income }\end{array}$ & $\begin{array}{c}0.064 \\
(0.004)\end{array}$ & $\begin{array}{c}0.043 \\
(0.111)\end{array}$ & $\begin{array}{l}-0.021 \\
(0.111)\end{array}$ & $\begin{array}{l}-0.005 \\
(0.016)\end{array}$ & $\begin{array}{c}0.037 \\
(0.023)\end{array}$ & $\begin{array}{l}-0.053 \\
(0.110)\end{array}$ & 55,908 \\
\hline 4. & Females sample & $\begin{array}{c}0.074 \\
(0.005)\end{array}$ & $\begin{array}{c}0.074 \\
(0.110)\end{array}$ & $\begin{array}{c}0.000 \\
(0.110)\end{array}$ & $\begin{array}{l}-0.004 \\
(0.012)\end{array}$ & $\begin{array}{l}-0.005 \\
(0.011)\end{array}$ & $\begin{array}{c}0.008 \\
(0.106)\end{array}$ & 59,089 \\
\hline & $\mathrm{Mal}$ & $\begin{array}{c}0.057 \\
(0.004)\end{array}$ & $\begin{array}{c}0.034 \\
(0.106)\end{array}$ & $\begin{array}{l}-0.023 \\
(0.106)\end{array}$ & $\begin{array}{c}0.038 \\
(0.029)\end{array}$ & $\begin{array}{c}0.037 \\
(0.029)\end{array}$ & $\begin{array}{l}-0.098 \\
(0.121)\end{array}$ & 60,512 \\
\hline & panic & $\begin{array}{c}0.067 \\
(0.004)\end{array}$ & $\begin{array}{c}0.077 \\
(0.108)\end{array}$ & $\begin{array}{l}0.010 \\
(0.108)\end{array}$ & $\begin{array}{l}-0.005 \\
(0.014)\end{array}$ & $\begin{array}{l}-0.007 \\
(0.014)\end{array}$ & $\begin{array}{c}0.022 \\
(0.106)\end{array}$ & 53,808 \\
\hline 7. & $\begin{array}{l}\text { Non-black } \\
\text { and non-Hispanic }\end{array}$ & $\begin{array}{c}0.064 \\
(0.004)\end{array}$ & $\begin{array}{c}0.058 \\
(0.073)\end{array}$ & $\begin{array}{l}-0.007 \\
(0.073)\end{array}$ & $\begin{array}{c}0.008 \\
(0.011)\end{array}$ & $\begin{array}{c}0.015 \\
(0.009)\end{array}$ & $\begin{array}{l}-0.029 \\
(0.073)\end{array}$ & 65,793 \\
\hline & $\begin{array}{l}\text { Direct cost } \\
\text { instruments only }\end{array}$ & $\begin{array}{c}0.065 \\
(0.003)\end{array}$ & $\begin{array}{c}0.008 \\
(0.133)\end{array}$ & $\begin{array}{l}-0.057 \\
(0.133)\end{array}$ & $\begin{array}{c}0.012 \\
(0.013)\end{array}$ & $\begin{array}{c}0.013 \\
(0.013)\end{array}$ & $\begin{array}{l}-0.083 \\
(0.130)\end{array}$ & 119,601 \\
\hline & $\begin{array}{l}\text { Opportunity cost } \\
\text { instruments only }\end{array}$ & $\begin{array}{c}0.065 \\
(0.003)\end{array}$ & $\begin{array}{c}0.131 \\
(0.113)\end{array}$ & $\begin{array}{c}0.066 \\
(0.113)\end{array}$ & $\begin{array}{c}0.009 \\
(0.020)\end{array}$ & $\begin{array}{c}0.016 \\
(0.033)\end{array}$ & $\begin{array}{c}0.033 \\
(0.109)\end{array}$ & 119,601 \\
\hline
\end{tabular}
Notes: Standard errors (in parentheses) are robust to heteroskedasticity and correlation across observations on persons living in the same county at age 14. Section D.4 describes the empirical specification for estimating the decomposition.

${ }^{a}$ Excludes oversamples of minority or economically disadvantaged individuals.

b Adds family income percentile to covariates, allowing different slopes across tertiles. Excludes persons with no observation of family income until age 17. 1957-60 birth cohorts are entirely excluded as a result.

${ }^{c}$ Uses college proximity and tuition rate as instruments.

${ }^{\mathrm{d}}$ Uses local earnings and unemployment rate at age 17 as instruments. 


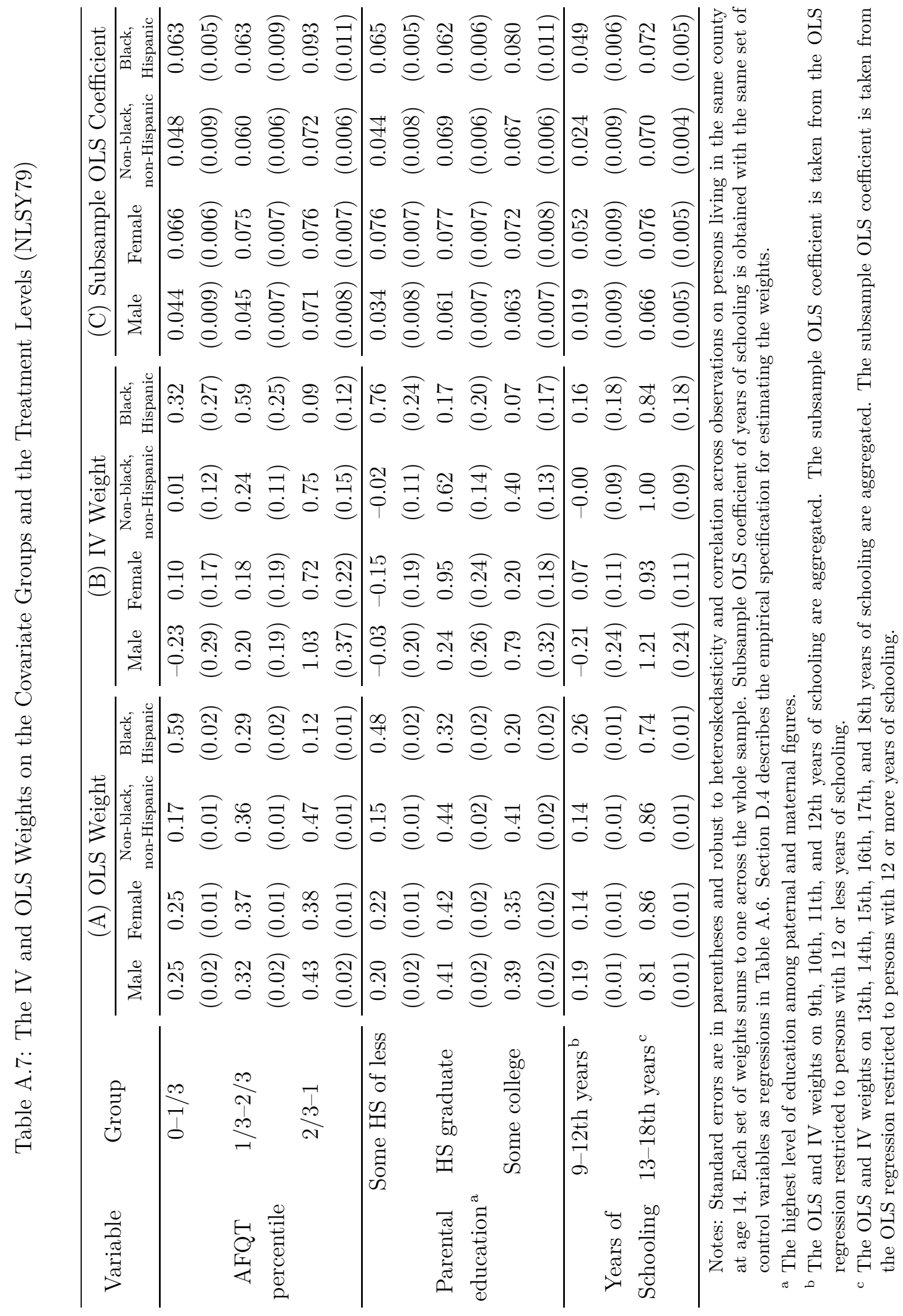


Table A.8: Descriptive Statistics (U.K. General Household Surveys 1984-98)

\begin{tabular}{lcc}
\hline & Britain & $\begin{array}{c}\text { Northern } \\
\text { Ireland }\end{array}$ \\
\hline Annual earnings $(£ 1,000)$ & 11.01 & 8.89 \\
Years of schooling & $(12.10)$ & $(6.57)$ \\
& 11.38 & 11.22 \\
Age & $(2.70)$ & $(2.61)$ \\
& 48.47 & 48.70 \\
Sample size & $(7.40)$ & $(7.36)$ \\
\hline
\end{tabular}

Notes: Standard deviations are in parentheses.

Table A.9: Alternative Specifications for Decomposing the IV-OLS Coefficient Gap (British and Northern Irish GHS)

\begin{tabular}{|c|c|c|c|c|c|c|c|}
\hline & \multicolumn{3}{|c|}{ Coefficients } & \multicolumn{3}{|c|}{ Decomposition } \\
\hline & & OLS & IV & IV-OLS & $\Delta_{C W}$ & $\Delta_{T W}$ & $\Delta_{M E}$ \\
\hline 1. & British sample & 0.084 & $\overline{0.062}$ & -0.021 & -0.016 & $\begin{array}{l}-0.029 \\
\end{array}$ & 0.023 \\
\hline & (bas & $(0.002)$ & $(0.083)$ & $(0.082)$ & $(0.009)$ & $(0.018)$ & $(0.078)$ \\
\hline 2. & British sample & 0.064 & 0.062 & -0.002 & -0.005 & -0.023 & 0.026 \\
\hline & witho & $(0.001)$ & $(0.083)$ & $(0.083)$ & $(0.008)$ & $(0.019)$ & $(0.078)$ \\
\hline 3 & Northern Ireland sample & 0.114 & 0.224 & 0.109 & -0.003 & -0.023 & 0.135 \\
\hline & & $(0.003)$ & $(0.144)$ & $(0.144)$ & $(0.005)$ & $(0.019)$ & $(0.146)$ \\
\hline & $\begin{array}{l}\text { Northern Ireland sample } \\
\text { without top-coding }\end{array}$ & $\begin{array}{c}0.105 \\
(0.004)\end{array}$ & $\begin{array}{c}0.209 \\
(0.142)\end{array}$ & $\begin{array}{c}0.104 \\
(0.142)\end{array}$ & $\begin{array}{l}-0.002 \\
(0.006)\end{array}$ & $\begin{array}{l}-0.013 \\
(0.019)\end{array}$ & $\begin{array}{c}0.119 \\
(0.139)\end{array}$ \\
\hline
\end{tabular}

Notes: Standard errors (in parentheses) are robust to heteroskedasticity and correlation across observations on the same birth cohort and survey year. For the British sample, the instrument is an indicator for turning age 14 in 1947 or later. For the Northern Ireland sample, the instrument is an indicator for turning age 14 in 1957 or later. Control variables are quartic of ages and quartic of birth cohorts. Section D.4 describes the empirical specification for estimating the decomposition. 
Table A.10: The Total IV and OLS Weights on the Covariate Groups (Northern Irish GHS)

\begin{tabular}{ccccc}
\hline Year at 14 & $\begin{array}{c}\text { Population } \\
\text { share }\end{array}$ & $\begin{array}{c}\text { OLS } \\
\text { weights }\end{array}$ & $\begin{array}{c}\text { IV } \\
\text { weights }\end{array}$ & $\begin{array}{c}\text { Subsample } \\
\text { OLS }\end{array}$ \\
\hline $1935-40$ & 0.03 & 0.03 & 0.05 & 0.064 \\
& & $(0.01)$ & $(0.04)$ & $(0.024)$ \\
$1941-45$ & 0.10 & 0.09 & -0.05 & 0.111 \\
& & $(0.02)$ & $(0.08)$ & $(0.011)$ \\
$1946-50$ & 0.15 & 0.13 & 0.01 & 0.104 \\
& & $(0.02)$ & $(0.07)$ & $(0.007)$ \\
$1951-55$ & 0.19 & 0.18 & 0.33 & 0.119 \\
& & $(0.03)$ & $(0.12)$ & $(0.008)$ \\
$1956-60$ & 0.24 & 0.26 & 0.63 & 0.116 \\
& & $(0.04)$ & $(0.13)$ & $(0.007)$ \\
$1961-65$ & 0.29 & 0.30 & 0.03 & 0.121 \\
& \multirow{2}{*}{0.29} & $(0.04)$ & $(0.08)$ & $(0.006)$ \\
\hline
\end{tabular}

Notes: Standard errors (in parentheses) are robust to heteroskedasticity and correlation across observations on the same birth cohort and survey year. Each set of weights sums to one across the whole sample. Subsample OLS coefficient of years of schooling is obtained with controlling for quartic terms of ages and birth cohorts. Section D.4 describes the empirical specification for estimating the weights.

Table A.11: Descriptive Statistics (1960-80 Censuses)

\begin{tabular}{lccc}
\hline Census year & 1960 & 1970 & 1980 \\
\hline Weekly earnings $(\$)$ & 120.9 & 207.9 & 437.5 \\
& $(67.7)$ & $(126.3)$ & $(253.1)$ \\
Years of schooling & 10.55 & 11.65 & 12.71 \\
& $(3.28)$ & $(3.27)$ & $(3.13)$ \\
Age & 44.30 & 44.48 & 44.41 \\
& $(2.85)$ & $(2.86)$ & $(2.91)$
\end{tabular}

Years of schooling required by child labor laws at 14

\begin{tabular}{lccc}
6 years or less & 0.228 & 0.195 & 0.057 \\
7 years & 0.368 & 0.242 & 0.237 \\
8 years & 0.349 & 0.496 & 0.419 \\
9 years or more & 0.054 & 0.066 & 0.286 \\
\hline Sample size & 72,494 & 161,435 & 378,177 \\
\hline
\end{tabular}

Notes: Standard deviations are in parentheses, except for indicator variables. 
Table A.12: First-Stage Coefficients of the CSL instruments

\begin{tabular}{ccc}
\hline & Coef. & \multicolumn{1}{c}{ S.E. } \\
\hline Schooling required by child labor laws & \\
7 years & 0.106 & $(0.031)$ \\
8 years & 0.107 & $(0.028)$ \\
9 years or more & 0.263 & $(0.038)$ \\
\hline Effective F-stat. & \multicolumn{2}{c}{14.88} \\
\hline
\end{tabular}

Notes: Standard errors (in parentheses) are robust to heteroskedasticity and correlation across observations on the same state and year of birth. The dependent variable is years of schooling. The control variables are state of birth and year of birth dummies. The effective F-statistics is based on Montiel Olea and Pflueger (2013).

Table A.13: Alternative Specifications for Decomposing the IV-OLS Coefficient Gap (U.S. Census)

\begin{tabular}{|c|c|c|c|c|c|c|}
\hline \multirow[b]{2}{*}{ Instruments } & \multicolumn{3}{|c|}{ Coefficients } & \multicolumn{3}{|c|}{ Decomposition } \\
\hline & OLS & $\overline{\mathrm{IV}}$ & IV-OLS & $\Delta_{C W}$ & $\Delta_{T W}$ & $\Delta_{M E}$ \\
\hline 1. $\quad$ Child labor laws & 0.067 & 0.084 & 0.017 & 0.011 & 0.003 & 0.003 \\
\hline (base specification) & $(0.0004)$ & $(0.022)$ & $(0.022)$ & $(0.004)$ & $(0.003)$ & $(0.021)$ \\
\hline 2. Compulsory & 0.067 & 0.161 & 0.094 & 0.007 & 0.004 & 0.084 \\
\hline $\begin{array}{l}\text { 3. Required schooling } \\
\text { (Stephens and Yan }\end{array}$ & 0.067 & 0.104 & 0.037 & 0.005 & 0.006 & 0.026 \\
\hline 4. Quarter of birth & $\begin{array}{c}0.067 \\
(0.0004)\end{array}$ & $\begin{array}{c}0.064 \\
(0.014)\end{array}$ & $\begin{array}{r}-0.003 \\
(0.014)\end{array}$ & $\begin{array}{c}0.000 \\
(0.0004)\end{array}$ & $\begin{array}{l}-0.002 \\
(0.001)\end{array}$ & $\begin{array}{l}-0.002 \\
(0.014)\end{array}$ \\
\hline
\end{tabular}

Notes: Standard errors (in parentheses) are robust to heteroskedasticity and correlation across observations on the same state and year of birth. The dependent variable is log weekly earnings. The control variables are state of birth and year of birth dummies. Section D.4 describes the empirical specification for estimating the decomposition. 
Table A.14: The IV and OLS Weights on the Covariate Groups with Alternative Instruments (U.S. Census)

\begin{tabular}{|c|c|c|c|c|c|c|c|}
\hline Variable & Group & $\begin{array}{l}\text { Population } \\
\text { share }\end{array}$ & $\begin{array}{c}\text { OLS } \\
\text { weights }\end{array}$ & $\begin{array}{l}\text { CA IV } \\
\text { weights }\end{array}$ & $\begin{array}{l}\text { RS IV } \\
\text { weights }\end{array}$ & $\begin{array}{l}\text { QOB IV } \\
\text { weights }\end{array}$ & $\begin{array}{c}\text { Subsample } \\
\text { OLS }\end{array}$ \\
\hline \multirow{3}{*}{$\begin{array}{l}\text { Year of } \\
\text { birth }\end{array}$} & $1910-19$ & 0.32 & $\begin{array}{c}0.33 \\
(0.02)\end{array}$ & $\begin{array}{c}1.31 \\
(0.21)\end{array}$ & $\begin{array}{c}1.65 \\
(0.18)\end{array}$ & $\begin{array}{c}0.45 \\
(0.05)\end{array}$ & $\begin{array}{c}0.063 \\
(0.001)\end{array}$ \\
\hline & $1920-29$ & 0.35 & $\begin{array}{c}0.36 \\
(0.02)\end{array}$ & $\begin{array}{c}1.71 \\
(0.24)\end{array}$ & $\begin{array}{c}0.84 \\
(0.17)\end{array}$ & $\begin{array}{c}0.27 \\
(0.04)\end{array}$ & $\begin{array}{c}0.070 \\
(0.001)\end{array}$ \\
\hline & 1930-39 & 0.33 & $\begin{array}{c}0.31 \\
(0.02)\end{array}$ & $\begin{array}{l}-2.01 \\
(0.33)\end{array}$ & $\begin{array}{l}-1.50 \\
(0.20)\end{array}$ & $\begin{array}{c}0.28 \\
(0.03)\end{array}$ & $\begin{array}{c}0.067 \\
(0.001)\end{array}$ \\
\hline \multirow{4}{*}{$\begin{array}{c}\text { Region of } \\
\text { birth }\end{array}$} & Northeast & 0.29 & $\begin{array}{c}0.26 \\
(0.02)\end{array}$ & $\begin{array}{l}-0.46 \\
(0.46)\end{array}$ & $\begin{array}{c}0.54 \\
(0.20)\end{array}$ & $\begin{array}{c}0.10 \\
(0.04)\end{array}$ & $\begin{array}{c}0.069 \\
(0.001)\end{array}$ \\
\hline & & 0.33 & $\begin{array}{c}0.27 \\
(0.02)\end{array}$ & $\begin{array}{l}-1.17 \\
(0.34)\end{array}$ & $\begin{array}{l}-1.43 \\
(0.21)\end{array}$ & $\begin{array}{c}0.20 \\
(0.04)\end{array}$ & $\begin{array}{c}0.066 \\
(0.001)\end{array}$ \\
\hline & South & 0.30 & $\begin{array}{c}0.39 \\
(0.02)\end{array}$ & $\begin{array}{c}3.21 \\
(0.41)\end{array}$ & $\begin{array}{c}2.69 \\
(0.26)\end{array}$ & $\begin{array}{c}0.63 \\
(0.05)\end{array}$ & $\begin{array}{c}0.068 \\
(0.001)\end{array}$ \\
\hline & West & 0.09 & $\begin{array}{c}0.08 \\
(0.01)\end{array}$ & $\begin{array}{l}-0.57 \\
(0.11)\end{array}$ & $\begin{array}{l}-0.80 \\
(0.10)\end{array}$ & $\begin{array}{c}0.06 \\
(0.02)\end{array}$ & $\begin{array}{c}0.064 \\
(0.001)\end{array}$ \\
\hline
\end{tabular}

Notes: Standard errors (in parentheses) are robust to heteroskedasticity and correlation across observations on the same state and year of birth. Each set of weights sums to one across the whole sample. Subsample OLS coefficient of years of schooling is obtained with the same set of control variables as regressions in Table A.13 "CA IV" refers to compulsory attendance instruments, "RS IV" refers to required schooling instruments as defined by Stephens and Yang (2014), and "QOB IV" refers to quarter-of-birth instruments. Section D.4 describes the empirical specification for estimating the weights. 
Figure A.1: The IV and OLS Weights on the Treatment Levels (Northern Irish GHS)

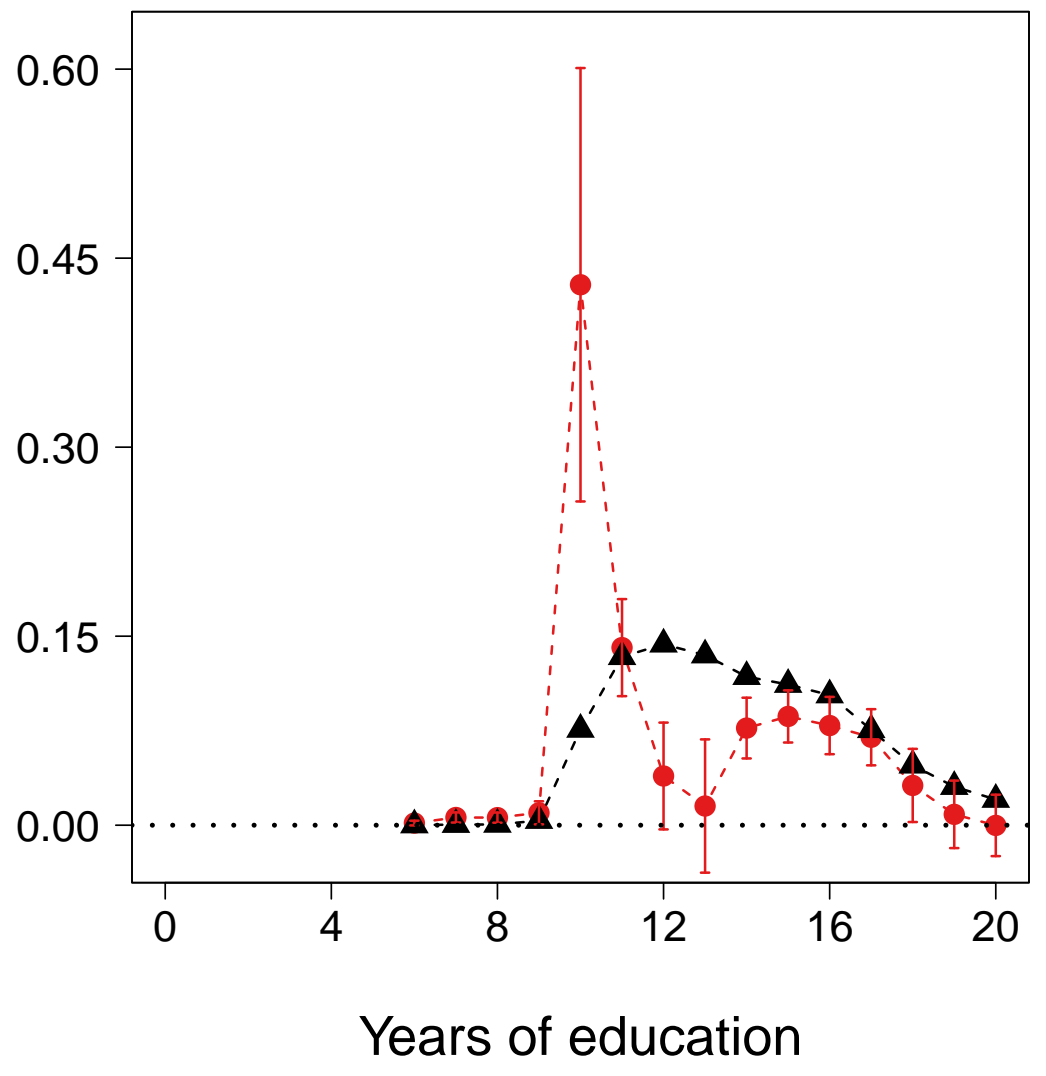

- - IV weight - - - OLS weight

Notes: The IV weights are presented with standard error bars. The standard error bars for the OLS weights are omitted because they are graphically negligible. Each set of weights sums to one across the whole sample. Section D.4 describes the empirical specification for estimating the weights. 
Figure A.2: The IV and OLS Weights on the Treatment Levels with Alternative Instruments (U.S. Census)

(a) Compulsory Attendance Laws

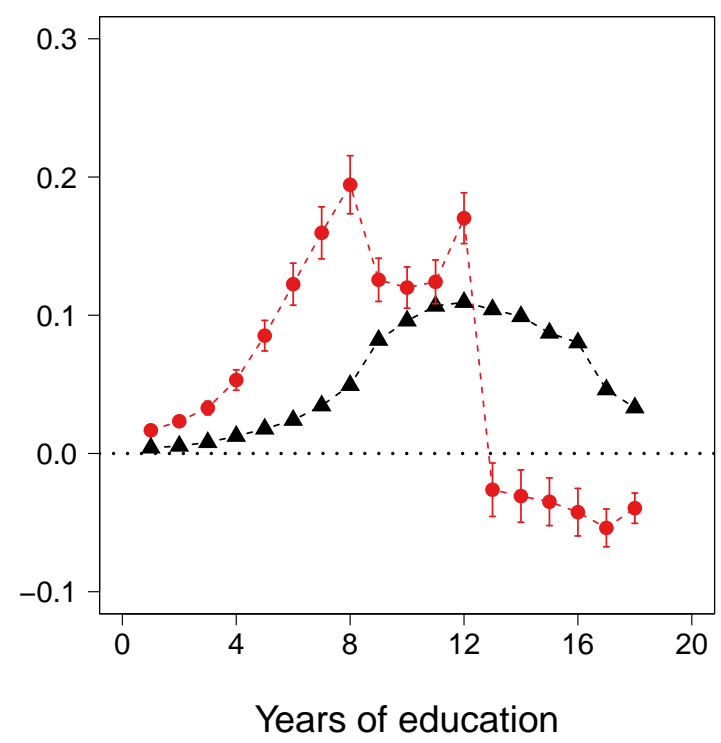

(c) Quarter of Birth

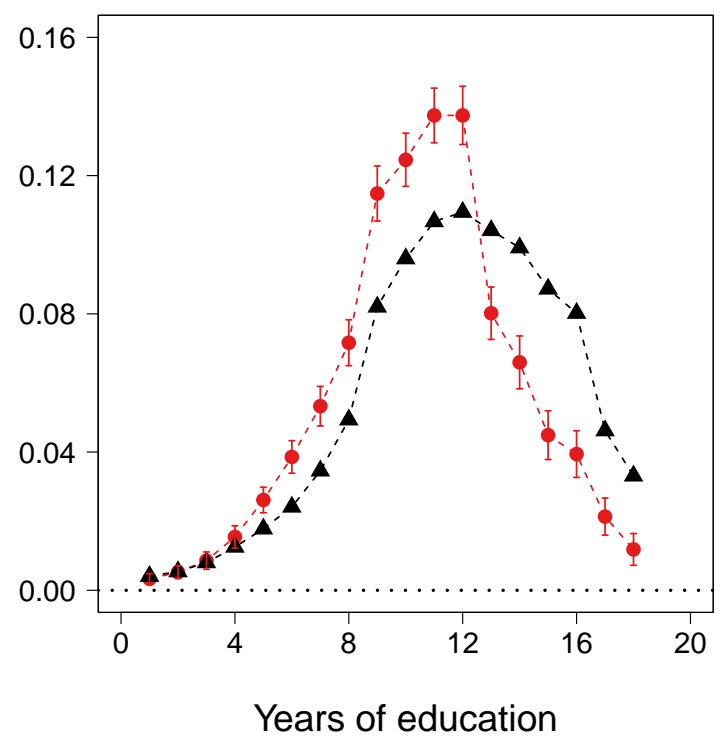

(b) Required Schooling

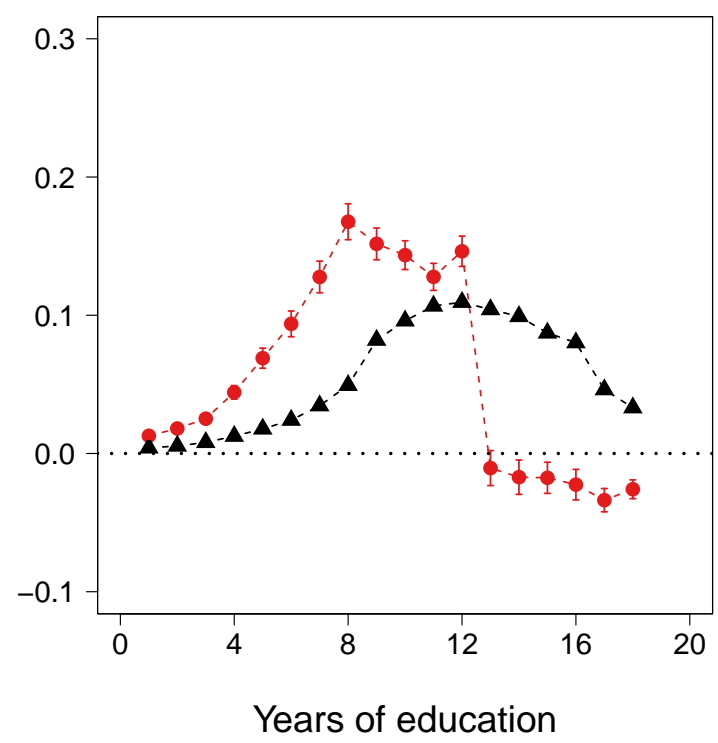

-- IV weight - - - OLS weight

Notes: The IV weights are presented with standard error bars. The standard error bars for the OLS weights are omitted because they are graphically negligible. Each set of weights sums to one across the whole sample. Section D.4 describes the empirical specification for estimating the weights. 